\title{
Optimal Thresholding of Predictors in Mineral Prospectivity Analysis
}

\author{
Adrian Baddeley • Warick Brown • Robin K. Milne • \\ Gopalan Nair • Suman Rakshit • Tom Lawrence • \\ Aloke Phatak • Shih Ching Fu
}

\section{A. Baddeley}

School of Electrical Engineering, Computing and Mathematical Sciences,

Curtin University, GPO Box U1987, Perth WA 6845, Australia

E-mail: adrian.baddeley@curtin.edu.au

W. Brown

John de Laeter Centre, Curtin University

R. K. Milne

Department of Mathematics and Statistics, University of Western Australia

G. Nair

Department of Mathematics and Statistics, University of Western Australia

S. Rakshit

SAGI-West, School of Molecular and Life Sciences, Curtin University

\section{A. Phatak}

Centre for Transforming Maintenance through Data Science, Curtin University

T. Lawrence

S. C. Fu

Curtin University

ORCID : 0000-0001-9499-8382

ORCID : 0000-0002-8257-888X

ORCID : 0000-0002-1098-2672

ORCID : 0000-0003-3883-4986

ORCID : 0000-0003-0052-128X

ORCID : 0000-0002-0637-7461

ORCID : 0000-0003-3648-7634

ORCID : 0000-0002-9077-6026 


\section{INTRODUCTION}

In many techniques for mineral prospectivity analysis, especially Weights of Evidence (WofE), the predictor variables are required to be binary (0 or 1) values (Agterberg 1992; Agterberg et al. 1993; Bonham-Carter et al. 1990). Geological survey data of other kinds can be used, provided they are first converted to binary values by thresholding (Goodacre et al. 1993). If the original evidence layer is a geochemical or geophysical variable, such as an isotope abundance ratio, magnetic field anomaly or gravitational field strength, the analyst chooses a threshold above which the value is labelled as anomalous. If the original evidence is a spatial feature, such as a fault system, the analyst will often choose a cutoff distance to define a buffer around the feature. We shall use the term 'threshold' in either case.

The crucial step is to choose the best value of the threshold. This can be quite difficult in practice (J. Liu and Cheng 2019, p. 1039). A poor choice of threshold can be costly, leading to poor prediction, wasted exploration effort, and failure to identify important predictor variables.

Several methods for selecting the threshold are commonly used in prospectivity analysis. They include choosing the threshold value which maximises the WofE contrast (Goodacre et al. 1993, pp. 291-292; Bonham-Carter 1995, pp. 321-322), the Studentised contrast (Bonham-Carter 1995, p. 325), the chi-squared test statistic (BonhamCarter and Agterberg 1990), the Youden criterion (Chen and Wu 2019; Ruopp et al. 2008), or the curvature of the capture-efficiency curve (Porwal et al. 2010). The prospectivity literature contains only limited discussion about the relative performance of these methods (Ford et al. 2016; Yeomans 2018, Chapter 7) and there does not seem to be a consensus about the best method. There are also some concerns that, for example, the Studentised contrast may be unreliable when the number of deposits is small (Goodacre et al. 1993, pp. 291, 295).

Threshold selection is an instance of change-point estimation, a classical and widely-studied problem in statistical theory (Basseville and Nikiforov 1993; Chernoff and Rubin 1956; Page 1957; Pons 2018; Zacks 1983). The statistical literature on this problem suggests that the WofE contrast would perform poorly as a criterion for threshold selection, because the contrast is an estimate of effect size rather than of evidence for the threshold. The change-point literature offers some other promising methods for threshold selection, including cumulative sum (CUSUM) methods (Basseville and Nikiforov 1993, Chapter 2), profile likelihood (Galun and Trifonov 1982; Murphy and van der Vaart 2000; Kutoyants 1998, Chapter 5, pp. 183-224) and the method of Akman and Raftery (1986). The existence of so many different competing methods is unusual; statistical theory usually identifies a single method as being "optimal", but the optimality theory is not applicable in this context, because prospectivity is not a differentiable function of the predictor. Furthermore, in prospectivity analysis the number of known deposits may be quite small, so that asymptotic statistical theory does not apply. Consequently, these alternative methods are not guaranteed to have better practical performance than the currently popular methods listed above.

In this paper we give an overview of all the methods of threshold selection listed above, and evaluate their performance on real and simulated data. We go into detail about each method, identify connections between them, and draw attention to common technical errors. We present graphical diagnostics which may reveal numerical quirks of the data and assist the analyst to reject geologically implausible results. We highlight the central role of the capture-efficiency curve and offer new insights about it. We also show how to calculate standard errors and confidence intervals for the threshold using bootstrap techniques.

Our main findings are as follows. The method of threshold selection based on maximising the WofE contrast is highly unreliable and should be avoided. Threshold selection using the Studentised contrast $t$ has adequate performance. The Youden criterion stands out as having the best performance. These findings assume that a simple binary threshold relationship truly holds (which can be checked by inspecting the shape of the captureefficiency curve). If the relationship between predictor and prospectivity is more complicated, the maximum profile likelihood method is the most suitable.

Despite their very different origins, we find that all the methods mentioned above are mathematically related, so they should not be regarded as independent sources of evidence; in particular, the Akman-Raftery and chi-squared statistics are mathematically equivalent, and the Youden criterion is equivalent to a CUSUM index.

Typically, the survey data will comprise many predictors (evidence layers). In the WofE approach, each variable is examined separately to determine its merit and suitability as a predictor, and to select the single best threshold value for that variable. This is different from other approaches such as CART, random forest and isolation forest methods (Breiman et al. 1984; Chen and Wu 2019) which use a decision tree of threshold rules that are optimized by considering all the predictors at once. The task of selecting correct thresholds is even more challenging in these multivariate approaches. For simplicity, and because WofE is so popular, we shall broadly follow the WofE approach and consider only one predictor at a time. However, our conclusions are also relevant to other techniques for mineral prospectivity analysis. 
When many predictor variables are available, the geologist must decide which of the variables have an effect on prospectivity. Statistical tests of significance are often used. There are connections between significance tests and the criteria employed to select the threshold (Bonham-Carter 1995; Bonham-Carter and Agterberg 1990). The Youden criterion, which we found to have the best performance for selecting thresholds, is closely related to the Kolmogorov-Smirnov test (Conover 1999, pp. 295-301) which is known to have good performance and is calibrated for any sample size (i.e. for any number of deposits). Consequently, we recommend using the Kolmogorov-Smirnov test for significance testing.

The paper is organised as follows. First we briefly review terms and notation for binary predictors. Then we define the problem of choosing a threshold value for a numerical ("greyscale") predictor, define notation, and introduce two example datasets. The next five sections describe different classes of methods in detail: those based on the estimated WofE contrast and Studentised contrast; one based on the $\chi^{2}$ test statistic; ones based on the captureefficiency curve; and methods borrowed from the change-point literature. The next section exposes mathematical connections between the different methods. Then we discuss statistical significance tests. We report on a simulation experiment and on analysis of data from the Murchison goldfields, Yilgarn craton, Western Australia. A further section describes bootstrap methods for calculating standard errors and confidence intervals for the threshold. The final section discusses further aspects of performance, model mis-specification and generalisations. Appendices provide mathematical details.

\section{BINARY PREDICTORS}

Thresholding converts numerical ("greyscale") predictor values into binary predictor values, for use in prospectivity analysis techniques that require binary inputs. This paper is concerned only with the thresholding step, and not with the prospectivity analysis.

However, many threshold selection techniques involve prospectivity analysis: they consider every possible threshold value in turn, perform a prospectivity analysis using that threshold, and select the threshold value which gives the best result. In order to describe these techniques, we must first define terms and notation for prospectivity using a binary predictor.

The general scenario is sketched in Figure 1. There is a survey region $S$ in two-dimensional space, within which we have identified an areal feature or subregion $B$ that is believed to be prospective. The binary predictor takes the value 1 inside $B$, and the value 0 outside $B$. The spatial pattern of known locations of mineral deposits is also mapped.

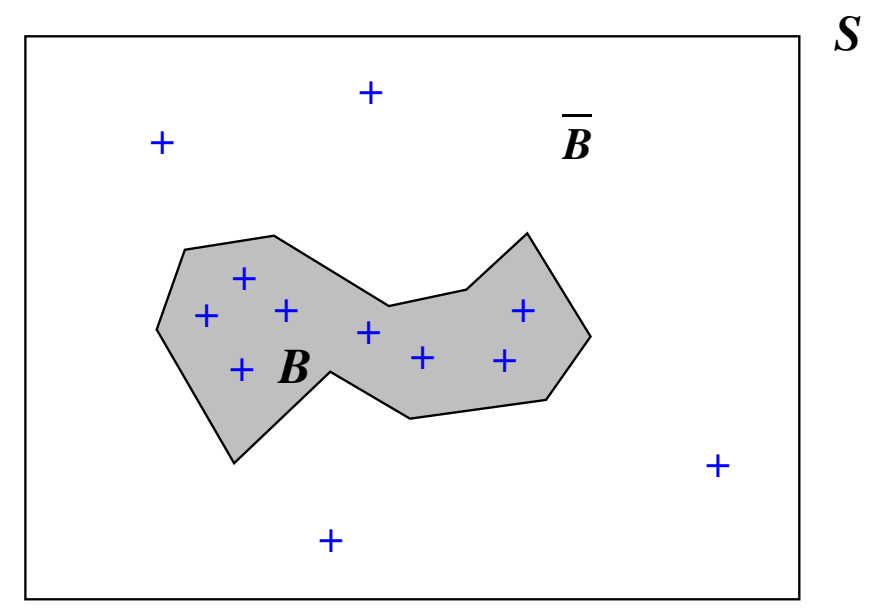

Fig. 1 Sketch of setup for prospectivity analysis once a threshold has been selected. The survey region $S$ (rectangular frame) contains an areal feature $B$ (shaded), believed to be prospective for deposits (blue crosses). The feature $B$ has area $a_{B}$ and contains $n_{B}$ deposits. The complementary region $\bar{B}$ (the unshaded part of the survey region outside the prospective feature) has area $a_{\bar{B}}$ and contains $n_{\bar{B}}$ deposits. 
For quantitative analysis of prospectivity, the survey region is divided into grid cells of equal area. To simplify the discussion and the equations, we assume throughout this paper that grid cell area is negligible ${ }^{1}$ compared to the area of the study region. The appendix Non-negligible Grid Cell Size gives the more complicated formulae that apply when grid cell area cannot be ignored.

In each grid cell, the survey records the value of the predictor, and records whether or not there are any (known) mineral deposits in the cell. Overall there are $n$ grid cells that contain deposit locations; amongst them are $n_{B}$ grid cells containing deposits lying inside feature $B$, and $n_{\bar{B}}$ grid cells containing deposits lying outside $B$. The total area of all grid cells, the area of the domain $S$, is denoted by $a$; the area of $B$ is denoted $a_{B}$ and the area of its complement is $a_{\bar{B}}$.

The data listed above are enough to perform prospectivity analysis using the binary predictor. The most familiar of these methods is WofE (Agterberg 1992; Agterberg and Bonham-Carter 1999; Agterberg et al. 1993; Agterberg and Cheng 2002; Carranza 2004; Bonham-Carter 1995, Chapter 9; Agterberg 2014, Chapter 5). Under our assumption that cell areas are negligible, the estimated weights of evidence are $W_{+}=\ln \left(n_{B} / a_{B}\right)-\ln (n / a)$ for cells inside $B$, and $W_{-}=\ln \left(n_{\bar{B}} / a_{\bar{B}}\right)-\ln (n / a)$ for cells outside $B$, where ln denotes the natural logarithm. The WofE contrast is

$$
\widehat{C}=W_{+}-W_{-}=\ln \frac{n_{B}}{a_{B}}-\ln \frac{n_{\bar{B}}}{a_{\bar{B}}}=\ln \left(\frac{n_{B} / a_{B}}{n_{\bar{B}} / a_{\bar{B}}}\right)=\ln \left(\frac{n_{B} / n_{\bar{B}}}{a_{B} / a_{\bar{B}}}\right) .
$$

(For non-negligible cell area, the corresponding formula is given in the appendix Non-negligible Grid Cell Size as equation (41).) It is instructive to notice that $n_{B} / a_{B}$ is the observed average density of mineral deposit locations (deposits per unit area) inside the prospective region $B$, while $n_{\bar{B}} / a_{\bar{B}}$ is the observed average density of deposits outside $B$. The calculated contrast $\widehat{C}$ is the logarithm of the ratio of these two observed densities, or equivalently, it is the difference between the logarithms of the two observed densities. A calculated contrast value of $\widehat{C}=+1$ means that the observed density of mineral deposit locations inside the prospective region $B$ exceeds the density outside by a multiplicative factor of $e^{+1}=2.7$. A calculated contrast value of $\widehat{C}=0$ means that the observed densities of deposits inside and outside the prospective region are equal.

For a single binary predictor, WofE is equivalent to logistic regression (Agterberg 1974; Schaeben 2014; Schaeben and Semmler 2016), and is also equivalent to maximum Poisson likelihood estimation and to maximum entropy modelling (Baddeley et al. 2010; Baddeley 2018; Baddeley et al. 2015, Chapter 9). Equivalence means that these techniques use different formulations but yield the same predictions.

WofE has been applied to mineral prospectivity analysis for gold (Agterberg and Bonham-Carter 2005; Boleneus et al. 2001; Cassard et al. 2008; Cheng 2008; J. Harris et al. 2015; Zhang and Zhou 2015), copper (Carranza 2004; D. Harris et al. 2003; Wang et al. 2016), tungsten (Y. Liu et al. 2014; Yeomans 2018), magmatic nickel sulfide (Porwal et al. 2010) and Sn-Cu deposits (Cheng 2007). WofE has also been used to create maps of landslide susceptibility (Cervi et al. 2010; Neuhäuser and Terhorst 2007; Polykretis and Chalkias 2018) and ecological habitat quality (Gorney et al. 2011; Romero-Calcerrada et al. 2010; Romero-Calcerrada and Luque 2006).

Modifications of WofE are described in Agterberg (2011); J. Liu and Cheng (2019). Comparative reviews of the performance of WofE and other methods are given by Ford et al. (2016) and Yeomans (2018, Chapter 7).

\section{THE THRESHOLDING PROBLEM}

In this section, we define the thresholding problem, discuss what information is required to select the best threshold, and introduce some notation that will be used throughout the paper. We also describe two datasets - one real and one synthetic - that will be used to motivate and illustrate the different thresholding methods.

\section{Murchison (Western Australia) Gold Survey}

The Murchison gold survey data, shown in Figure 2, were extracted from a regional survey (scale 1:500,000) of the Murchison goldfields area by the Geological Survey of Western Australia (GSWA). The figure shows the known locations of gold deposits and occurrences, and the known or inferred locations of geological faults, in a $330 \times 400$ kilometre rectangular study region.

These data were first presented and analysed in Knox-Robinson and Groves (1997). Gold deposit and occurrence locations were obtained from a database compiled by the Geological Survey of Western Australia (Geological Survey

\footnotetext{
${ }^{1}$ While we have assumed that grid cell area is negligible in order to simplify and clarify the equations, this assumption is also pragmatically justifiable. If the survey region is $100 \mathrm{~km}$ across and grid cells are 100 metre squares, then there are a million grid cells, each with an area fraction of $10^{-6}$, and our simplified equations are accurate to 4 decimal places.
} 


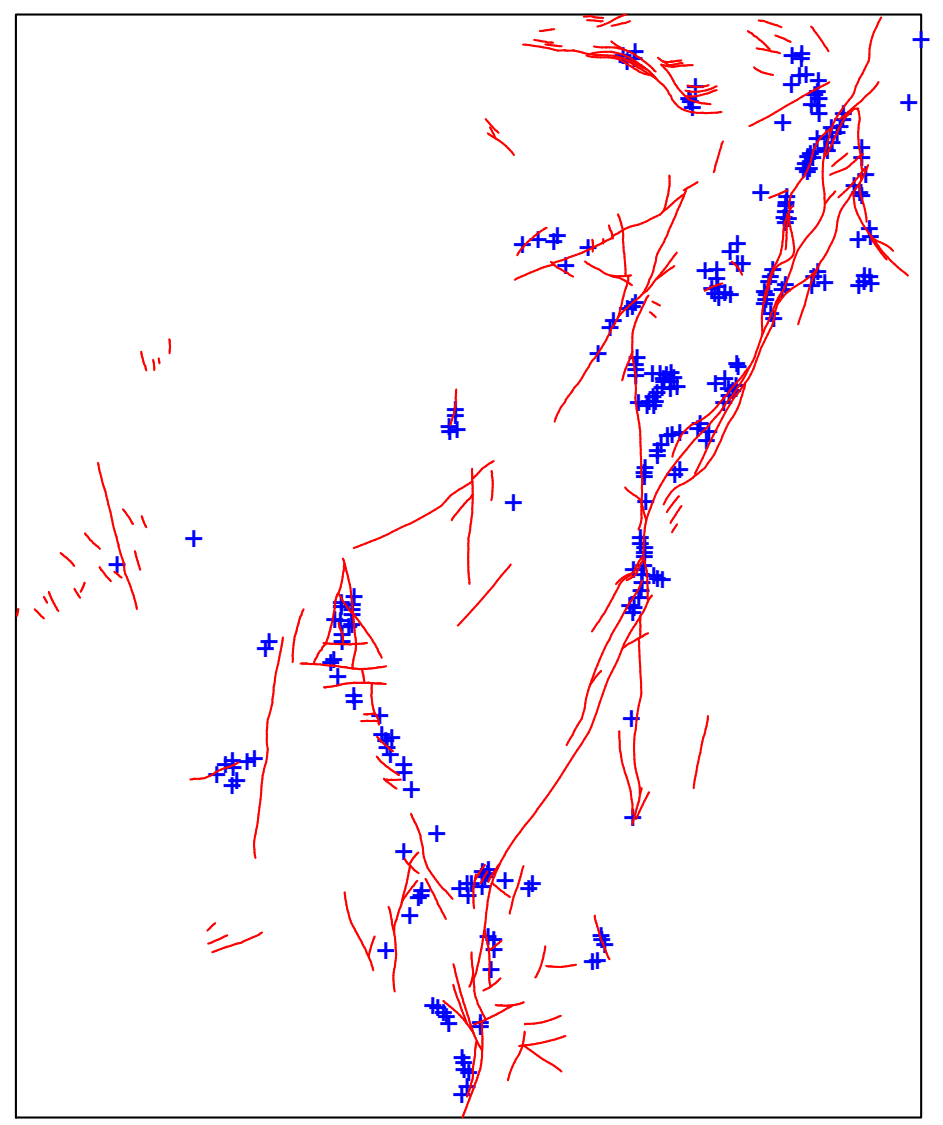

Fig. 2 Murchison gold data. Gold deposits (+) and major faults (-) in a survey region 330 by $400 \mathrm{~km}$.

of Western Australia 1994); they include deposits of all sizes and are based on map surveys at a scale of 1:50,000 or better (Knox-Robinson and Groves 1997). Fault locations were compiled by Watkins and Hickman (1990). The data were previously discussed in Groves et al. (2000), Foxall and Baddeley (2002), and Baddeley et al. (2015). Data were kindly provided by Dr Carl Knox-Robinson, and permission granted by Dr Tim Griffin, Geological Survey of Western Australia and by Dr Knox-Robinson.

Although there exist more up-to-date versions of this survey, we use the original 1994 version for demonstration purposes because this is widely available, allowing comparison between different published analyses of the same data. Users of the R software environment can obtain the data from the open-source contributed package spatstat described in Baddeley et al. (2015) and accessible via www.spatstat.org.

Figure 2 strongly suggests that proximity to faults is predictive for gold prospectivity. This is expected because crustal to lithospheric-scale faults and shear zones represent a first-order control on the location of orogenic gold deposits at a province scale. These major fluid pathways focus fluid migration along pressure gradients into dilation or damage zones in suitably-oriented, lower-order structures where gold may be deposited (Solomon and Groves 1994). Orogenic gold deposits are found world-wide and throughout geological time (Goldfarb and Groves 2015; Groves et al. 2000; Groves and Santosh 2016; Robert et al. 2005).

In order to apply mineral prospectivity techniques, we would first have to convert the faults into a binary variable, typically by creating a buffer around them. This process is discussed in the next section.

\section{Thresholding a Greyscale Predictor}

Goodacre et al. (1993) extended the WofE method to predictor variables that are real numbers ("greyscale values") instead of binary values. Examples of such predictors include isotope ratios, geochemical assay, magnetic or gravitational field strength, and distance from a fault system. The predictor is converted to a binary variable by thresholding. Threshold selection is discussed by Goodacre et al. (1993), Bonham-Carter (1995, Section 9.5, pp. 319-325), Cheng (2004), and J. Liu and Cheng (2019). 
The predictor is a spatial variable $Z$ whose values are real numbers. That is, at any spatial location $u$ in the survey region $S$, the value $Z(u)$ of the predictor is a real number that can be extracted or calculated from data. The task is to find the "best" threshold value $z$ to convert the numerical variable $Z$ to a binary variable, where the definition of "best" is open to discussion.

Figure 3 sketches this scenario. It shows a generic predictor $Z$ which takes numerical values, depicted as a contour plot; alternatively $Z$ could have been displayed as a greyscale pixel image. Thresholding effectively selects one of the contour levels, and the region below this contour level is designated as the prospective region $B$, to be interpreted as in Figure 1.

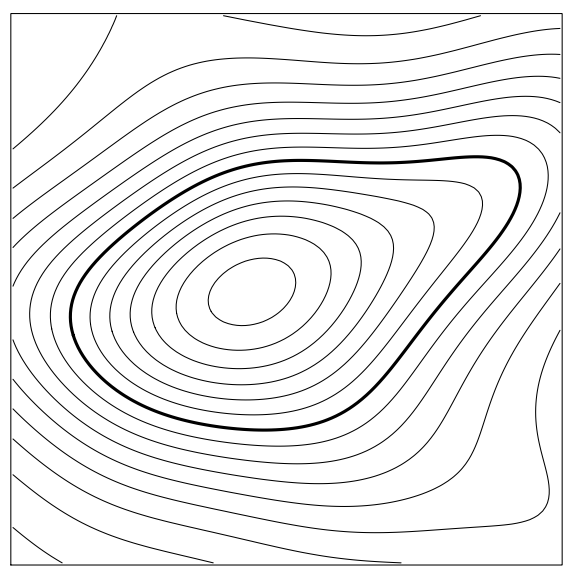

Fig. 3 Thresholding of a numerical predictor such as a geochemical or geophysical variable. Thin lines are contours of the spatial variable. One contour level (thick line) is selected as the optimal threshold.

If the original evidence layer is a geochemical variable, then Figure 3 represents contours of this variable. Thresholding has the effect of classifying each pixel as either "background" or "anomalous". The "background" is the spread of geochemical assay values expected to be seen in non-mineralised Earth materials, and the upper limit of the background range is the "threshold" (Carranza 2009, pp. 9-10). Figure 3 illustrates the selection of one contour level as the optimal threshold.

If the original evidence layer is a spatial feature such as a fault system, then the researcher may choose to define $Z(u)$ as the shortest distance from the query location $u$ to the feature. Distance values may be computed for each grid cell in a raster using the distance transform algorithm, or computed by vector geometry for any spatial location. Thresholding the distance variable at a particular threshold $z$ is equivalent to creating a buffer of distance $z$ around the original feature using GIS functionality (Cheng 2008, Figure 6). Buffers at different distances are contours of the distance function $Z(u)$. For the Murchison data in Figure 2, for example, Figure 4 shows a 5 km buffer around the major faults. Other examples of distance predictors include distance from a moraine, distance from a relatively steep slope zone, and distance from a relatively thick glacial drift layer (Cheng 2004, Table 1).

Once we have chosen a threshold level or threshold distance, the prospectivity analysis reduces to the situation sketched in Figure 1.

For simplicity in the exposition, we shall assume throughout that smaller values of $Z$ are more prospective than large values. For example, this is true for orogenic gold in the Yilgarn craton (containing the Murchison survey) when $Z$ is taken to be the distance to the nearest large fault. By adopting a particular threshold value $z$ we are specifying the region of high prospectivity $B$ as the spatial region consisting of all grid cells or locations $u$ where the value of $Z$ is at or below the threshold, $Z(u) \leq z$. The region of lower prospectivity $\bar{B}$ consists of all locations where $Z(u)>z$. In the particular case where $Z(u)$ is the distance to a fault system, the prospective region is a buffer around the fault system, delimited by the contour at distance $z$ around the faults.

Thresholding is a necessary step in some techniques for prospectivity analysis (such as WofE) but it may also be desirable for pragmatic reasons, because it leads to predictive models which are very simple to apply, and which drastically reduce the search area. The distance function $Z(u)$ could have been chosen simply because a buffer at a certain distance from the fault system is easy to delineate for exploration.

Mineral prospectivity analyses are often based on a small number of known deposits, although this number depends on the size of the study region and the inclusion criteria for the study (for example, when considering gold deposits with a resource of at least one tonne total contained gold). It is important that the technique for selecting the threshold should still work when the number of deposits is small. We should be able to state the minimum 


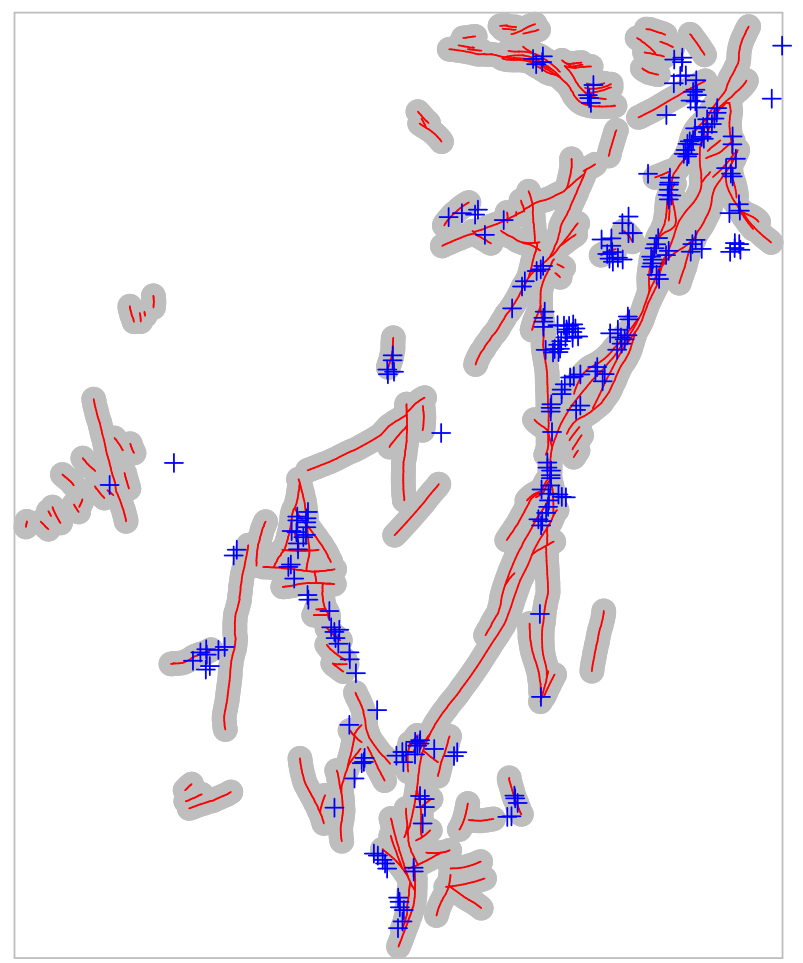

Fig. 4 Murchison gold data of Fig. 2 showing buffer at distance $5 \mathrm{~km}$ from the major faults.

number of deposits required for validity of the technique. For the thresholding methods described in this paper, the minimum required number of deposits is discussed in the following five sections (from THRESHOLD SELECTION USING WOFE CONTRAST to THRESHOLD SELECTION USING CHANGE-POINT ANALYSIS) and summarised in Table 10 of the Discussion.

\section{Synthetic Example}

We have also constructed a synthetic dataset that is superficially similar to the Murchison gold data, but has the virtue that the correct answer is known, so that the performance of threshold selection methods can be evaluated and compared.

The synthetic data, shown in Figure 5, are constructed so that there are strong contrasting densities of deposits inside and outside a $2 \mathrm{~km}$ buffer zone around the fault lines. This provides a basis for evaluating the performance of threshold selection methods, under controlled conditions. A threshold method which performs optimally in this example should select a threshold close to $2 \mathrm{~km}$ (as we discuss below). Although the synthetic dataset is not very realistic, any methods which work for complex real-world data must surely be expected to perform well on this simple dataset.

The linear features in the synthetic example are obtained from real data. A survey ${ }^{2}$ of copper deposits in Queensland, Australia comprised the spatial locations of known copper deposits and of observed lineaments which are mostly faults. We have discarded the copper deposit points and simplified the lineament pattern by randomly deleting a fraction of the lineaments. We created a buffer region, consisting of all spatial locations (grid cells) lying no further than $2 \mathrm{~km}$ from the nearest lineament, shown in the left panel of Figure 5 . We then simulated a random spatial pattern of deposits (shown in the right panel of Figure 5), with an average density of 2 deposits per $100 \mathrm{~km}^{2}$ within the buffer, and 1 deposit per $100 \mathrm{~km}^{2}$ outside the buffer. For example, in a $100 \times 100$ metre grid cell (area $0.01 \mathrm{~km}^{2}$ ) inside the buffer the expected number of deposits inside the grid cell is number $=$ density $\times$ grid cell area $=$ $(2 / 100) \times 0.01=0.0002$. Since the probability of more than one deposit is negligible, the probability that the grid

\footnotetext{
${ }^{2}$ The original survey was compiled by Dr. Jonathan Huntington, CSIRO and has been analysed by Berman (1986) and others. Coordinates were kindly provided to us by Dr. Mark Berman and Dr. Andy Green, CSIRO. The survey data are publicly available in the R package spatstat (Baddeley et al. 2015; Baddeley and Turner 2005) as the dataset copper. The data in Figure 5 are supplied in Online Resource 1.
} 

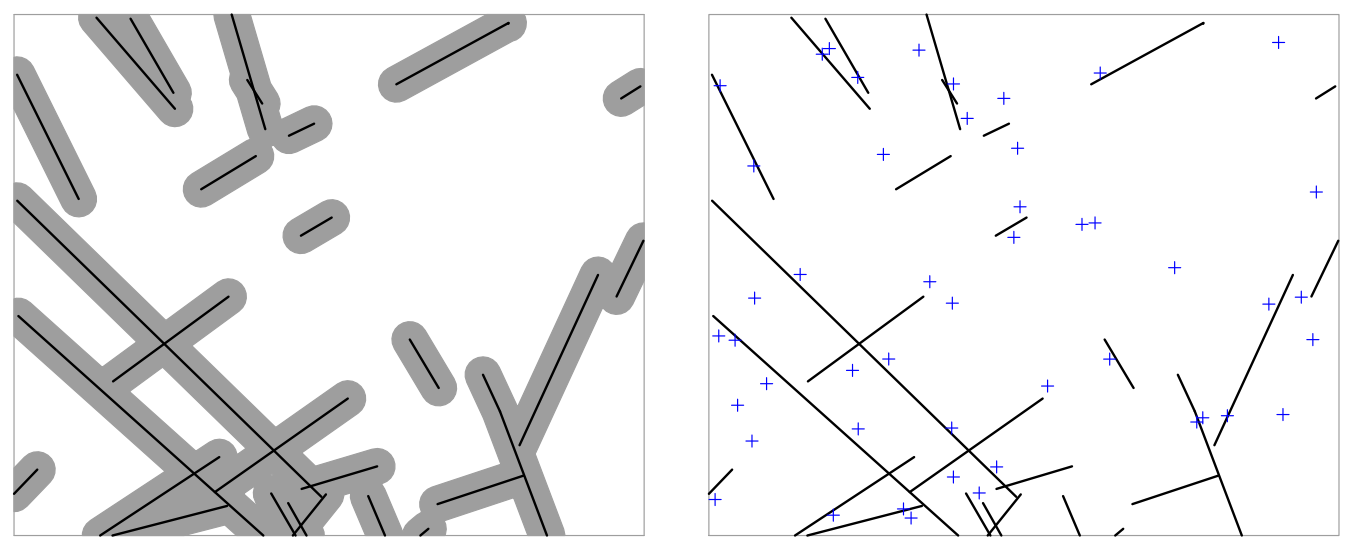

Fig. 5 Synthetic data example in a rectangle approximately 70 by $60 \mathrm{~km}$. Left: Lineaments $(-)$ and region of high prospectivity (grey shading) defined as the buffer within $2 \mathrm{~km}$ of the nearest lineament. Right: Typical simulated outcome. Mineral deposits $(+)$ generated randomly with average density 2 deposits per $100 \mathrm{~km}^{2}$ inside the high prospectivity region and 1 deposit per $100 \mathrm{~km}^{2}$ outside.

cell contains a deposit is also equal to 0.0002 . This synthetic model is typical of models that have been applied to the original dataset.

There is a single correct value of the threshold in this synthetic scenario. The true probability of a deposit depends on whether a grid cell is within or beyond the $2 \mathrm{~km}$ threshold. Therefore, a threshold of $2 \mathrm{~km}$ must be chosen in order that any prospectivity method correctly predicts the probability of a deposit. Bonham-Carter (1995, p. 319) defines the "best" threshold as "[...] the threshold that maximises the spatial association between the resulting binary map pattern and the point pattern [...]". According to this definition, the "best" threshold is also $2 \mathrm{~km}$.

The right panel of Figure 5 shows one dataset generated from this model, which we shall use to demonstrate the calculations and graphics. Since we know the correct threshold distance for this synthetic dataset is $2 \mathrm{~km}$, we count $n_{B}=34$ deposit points inside the buffer and $n_{\bar{B}}=14$ points outside. The total area of the survey region is $a=4102 \mathrm{~km}^{2}$; the area inside the buffer is $a_{B}=1294 \mathrm{~km}^{2}$, and outside the buffer $a_{\bar{B}}=4102-1294=2808 \mathrm{~km}^{2}$. The estimated contrast value calculated according to equation (1) is $\widehat{C}=\ln \frac{34 / 1294}{14 / 2808}=\ln \frac{0.026}{0.005}=1.66$. In this synthetic example, the "true" value of the contrast is the logarithm of the ratio of the "true" densities of deposit points inside and outside $B$, which is $C_{\text {true }}=\ln (0.02 / 0.01)=\ln (2)=0.69$.

\section{Essential Data Required for Threshold Selection}

For any numerical predictor, the minimum information necessary to select the best threshold is contained in the two graphs shown in Figure 6, using the synthetic data as an illustrative example. The graphs show the cumulative number of deposits (left panel) and the cumulative area (right panel) as a function of the threshold value on the horizontal axis. For any candidate threshold value $z$, let $n(z)$ be the number of grid cells with predictor value $Z \leq z$ which contain deposits, and $a(z)$ the total area of all grid cells with $Z \leq z$. (Strictly speaking the definition of $a(z)$ should exclude any grid cells which contain data points, but we have assumed grid cells are small enough to allow us to neglect this discrepancy.) The left panel of Figure 6 shows the number of deposits $n(z)$ plotted against the candidate threshold distance $z$. The right panel shows the area $a(z)$ plotted against $z$. Other transformations of these graphs will be used to select the threshold, as described in the next two sections.

The minimum data that need to be extracted from the mineral deposits are the predictor values $z_{1}, \ldots, z_{n}$ at these deposits, that is, $z_{i}=Z\left(x_{i}\right)$ for each $i$, where $x_{1}, \ldots, x_{n}$ are the deposit locations. Table 1 summarises our mathematical notation for the main elements of the problem.

\section{Overview of Methods}

In the following sections, we discuss different criteria for selecting the threshold. These criteria are summarised in Table 2 and the equations defining them are listed. The notation for the Akman-Raftery and Youden criteria 

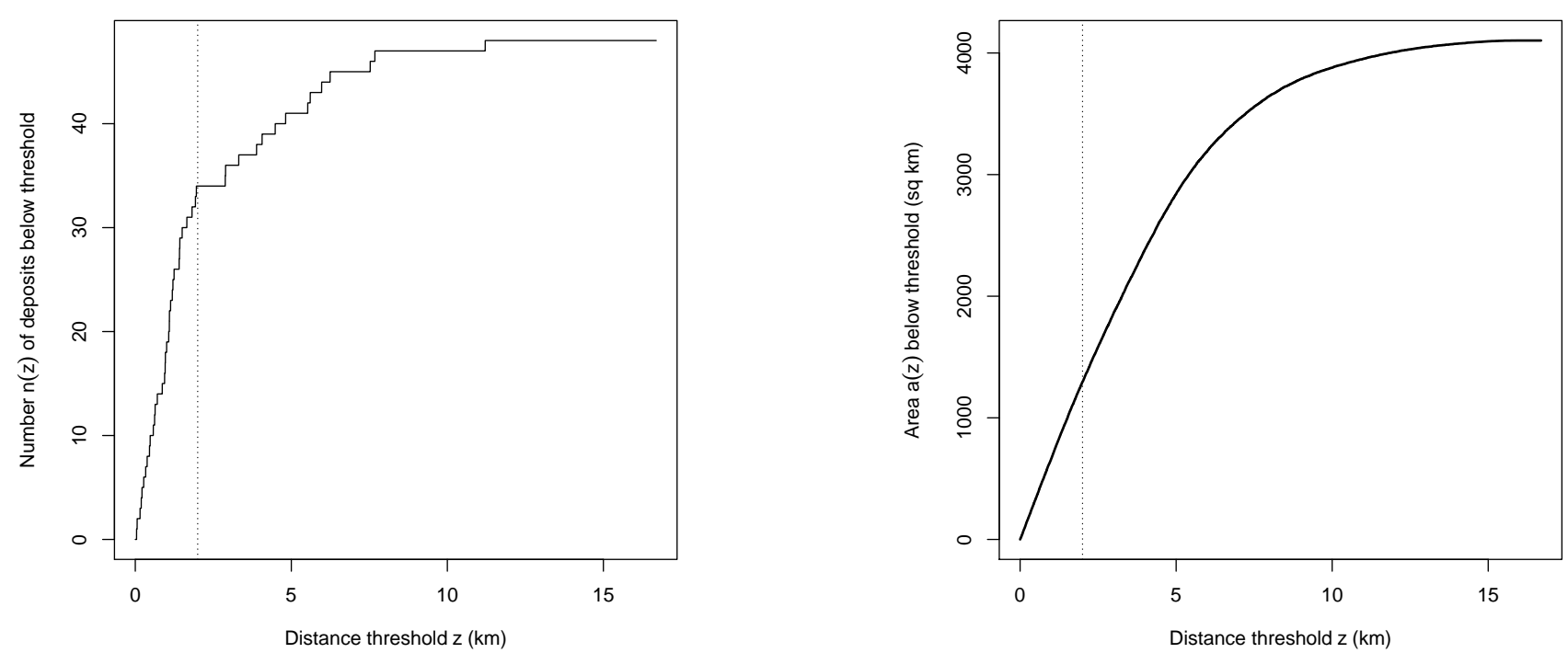

Fig. 6 Essential data for selecting the best threshold in the synthetic data example of Figure 5. Left: the number $n(z)$ of deposits lying no further than distance $z$ away from the nearest lineament, plotted against $z$. Right: the area $a(z)$ of the buffer consisting of all locations lying no further than distance $z$ away from the nearest lineament, plotted against $z$. Dotted vertical lines show the correct value of distance threshold, $z_{\text {true }}=2 \mathrm{~km}$.

\begin{tabular}{|l|l|}
\hline SYMBOL & MEANING \\
\hline$S$ & survey region \\
$a$ & area of survey region \\
$n$ & total number of deposits \\
$x_{1}, \ldots, x_{n}$ & spatial locations of deposits $1,2, \ldots, n$ \\
$Z$ & predictor variable (a function of spatial location) \\
$u$ & any spatial location \\
$Z(u)$ & value of predictor $Z$ at location $u$ \\
$z$ & any candidate value of threshold for the predictor \\
$n(z)$ & number of grid cells containing deposits where $Z \leq z$ \\
$a(z)$ & area of region of survey where $Z \leq z$ \\
$s(z)$ & area fraction $s(z)=a(z) / a$ \\
$\hat{z}$ & selected (estimated) threshold value \\
\hline
\end{tabular}

Table 1 Summary of mathematical notation.

is not standard; we have chosen a more mnemonic notation. The performance of these criteria is summarised in Table 10 in the Discussion section.

\begin{tabular}{|l|l|c|}
\hline SYMBOL & MEANING & EQUATION \\
\hline$\widehat{C}(z)$ & WofE contrast & $(2)$ \\
$t(z)$ & Studentised contrast & $(4)$ \\
$X^{2}(z)$ & $\chi^{2}$ test statistic & $(6)$ \\
$Y(z)$ & Youden criterion & $(8)$ \\
$\ln L(z)$ & profile log-likelihood & $(12)$ \\
$\operatorname{AR}(z)$ & Akman-Raftery criterion & $(13)$ \\
\hline
\end{tabular}

Table 2 Notation for threshold selection criteria, and equation numbers for their definitions. Each criterion is expressed as a function of the candidate threshold value $z$.

\section{THRESHOLD SELECTION USING WOFE CONTRAST}

Threshold selection using the WofE contrast (with or without rescaling) is expounded by Bonham-Carter (1995, Section 9.5, pp. 319-325) and is widely used.

In the original method, the threshold is selected by "progressively altering the threshold value to seek the greatest contrast" (Goodacre et al. 1993, pp. 291-292), that is, by choosing the threshold value which maximises 
the WofE contrast (Bonham-Carter 1995, pp. 321-322; Agterberg 2014, p. 150). Recent examples include Cheng (2008, Section 7.2.3), Romero-Calcerrada and Luque (2006) and Romero-Calcerrada et al. (2010).

As a function of the candidate threshold $z$, the estimated WofE contrast (1) is given by

$$
\widehat{C}(z)=\ln \left(\frac{n(z)}{a(z)} / \frac{n-n(z)}{a-a(z)}\right)=\ln \frac{n(z)}{a(z)}-\ln \frac{n-n(z)}{a-a(z)},
$$

that is, in (1) we replace $n_{B}$ by $n(z)$ and $a_{B}$ by $a(z)$, so that $n_{\bar{B}}=n-n_{B}=n-n(z)$ and $a_{\bar{B}}=a-a_{B}=a-a(z)$, giving (2). This formula assumes grid cell area is negligible; the corresponding formula for non-negligible cell area is given in the appendix entitled Non-negligible grid cell size as equation (42).

The left panel of Figure 7 shows a plot of the estimated contrast $\widehat{C}(z)$ against the candidate threshold distance $z$ for the synthetic data of Figure 5. The estimated threshold is $\widehat{z}=7.68$, which is a poor estimate of the correct threshold $z_{\text {true }}=2 \mathrm{~km}$. However, very close to the correct threshold value, the graph has a secondary peak, and this would have been selected if the range of distances $z$ had been suitably restricted. In particular, to avoid instability associated with small counts, it may be appropriate to restrict $z$ to the range where both the expected counts $n a(z) / a$ and $n(1-a(z) / a)$ are greater than or equal to 5 , as discussed below equation (20).
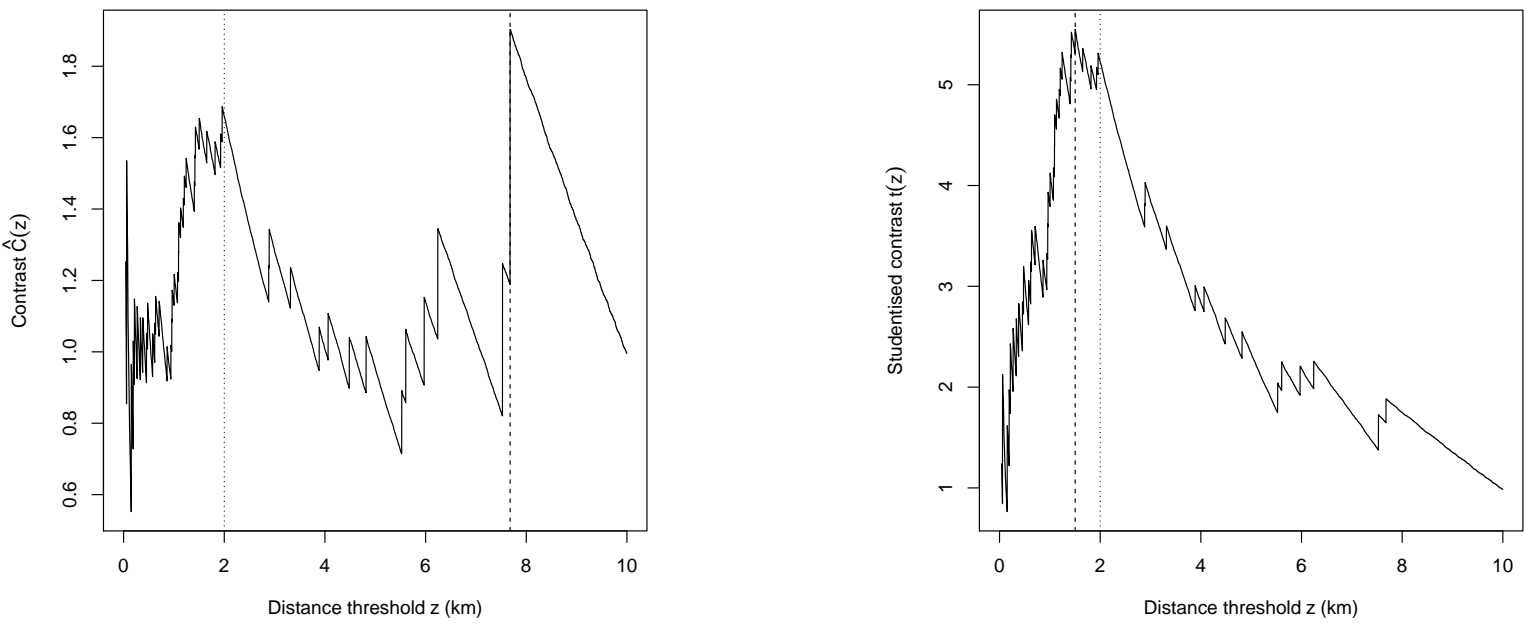

Fig. 7 Weights-of-evidence contrast (Left) and Studentised contrast (Right) plotted against candidate threshold distance, for the synthetic data of Figure 5 . Dotted vertical lines show the correct threshold, $z_{\text {true }}=2 \mathrm{~km}$. Dashed vertical lines show estimated thresholds, $\widehat{z}=7.68 \mathrm{~km}$ and $\widehat{z}=1.50 \mathrm{~km}$, respectively.

For efficiency this procedure can be streamlined. Although our algorithm has considered a finely spaced grid of candidate values $z$, this is not strictly necessary. The estimated contrast $C(z)$ will be maximised at one of the values $z_{1}, \ldots, z_{n}$ which are the predictor values at the deposit locations (for example, the distances from each deposit location to the nearest fault), because the cumulative count $n(z)$ is incremented only at these values (assuming $a(z)$ is a strictly increasing function of $z$ ). Accordingly, a more efficient algorithm would involve computing only $C\left(z_{1}\right), \ldots, C\left(z_{n}\right)$ and determining which of these is greatest.

We strongly recommend the use of graphical plots like Figure 7 as a tool for evaluating the evidence, identifying quirks of the data, and detecting nonsensical or geologically implausible results. If prior geological knowledge is available, then these graphs and numerical procedures can be restricted to the range of threshold values $z$ which are geologically plausible.

\section{THRESHOLD SELECTION USING STUDENTISED CONTRAST}

The statistical performance of the contrast, as an index for selecting the threshold, becomes erratic at the extreme ends of the range of thresholds, because of sampling variability. It is natural to adjust for the sampling variability, as explained below. 


\section{Binary Predictor}

Returning for a moment to the context of a binary predictor, the estimated standard error of the WofE contrast $\widehat{C}$ is

$$
\operatorname{se}(\widehat{C})=\sqrt{\frac{1}{n_{B}}+\frac{1}{n_{\bar{B}}}} .
$$

This is the asymptotic (large sample) formula for standard error, derived using the "delta method" or "propagation of error" (Lehmann 1999, Section 2.5, pp. 85-93; Wasserman 2004, Section 9.9, pp. 131-132; Rice 2006, Section 4.6, pp. 149-154) assuming independence of outcomes in each grid cell, and assuming cell areas are negligible. It is reasonably accurate when both the numbers $n_{B}$ and $n_{\bar{B}}$ are greater than or equal to 5 (implying that the total number $n$ must be at least 10). The formula (3) is valid for any feature $B$. See the appendix headed Non-negligible Grid Cell Size when grid cell areas are large.

The "Studentised contrast" is the ratio of the contrast to its standard error, $t=\widehat{C} / \operatorname{se}(\widehat{C})$ (Bonham-Carter 1995, p. 325; Cheng 2008, Section 7.2.3; J. Liu and Cheng 2019; Cheng 2007; Porwal et al. 2010, Table 1, p. 187). A value of $t$ greater than 2 can be considered large. Although the term "Studentised" is commonly used when a variable is divided by its standard error, it may be misleading here, because the rescaled variable $t$ does not follow a Student's $t$ distribution in this context. This is discussed further in the section SIGNIFICANCE TESTS.

For the synthetic data in the right panel of Figure 5 , the deposit counts are $n_{B}=34$ and $n_{\bar{B}}=14$, so the estimated standard error according to $(3)$ is $\operatorname{se}(\widehat{C})=\sqrt{1 / 34+1 / 14}=0.32$. The "Studentised contrast" is $t=\widehat{C} / \operatorname{se}(\widehat{C})=1.66 / 0.32=5.19$.

\section{Numerical Predictor}

For a numerical predictor $Z$ as discussed in the section on THE THRESHOLDING PROBLEM, we consider the Studentised contrast as a function of the candidate threshold value $z$,

$$
t(z)=\frac{\widehat{C}(z)}{\operatorname{se}(\widehat{C}(z))},
$$

where the standard error (3) of the estimated contrast is

$$
\operatorname{se}(\widehat{C}(z))=\sqrt{\frac{1}{n(z)}+\frac{1}{n-n(z)}} .
$$

Again this assumes grid cell areas are negligible; see the appendix Non-negligible Grid Cell Size for the more general formula (44).

The right panel of Figure 7 is a graph of the Studentised contrast $t(z)$ for the synthetic data against the candidate threshold distance $z$. The estimated threshold obtained by maximising the Studentised contrast is $\widehat{z}=1.50 \mathrm{~km}$, which is a mediocre estimate of the correct threshold $z_{\text {true }}=2 \mathrm{~km}$, but is an improvement on the threshold chosen using the unadjusted contrast.

\section{THRESHOLD SELECTION USING THE $\chi^{2}$ STATISTIC}

Another way to evaluate the strength of evidence for a threshold is to use a statistical significance test. The $\chi^{2}$ test is described in the section SIGNIFICANCE TESTS. Bonham-Carter and Agterberg (1990) used the test statistic associated with the $\chi^{2}$ test as an alternative criterion for threshold selection. Statistical theory would suggest that this is desirable, because the $\chi^{2}$ test is valid for smaller samples (fewer deposits) than a test based on the Studentised contrast. This method has been demonstrated on data from the Yilgarn craton by Brown (2002) and Brown et al. (2002).

As a function of the candidate threshold value $z$, the $\chi^{2}$ test statistic (equations (18) and (19)) is

$$
X^{2}(z)=n \frac{\left(\frac{n(z)}{n}-\frac{a(z)}{a}\right)^{2}}{\frac{a(z)}{a}\left(1-\frac{a(z)}{a}\right)},
$$


while the signed square root $(20)$ is

$$
X(z)=\sqrt{n} \frac{\left(\frac{n(z)}{n}-\frac{a(z)}{a}\right)}{\sqrt{\frac{a(z)}{a}\left(1-\frac{a(z)}{a}\right)}} .
$$

Note that these formulae do not assume that the grid cell area is negligible.

Figure 8 shows the $\chi^{2}$ test statistic $X^{2}(z)$ plotted against candidate threshold distance for the synthetic data example. The estimated threshold obtained by maximising the $\chi^{2}$ test statistic is $\widehat{z}=1.50 \mathrm{~km}$, agreeing with the result from the statistic $t$. Again, this is a mediocre estimate.

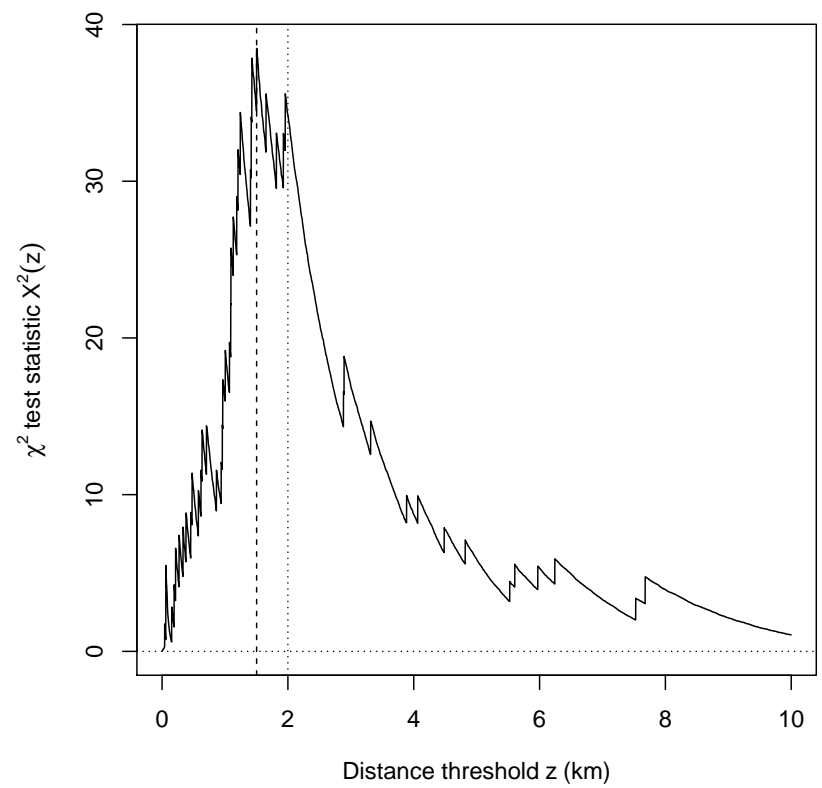

Fig. $8 \chi^{2}$ test statistic plotted against candidate threshold distance, for the synthetic data of Figure 5 . Dotted vertical lines show the correct threshold, $z_{\text {true }}=2 \mathrm{~km}$. Dashed vertical lines show estimated threshold $\widehat{z}=1.50 \mathrm{~km}$.

Values of $X^{2}(z)$ at each end of the graph are highly unreliable because the $\chi^{2}$ test is valid only when the expected count $n a(z) / a$ lies between 5 and $n-5$, as discussed below equation (20).

\section{THRESHOLD SELECTION USING THE CAPTURE-EFFICIENCY CURVE}

This section reviews the role of the capture-efficiency curve, offering new insights and highlighting its central and unifying role in threshold selection.

\section{Principle}

The methods discussed in this section are based on comparing the distributions of predictor values at the deposit and non-deposit locations.

Two standard statistical ways to perform this comparison are illustrated in Figure 9 for the synthetic data example. In the left panel, the two curves show the cumulative fraction $F(z)=n(z) / n$ of deposits where the predictor value is less than or equal to a threshold value $z$, and the fraction of area $G(z)=a(z) / a$ of all spatial locations where the predictor is less than or equal to the same threshold $z$, plotted against the candidate threshold value $z$. If deposits were randomly and homogeneously scattered, these two curves would agree, apart from sampling variation in the counts of deposits. These curves are the cumulative distribution functions of the predictor values for two populations, the deposit and non-deposit locations. That is, $F(z)$ is the cumulative distribution function of the predictor value at a randomly-selected deposit, while $G(z)$ is the cumulative distribution function of the 
predictor value at a randomly-selected location in the study region. In the synthetic data example, the predictor is the distance to the nearest fault, so that $F(z)$ is the cumulative distribution function of the distance from a randomly-selected deposit to the nearest fault, while $G(z)$ is the cumulative distribution function of the distance from a randomly-selected spatial location to the nearest fault.
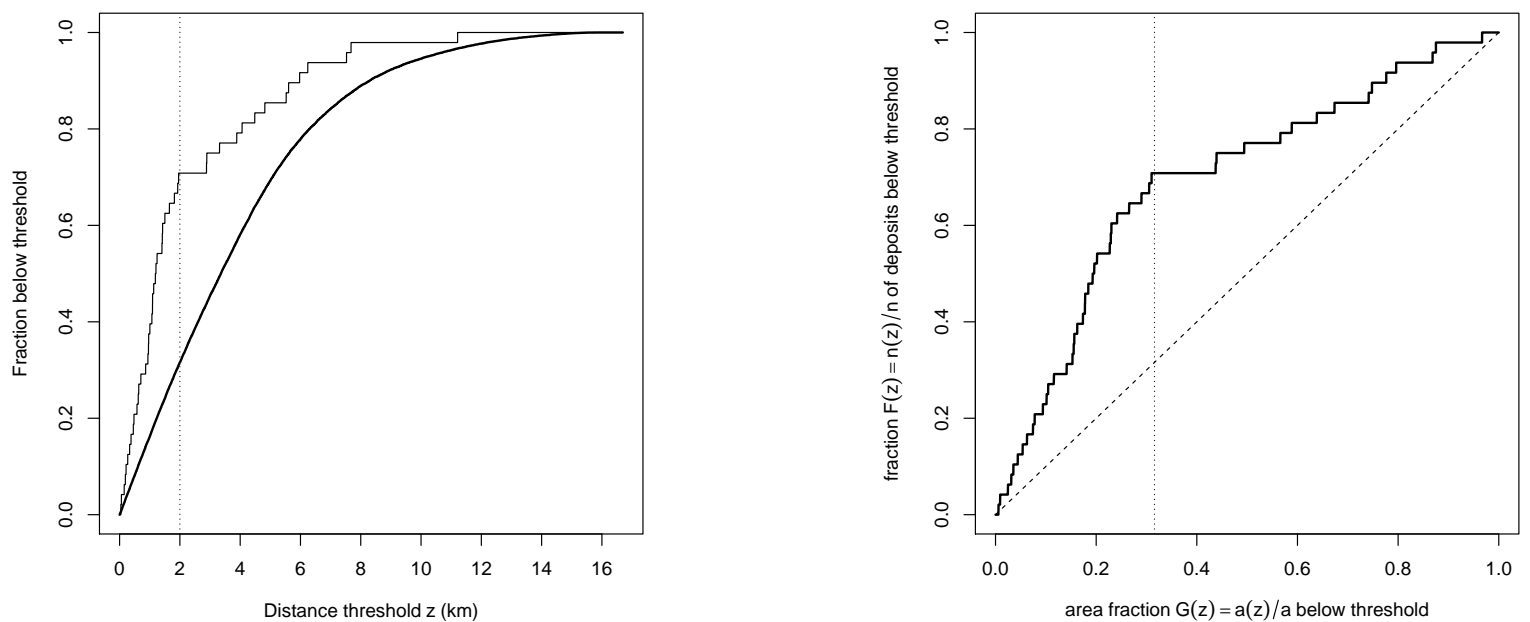

Fig. 9 Distributional comparisons, illustrated using the synthetic data example. Left: the fraction $F(z)=n(z) / n$ of deposits at or below threshold (thin solid line) and the fraction of area $G(z)=a(z) / a$ at or below threshold (thick solid line), plotted against candidate threshold value $z$. Dotted vertical line shows correct threshold $z_{\text {true }}=2 \mathrm{~km}$. Right: capture-efficiency curve. Thick solid line is the fraction $F(z)=n(z) / n$ of deposits at or below threshold plotted against fraction of area $G(z)=a(z) / a$. Dotted vertical line shows area fraction $G\left(z_{\text {true }}\right)=1296 / 4102=0.32$ corresponding to the correct threshold $z_{\text {true }}$. Diagonal dashed line would correspond to complete agreement between $F(z)$ and $G(z)$, which would occur if deposits were uniformly scattered over the study region.

In the right panel of Figure 9, the single curve shows the fraction of deposits $F(z)=n(z) / n$ plotted against the corresponding fraction of area $G(z)=a(z) / a$ for various candidate values $z$ of the threshold. If deposits were randomly and homogeneously scattered, this curve would agree with the diagonal straight line $y=x$ apart from sampling variation. Such a curve has been reinvented many times and is variously known as a probability-probability (P-P) plot (Wilk and Gnanadesikan 1968), ROC curve (Krzanowski and Hand 2009; Nam and D'Agostino 2002), capture-efficiency curve (Porwal et al. 2010) or fitting-rate curve (Fabbri and Chung 2008). We shall use the term "capture-efficiency curve", which is more widely recognised in mineral prospectivity analysis. ${ }^{3}$ The captureefficiency curve and the ROC curve are indistinguishable if grid cell area is negligible, but they are slightly different otherwise.

The capture-efficiency curve depicts the proportion of deposits captured by cumulative proportions of the study area. There are several important reasons for using this curve to select the threshold. The first reason is that the capture-efficiency curve removes spatial geometry effects. In the left panel of Figure 9, the shapes of the two curves depend on the spatial arrangement of the fault lines in Figure 5; the area $a(z)$ of the buffer is not a simple function of the buffer distance $z$, because it depends on the spatial arrangement of the faults. In the right panel of Figure 9 the effect of geometry is removed; a uniform distribution of deposits would correspond to the diagonal straight line in the right panel. Moreover, the capture-efficiency curve is unchanged even if the predictor is subjected to a transformation - for example, if distance-to-nearest-fault is replaced by the squared distance, square root of distance, or any increasing function of distance.

The second reason for using the capture-efficiency curve is that, if a simple threshold exists, the curve will have an inverted "V" shape, and the position of the elbow corresponds to the threshold. This is discussed in the next sub-section. The point of significant inflexion, where the slope changes from steep to gentle, can be used as a threshold for separating high-prospectivity areas from low-prospectivity areas (Porwal et al. 2010).

The capture-efficiency curve can also be interpreted as a cumulative distribution function in its own right, which has useful implications, explained in the appendix Capture-Efficiency Curve as a CDF.

${ }^{3}$ D. Harris and Pan (1999) used a related measure of classification performance which they call exploration performance. Exploration is viewed as the task of selecting the optimum threshold for allocating grid cells to the mineralized class, and it is assumed that all grid cells classified by the system as mineralized are retained as ground for further exploration. Classification performance is measured by the percentage of total grid cells that must be retained to ensure that different percentages of mineralized grid cells are retained when various cutoff probabilities for mineralization are applied to neural network output. 


\section{Relation to Threshold}

The capture-efficiency curve gives the clearest indication of existence of a threshold (Goodacre et al. 1993; Porwal et al. 2010) for the following reason.

Suppose that the predictor variable $Z$ does indeed have a threshold-like effect. That is, there is a true threshold value $z_{\text {true }}$ so that, in the region where $Z \leq z_{\text {true }}$, deposits are homogeneous with expected density $\mu_{1}$ deposits per unit area, while in the complementary region where $Z>z_{\text {true }}$, deposits are homogeneous with expected density $\mu_{0}$ per unit area. If this true or correct threshold $z_{\text {true }}$ were chosen, the data would conform to a WofE model with true contrast $C_{\text {true }}=\ln \left(\mu_{1} / \mu_{0}\right)$.

If this simple threshold model is true, then the capture-efficiency curve is expected to be linear on each side of the true threshold. That is, ignoring sampling variability, the capture-efficiency curve should consist of a straight line between the origin $(0,0)$ and the point $P$, and another straight line between $P$ and the top corner $(1,1)$, where $P$ has coordinates $\left(a\left(z_{\text {true }}\right) / a, n\left(z_{\text {true }}\right) / n\right)$.
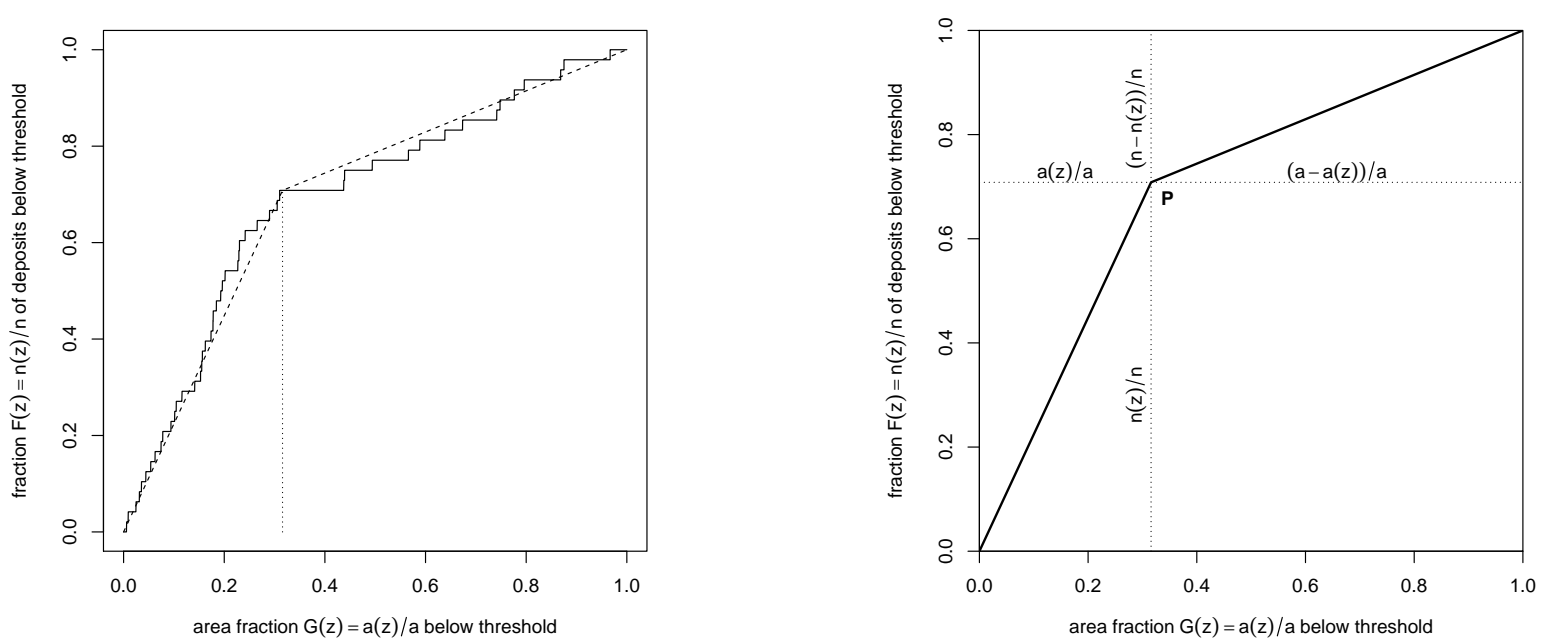

Fig. 10 Use of the capture-efficiency curve. Left: capture-efficiency curve (solid line) for synthetic data of Figure 5 and straight line approximation (dashed line) on either side of the point of maximum change in slope (dotted vertical line). Right: diagram for calculation (see text).

The synthetic data example conforms to this simple model, and its capture-efficiency curve (in the right panel of Figure 9) is approximately linear on each side of the true threshold value $z_{\text {true }}=2 \mathrm{~km}$. The left panel of Figure 10 shows the same capture-efficiency curve augmented by the two lines described above, using knowledge of the true threshold $z_{\text {true }}=2 \mathrm{~km}$.

There is also a connection with estimation of the WofE contrast: the slopes of the initial and final segments of the capture-efficiency curve (or in general, the ROC curve) are the average densities of deposits on either side of the threshold. The logarithm of the ratio of these slopes is equal to $\widehat{C}\left(z_{m}\right)$, the estimate of the WofE contrast at the elbow point $z_{m}$. We shall explain this in detail, referring to the right panel of Figure 10. For any point $(x, y)$ on the capture-efficiency curve, consider the two straight lines joining $(0,0)$ to $(x, y)$ and joining $(x, y)$ to $(1,1)$ as illustrated in Figure 10. These lines have slope $y / x$ and $(1-y) /(1-x)$ respectively. The logarithm of the ratio of the two slopes is $\ln (y / x)-\ln ((1-y) /(1-x))$. Since the point $(x, y)$ lies on the capture-efficiency curve, its coordinates are $x=a(z) / a$ and $y=n(z) / n$ for the corresponding candidate threshold $z$, so the logarithm of the ratio of the two slopes is equal to (2), the estimated contrast $\widehat{C}(z)$.

It follows that a technique which selected the threshold $z$ by maximising the ratio of the slopes of the two lines, as described above, would be equivalent to maximising the WofE contrast.

\section{Youden Criterion}

If deposits were uniformly scattered over the study region, then the fraction $F(z)=n(z) / n$ of deposits below a certain threshold $z$ would be approximately equal to the fraction $G(z)=a(z) / a$ of study area below this threshold. 
Discrepancies between the "observed" fraction $F(z)$ and the "expected" fraction $G(z)$ constitute evidence against uniform scattering.

If the simple threshold model is true, as described in the previous section, then the estimated threshold $\hat{z}$ can be obtained as the position of greatest discrepancy between the observed fraction $F(z)$ and "expected" fraction $G(z)$. This location can be identified in several ways. In the left panel of Figure 9 , the threshold $\hat{z}$ is the location where the two curves have the greatest vertical separation, that is, the location $z$ where $F(z)-G(z)$ is greatest. In the right panel of Figure 9, the threshold $\hat{z}$ corresponds to the location where the capture-efficiency curve has the greatest vertical deviation from the diagonal line; the $x$ coordinate of this location is the area fraction $G(\widehat{z})=a(\widehat{z}) / a$ and from this we can determine the estimated threshold value $\widehat{z}$. A third, graphical technique for identifying the estimated threshold is described below (see Figure 12).

The vertical deviation at a candidate threshold value $z$ is

$$
Y(z)=F(z)-G(z)=\frac{n(z)}{n}-\frac{a(z)}{a} .
$$

The maximum positive vertical deviation is

$$
Y=\max _{z} Y(z)
$$

Under the assumption that grid cell areas are negligible, $Y$ is equivalent to the Youden index (Chen and Wu 2019; Ruopp et al. 2008; Youden 1950) of classifier performance, and we call (8) the Youden criterion. ${ }^{4}$ The general formula for $Y(z)$, not assuming that grid cell area is negligible, is given in the appendix Non-negligible Grid Cell Size as equation (45).

The Youden index was originally defined (Youden 1950) as a measure of optimal performance of a medical diagnostic classifier. As explained by Ruopp et al. (2008), suppose that a patient's disease status were reported as positive (disease present) if the result of a biomedical assay returned a value lower than a specified cutoff, and otherwise were reported as negative (disease absent). Sensitivity (Se) and specificity (Sp) are the probabilities of correctly identifying diseased and non-diseased individuals, respectively. If equal importance is given to sensitivity and specificity, then the predictive power or discriminating ability of the technique can be measured by Se-(1-Sp) and the cutoff should be chosen to maximise this quantity. The maximised value is the Youden (1950) index.

In prospectivity analysis, the analogous procedure (Chen and Wu 2019) is to label a grid cell as "prospective" when the value of the predictor variable $Z$ is below a threshold $z$. The sensitivity Se is the probability of correctly identifying grid cells that contain deposits, that is, the probability that a grid cell which actually contains a deposit will be correctly labelled as prospective by this rule. Under our assumption that grid cell areas are negligible, the sensitivity is equal to $n(z) / n=F(z)$, the fraction of deposit locations where the predictor is less than or equal to $z$. The specificity $\mathrm{Sp}$ is the probability of correctly identifying grid cells that do not contain a deposit, and is equal to the fraction $1-G(z)=[a-a(z)] / a$ of study area where the predictor value exceeds $z$. The discriminating power of the classifier, Se $-(1-\mathrm{Sp})$, is equal to $F(z)-G(z)$.

Figure 11 shows the calculation of the Youden criterion for the synthetic data example. The left panel shows the two cumulative distribution functions $F(z)=n(z) / n$ and $G(z)=a(z) / a$ plotted against candidate threshold distance $z$. The right panel shows the Youden criterion $Y(z)=F(z)-G(z)$ plotted against $z$. In this synthetic data example, the estimated threshold obtained by maximising the Youden criterion is $z=1.96 \mathrm{~km}$, an excellent estimate of the true value $z_{\text {true }}=2 \mathrm{~km}$.

In statistical terminology, the graph of the Youden criterion in the right panel of Figure 11 is a "control chart" (Shewhart 1983) of the CUSUM type (Page 1954) and these are known to perform well for estimation of change-point locations (Basseville and Nikiforov 1993, Chapter 2; Barnard 1959; Hinkley 1971).

An alternative graphical technique for identifying the estimated threshold by the Youden method is sketched in Figure 12. Parallel lines with slope equal to 1 are drawn above the capture-efficiency curve, and displaced downward until a line touches the curve. The tangent point determines the selected threshold: from the tangent point we read off the horizontal coordinate, which is equal to the optimal area fraction, and this corresponds to the optimal threshold for the predictor.

In the original definition of the Youden index as a measure of diagnostic performance, equal importance was given to sensitivity and specificity. In the context of mineral prospectivity, this corresponds to assigning a monetary figure to the cost of thoroughly exploring each $1 \%$ of survey area that is equal to the value of each $1 \%$ of existing mineral deposits. If a different cost-benefit ratio $\alpha$ had been used, so that the value of $1 \%$ of mineral deposits was equal to the cost of testing $\alpha \%$ of the survey area, this would correspond to using parallel lines of slope $\alpha$ instead of slope 1 in Figure 12. A valuable insight from Figure 12 is that we would have selected the same threshold even if we had used parallel lines with some other slopes, because of the inverted "V" shape of the capture-efficiency

${ }^{4}$ The Youden index is traditionally denoted by the letter $J$, but we have called it $Y$ for a more mnemonic notation. 

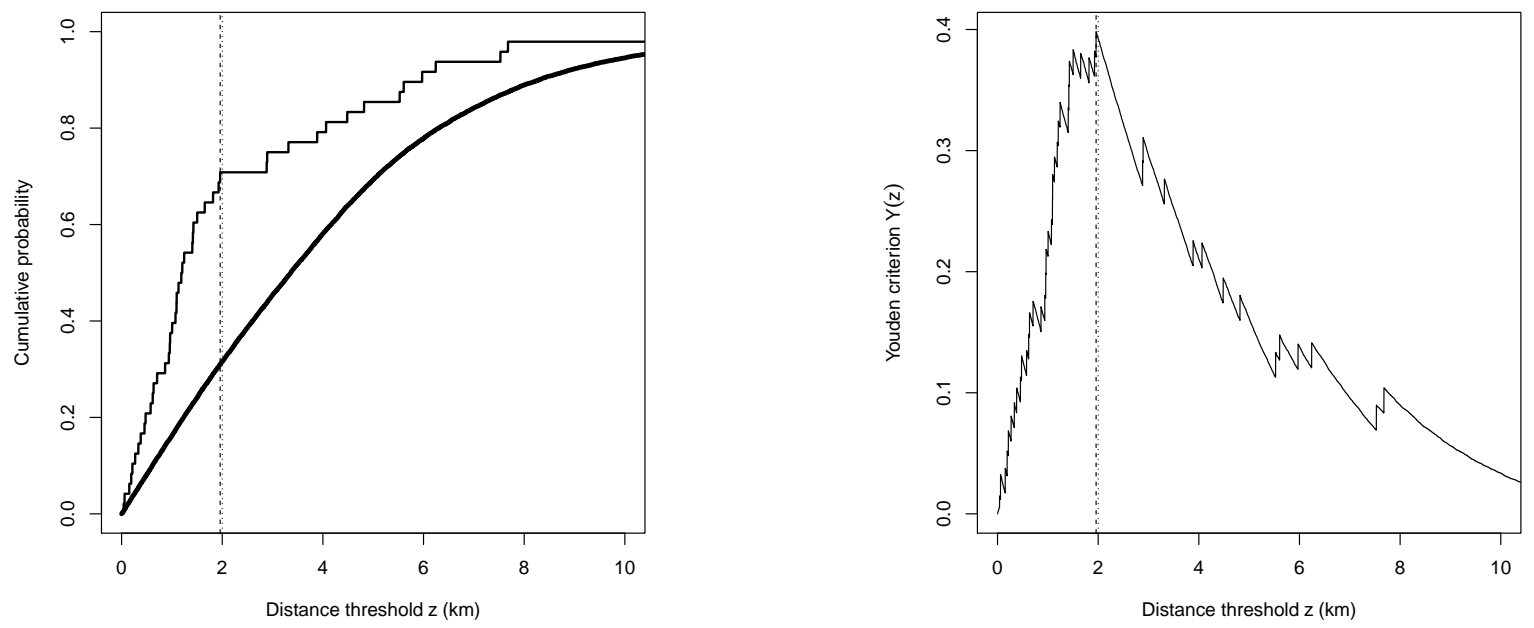

Fig. 11 Youden criterion for the synthetic data of Figure 5. Left: cumulative fractions $n(z) / n$ (thin curve) and $a(z) / a$ (bold curve) plotted against candidate threshold distance $z$. Right: Youden criterion $Y(z)=n(z) / n-a(z) / a$ plotted against $z$. Vertical line shows the correct threshold, $z_{\text {true }}=2 \mathrm{~km}$ and the estimated threshold, $\widehat{z}=1.96 \mathrm{~km}$, which are visually indistinguishable.

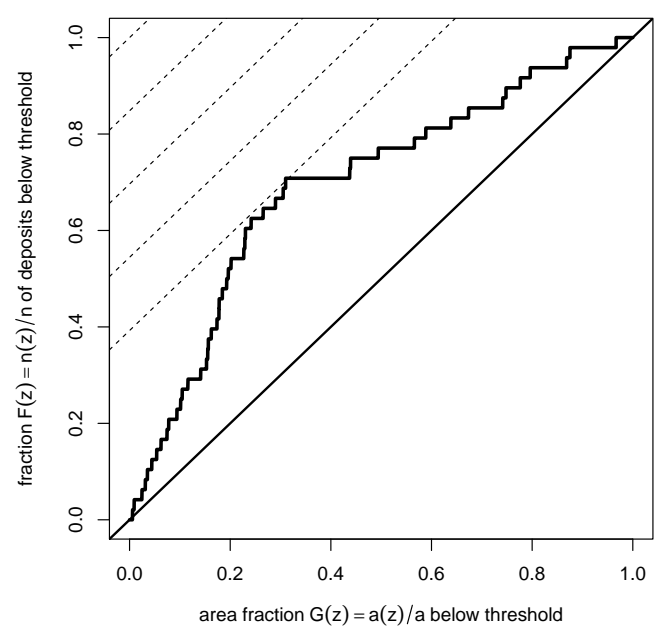

Fig. 12 Alternative graphical technique for the Youden method. The capture efficiency curve for the synthetic data is drawn. A line with slope 1 (dashed) is moved down the graph until it intersects the capture efficiency curve. The tangent point corresponds to the threshold that maximises the Youden criterion.

curve. That is, when the simple threshold model is true, the cost-benefit ratio is not very influential in choosing the threshold.

\section{Slope of Curve}

We have seen that, if the simple threshold model is true, the capture-efficiency curve is expected to be linear on each side of the threshold, and the threshold position can be discerned by any of the procedures described above. It would be more realistic to assume that the threshold model is not exactly true, but that there is nevertheless a large difference between the densities of deposit points occurring at low and high values of the predictor variable. In that case, one can exploit the fact that, at any position along the capture-efficiency curve, say the point $(a(z) / a, n(z) / n)$, the slope of the curve is proportional to the probability of a deposit when the predictor value is equal to $z$ (Baddeley et al. 2012; Goodacre et al. 1993; Porwal et al. 2010). It would then be appropriate to select the threshold by finding "the point of significant inflexion where the slope changes from steep to gentle" (Porwal et al. 2010, p. 193). However, the procedure to be used for finding the inflection point or for measuring changes of slope was not described in detail in the paper by Porwal et al. (2010). 
A possible weakness of this method is that estimates of derivatives can be highly unstable, and some form of smoothing is required. Goodacre et al. (1993) used spline smoothing to estimate the local slope of the captureefficiency curve. The closely related technique of Baddeley et al. (2012) used kernel smoothing.

\section{THRESHOLD SELECTION USING CHANGE-POINT ANALYSIS}

In statistical theory, an abrupt change in the characteristics of a signal is called a change-point (Basseville and Nikiforov 1993; Page 1957; Zacks 1983). Detection of abrupt change, and estimation of the location of the changepoint, are challenging problems because they do not conform to the standard assumptions of differentiability that justify most statistical techniques.

From the viewpoint of this literature, it would be expected that a procedure which estimates the changepoint location by maximising the estimated contrast $\widehat{C}$ would not perform well. The estimated contrast $\widehat{C}(z)$ is an estimate of effect size, that is, an estimate of the magnitude of the change at $z$, rather than a measure of the evidence for a change at location $z$. Adjusting for the variability of $\widehat{C}(z)$ could improve performance, so the Studentised contrast $t(z)$ would be expected to perform better. As remarked above, the Youden criterion $Y(z)$ would be expected to perform well, because it can be regarded as a control chart of CUSUM type, and these are known to perform well for estimation of change-point locations (Basseville and Nikiforov 1993, Chapter 2; Barnard 1959; Hinkley 1971). For testing statistical significance of a change-point, it is important that the Youden criterion is associated with the Kolmogorov-Smirnov test (see the subsection on the Kolmogorov-Smirnov test in the section SIGNIFICANCE TESTS below).

\section{Profile Likelihood}

A common way to estimate the threshold $z_{\text {true }}$ would be to maximise the profile likelihood.

\section{Likelihood}

Likelihood (Fisher 1922) is a fundamental tool in statistical analysis. For a parametric statistical model, the likelihood is a function $L(\theta)$ of the model parameters $\theta$; it is defined as the probability of observing the data that were actually observed, assuming the model is true with the specified parameter values. The likelihood function serves as a comparative measure of the support given by the data to different choices of the parameter values.

One important use of the likelihood function is to estimate the model parameters from the data. In the method of maximum likelihood, after observing the data we select the parameter value which maximises the likelihood, that is, the parameter value which enjoys the greatest support given by the data.

Expositions of likelihood can be found in Hogg and Craig (1970, Section 8.2), Rice (2006, Section 8.5), Lindsey (1996, Chapter 3), Severini (2000, Chapter 3), Kalbfleisch (1985, Chapters 9-10) and Murphy and van der Vaart (2000).

In prospectivity analysis, the techniques of logistic regression and WofE are both applications of the method of maximum likelihood (Baddeley et al. 2010; Schaeben 2014). Furthermore, the hypothesis tests reviewed in the section on SIGNIFICANCE TESTS are all obtained from the likelihood.

Maximising the likelihood is equivalent to maximising the logarithm of the likelihood, and it is usual to work with the log-likelihood for convenience. For further simplification, any terms in the log-likelihood equation that do not depend on the parameters can be removed because they do not affect the maximisation.

For the simple threshold model, the observed data are the two curves depicted in Figure 6, and the loglikelihood $^{5}$ of the candidate threshold $z$ and densities $\mu_{0}, \mu_{1}$ is

$$
\ln L\left(z, \mu_{0}, \mu_{1}\right)=n(z) \ln \mu_{1}+(n-n(z)) \ln \mu_{0}-a(z) \mu_{1}-(a-a(z)) \mu_{0} .
$$

The maximum likelihood estimate of the three parameters $\left(z, \mu_{0}, \mu_{1}\right)$ would be obtained by finding the values of $\left(z, \mu_{0}, \mu_{1}\right)$ which yield the maximum value of $(10)$.

\footnotetext{
${ }^{5}$ For a detailed derivation of this likelihood, see the appendix Likelihood Function for Threshold Model. For a different, more intuitively accessible explanation, see Baddeley et al. (2015, pp. 132-135, 342-343).
} 


\section{Profile Likelihood for the Threshold Model}

The profile likelihood method is a general technique for dealing with multiple parameters when one parameter is more "important" than the others. The profile likelihood is a function of the "important" parameter only, and is defined as the maximum value of likelihood over all possible values of the "less important" parameters.

For the threshold model, the "important" parameter is the threshold value $z$. To apply profile likelihood to the threshold model, for each candidate threshold value $z$ we maximise the likelihood over all possible values of the densities $\mu_{0}, \mu_{1}$. Using calculus the maximum is achieved when $\mu_{1}=n(z) / a(z)$ and $\mu_{0}=(n-n(z)) /(a-a(z))$; these are just the average densities of deposits per unit area inside and outside the buffer, as we might expect. Substituting these into (10) we obtain the profile log-likelihood for a candidate threshold $z$,

$$
\ln L(z)=n(z) \ln \frac{n(z)}{a(z)}+(n-n(z)) \ln \frac{n-n(z)}{a-a(z)}-n
$$

Using (2) this is equivalent to

$$
\ln L(z)=n \ln \frac{n(z)}{a(z)}-(n-n(z)) \widehat{C}(z)-n
$$

Maximising the profile log-likelihood over candidate threshold values $z$ is equivalent to maximising the likelihood of the three parameters $\left(z, \mu_{0}, \mu_{1}\right)$, enabling us to estimate the threshold by the method of maximum likelihood.

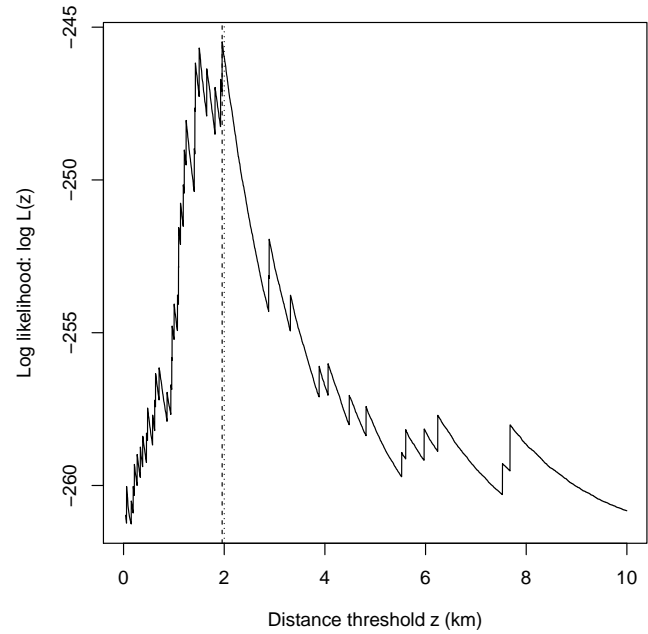

Fig. 13 Log-likelihood plotted against candidate threshold distance, for the synthetic data of Figure 5 . Dotted vertical line shows correct threshold, $z_{\text {true }}=2 \mathrm{~km}$. Dashed vertical line shows estimated threshold, $\widehat{z}=1.96 \mathrm{~km}$, virtually indistinguishable from the correct value.

Figure 13 shows the application of likelihood methods to the threshold selection problem for the synthetic data. The profile $\log$-likelihood $\ln L(z)$ from (12) is plotted against candidate threshold distance $z$. The estimated threshold, $\widehat{z}=1.96 \mathrm{~km}$, obtained by maximising the profile log-likelihood, delivers an excellent estimate of the correct value, $z=2 \mathrm{~km}$. In particular, this method yields a better estimate than is obtained by maximising the Studentised contrast $t$.

Although likelihood methods are theoretically optimal (i.e. they have the best possible statistical performance) in many contexts, they may not be optimal for the threshold model. The standard theory justifying maximum likelihood assumes that the likelihood is a differentiable function of the parameters. This is not true for the threshold model because the log-likelihood (10) or (12) is not differentiable as a function of the candidate threshold $z$. This has the important consequence that maximum likelihood is not necessarily the optimal method for estimating the threshold.

Another important consequence is that standard errors and confidence intervals cannot be calculated in the usual way. In the section BOOTSTRAP STANDARD ERRORS AND CONFIDENCE INTERVALS below, however, we propose a bootstrap method for calculating standard errors and confidence intervals for the threshold location. 


\section{Other Statistical Approaches to Change-Point Estimation}

Since the method of maximum likelihood is not necessarily optimal for estimating a change-point, there is extensive literature aimed at finding better techniques (Basseville and Nikiforov 1993; Bhattacharya and Brockwell 1976; Bhattacharya and Johnson 1968; Chernoff and Zacks 1964; Darkhovsky 1976; Deshayes 1984; Galun and Trifonov 1982; Gardner 1969; Hájek and Rényi 1955; Hinkley 1970, 1971; Kander and Zacks 1966; Pratt 1959; Sen and Srivastava 1975; Smith 1975; Zacks 1983). For surveys, see Basseville and Nikiforov (1993); Pons (2018).

Thresholding is equivalent to estimating a change-point in probability density. Recall that the the captureefficiency curve can be regarded as a cumulative distribution function. The slope of the capture-efficiency curve can therefore be regarded as a probability density function. If the capture-efficiency curve follows the ideal, inverted "V" shape, then its slope jumps abruptly from a high value to a low value, i.e. there is a change-point in the probability density.

Techniques for estimating a change-point in a probability density go back at least as far as Chernoff and Rubin (1956) and Rubin (1961), and there is now extensive literature ${ }^{6}$ on this problem (Kutoyants 1998, Chapter 5, pp. 183-224). Other literature, with possible relevance to prospectivity, includes models of a change-point plus gradual trend (Loader 1992), a change-point in discretised data (West and Ogden 1997), two change-points (Chernoyarov, Kutoyants, and Top 2018), change-points in waiting time (Commenges and Seal 1985), and smoothing-based estimates (Leonard 1978).

\section{Akman-Raftery Method}

Akman and Raftery (1986) and Raftery and Akman (1986) proposed another method for estimating a change-point in the probability density of a variable. For mathematical convenience, they assumed the variable ranges between 0 and 1 , the change-point is a value $s$ lying strictly between 0 and 1 , and that the probability density of the variable is constant on either side of the change-point.

To apply the Akman-Raftery method to the thresholding problem, we must first transform the threshold value $z$ to the change-point coordinate $s$ by setting $s=s(z)=a(z) / a=G(z)$, the fraction of area of the survey where the predictor value does not exceed the threshold $z$. Equivalently, $s$ is the horizontal coordinate in the capture-efficiency curve. Then the Akman-Raftery criterion is defined as ${ }^{7}$

$$
\operatorname{AR}(z)=\sqrt{s(z)(1-s(z))}\left(\frac{n(z)}{s(z)}-\frac{n-n(z)}{1-s(z)}\right)=\sqrt{a(z)(a-a(z))}\left(\frac{n(z)}{a(z)}-\frac{n-n(z)}{a-a(z)}\right) .
$$

The term in parentheses on the right hand side of (13) is the difference between the estimated densities of deposits in the regions above and below the putative threshold $z$. The standard error of this difference, under the null hypothesis that a threshold is absent, is equal to $1 / \sqrt{n s(z)(1-s(z))}$. That is, $\operatorname{AR}(z)$ is $1 / \sqrt{n}$ times the standardised difference between estimated intensities above and below the putative threshold. This is a "standardised" rather than "Studentised" quantity because the standard error is based on the null hypothesis that a threshold is absent.

The change-point is estimated by maximising $|\operatorname{AR}(z)|$. However, when $s=s(z)$ is close to 0 or 1 , the sampling fluctuations in $\operatorname{AR}(z)$ become large. Accordingly Akman and Raftery (1986) stipulate that the maximum should be taken only for area fractions $s$ in the range $\left[s_{0}, s_{1}\right]$ with $0<s_{0}<s_{1}<1$. For example, restricting to the range $[0.1,0.9]$ would mean that the candidate threshold $z$ must lie between the 10th and 90th percentiles of the values of the predictor variable over the study region.

This constraint is impractical in many applications to mineral prospectivity. If the predictor is distance to a map feature like a fault line or lithological boundary, it is not acceptable to exclude the smallest percentiles, as these correspond to the region where the effect of the feature is likely to be greatest. However, we shall study the effect of this constraint.

In our discussion, the Akman-Raftery criterion is the quantity $\operatorname{AR}(z)$ defined in (13) which could be evaluated for any value of $z$, while the Akman-Raftery method (abbreviated ARM) is the procedure which maximises $\operatorname{AR}(z)$ subject to the constraint on $z$.

For the synthetic data, Figure 14 shows the Akman-Raftery criterion $\operatorname{AR}(z)$ plotted against the candidate threshold distance $z$ in the left panel, and plotted against area fraction $s$ in the right panel. Oblique shading covers the predictor values which lie below the 10th percentile or above the 90th percentile of predictor values in the study region. In this example, the maximum value of $\operatorname{AR}(z)$ over all possible thresholds $z$ is achieved at distance

\footnotetext{
${ }^{6}$ For experts in statistics, we note that the central limit theorem does not apply to this problem: the asymptotic law of the log-likelihood is a compound Poisson process, not a Gaussian process (Pflug 1983; Kutoyants 1998, eq. (5.3), p. 184).

${ }^{7}$ Akman and Raftery (1986) denoted their criterion by the letter $Y$, but we use AR for a more mnemonic notation.
} 

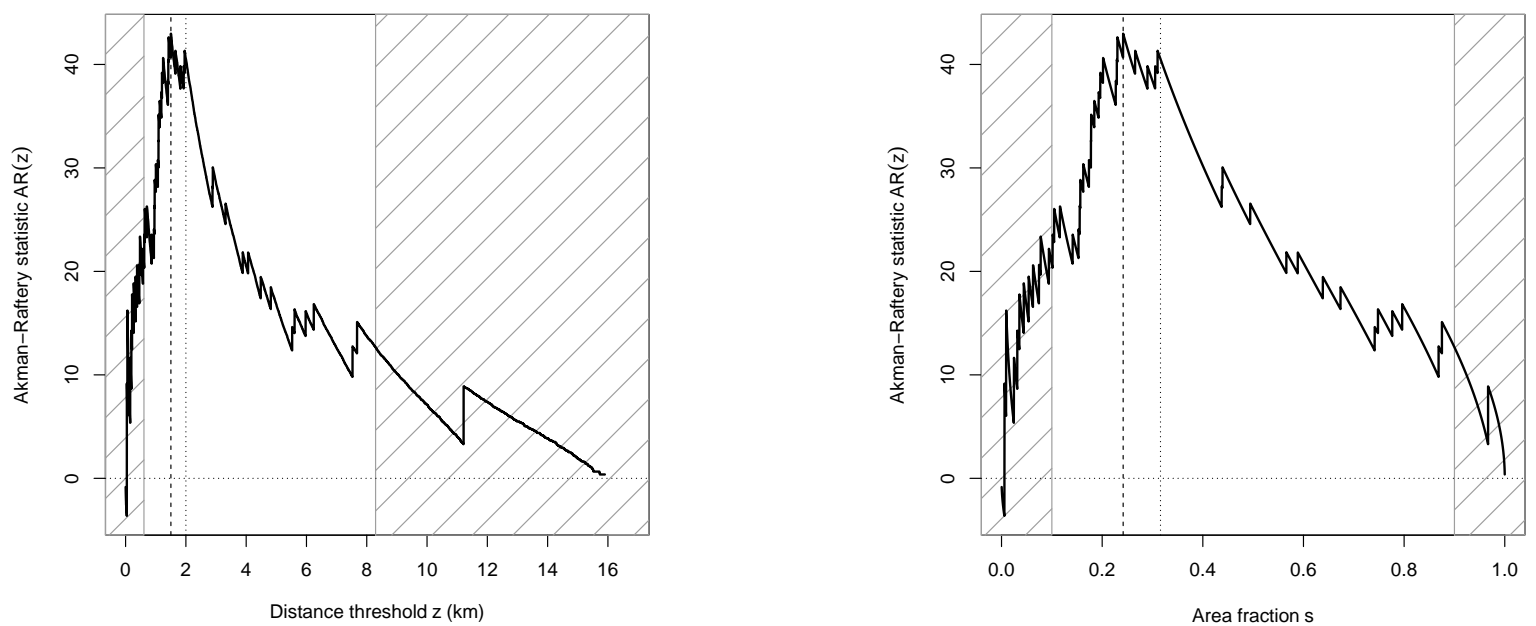

Fig. 14 Akman-Raftery criterion plotted against candidate threshold distance $z$ (Left) and plotted against area fraction $s$ (Right) for the synthetic data of Figure 5 . Dotted vertical line shows correct threshold, $z_{\text {true }}=2 \mathrm{~km}$ (left panel) and corresponding correct area fraction $s_{\text {true }}=0.316$ (right panel). Dashed vertical line shows estimated threshold, $\widehat{z}=1.50 \mathrm{~km}$ (left panel) and corresponding area fraction $\widehat{s}=0.242$ (right panel). Oblique shading covers predictor values below the 10th percentile or above the 90 th percentile.

threshold $z=1.50 \mathrm{~km}$ corresponding to area fraction $s=0.242$; this happens to lie between the 10 th and $90 \mathrm{th}$ percentiles, so that if we had applied the Akman-Raftery constraint that $s$ should lie between $\left[s_{0}, s_{1}\right]=[0.1,0.9]$, the outcome would have been identical. The Akman-Raftery estimated threshold $\widehat{z}=1.50 \mathrm{~km}$ is equal to the estimates obtained by maximising the $\chi^{2}$ statistic or the Studentised contrast, which we described as a mediocre estimate of the true value $2 \mathrm{~km}$.

The theory developed by Akman and Raftery (1986) and Raftery and Akman (1986) has other important advantages, explained in the section SIGNIFICANCE TESTS below.

\section{MATHEMATICAL CONNECTIONS BETWEEN THE CRITERIA}

The threshold selection criteria described above are qualitatively similar to one another; they are all measures of the discrepancy between the observed average densities of deposits above and below the threshold value. Indeed we have been able to prove algebraically that some of them are directly related. The most striking relationship, which we prove in the appendix Connections between the Threshold Criteria, is that

$$
\operatorname{AR}(z)=\sqrt{n} X(z)
$$

That is, the Akman-Raftery criterion $\operatorname{AR}(z)$ defined in (13) is proportional to the signed $\chi^{2}$ test statistic $X(z)$ defined in (7). This means that maximising the Akman-Raftery criterion $\operatorname{AR}(z)$ over all possible thresholds, i.e., without constraints, would be equivalent to maximising the $\chi^{2}$ test statistic $X^{2}(z)$. However, the AkmanRaftery method constrains the range of candidate threshold values, so that the Akman-Raftery method is not quite equivalent to maximising $X^{2}(z)$ over all possible thresholds.

The second relationship can be seen directly by comparing (7) and (8):

$$
X(z)=\frac{Y(z)}{\operatorname{se}_{0}(Y(z))}
$$

where

$$
\operatorname{se}_{0}(Y(z))=\sqrt{s(z)(1-s(z)) / n}
$$

is the standard deviation of $Y(z)$ under the null hypothesis that the density of deposits is uniform. That is, the signed $\chi^{2}$ statistic $X(z)$ is a standardised version of the Youden criterion $Y(z)$. See the appendix Connections between the Threshold Selection Criteria, subsection Relation between $\chi^{2}$ statistic and Youden criterion. This is a "standardised" rather than "Studentised" version because the standard error is calculated under the null model. The relationship (15) does not imply redundancy between $X(z)$ and $Y(z)$, but provides some insight. 
Thirdly, there are qualitative similarities. The WofE contrast (2) is analogous to the Youden criterion (8) except for the use of the logarithm in (2). The statistic $t$ in (4) is the Studentised version of the WofE contrast (2) while the signed $\chi^{2}$ statistic $X(z)$ is the standardised version of the Youden criterion (8).

The overall conclusion is that the different methods for threshold selection described here are not independent methodologies, despite their very different origins. In practice, these different methods cannot be relied upon to provide independent assessments of prospectivity.

\section{SIGNIFICANCE TESTS}

Often it is desired to conduct a statistical test of significance of a binary predictor, or a test for the existence of a threshold effect in a numerical predictor variable $Z$.

\section{Significance Test for a Binary Predictor}

First consider the scenario sketched in Figure 1 where a binary predictor is already given, and we wish to test whether the predictor has a statistically significant effect.

The predictor has no effect if the WofE contrast is zero. To test whether the WofE contrast is statistically significantly different from zero, Goodacre et al. (1993, p. 291) used the Studentised contrast

$$
t=\frac{\widehat{C}}{\operatorname{se}(\widehat{C})},
$$

and determined statistical significance by referring the value of $t$ to a standard Normal distribution. For example, at the usual $5 \%$ level of significance, the contrast is declared to be significant if $|t|>1.96$, or equivalently if $|\widehat{C}|>1.96 \mathrm{se}(\widehat{C})$. See also Cheng $(2007$, p. 321$)$.

The null hypothesis is that the predictor has no effect, so that the density of deposits is uniform over the survey area; the densities inside and outside the buffer are equal, so that the true contrast is zero. The alternative hypothesis is the two-sided alternative that the true contrast is not equal to zero.

The test based on the statistic in equation (17) treats large positive and large negative values of $t$ as being statistically significant. Negative values of $t$ (or $\widehat{C}$ ) suggest that the feature $B$ is antithetical to deposits, and that the complementary feature $\bar{B}$ is prospective. Attaching significance to negative values of contrast may be appropriate in some applications; in their study of seismicity, Goodacre et al. (1993, Table 2, p. 295) reported that some rock types had statistically significant negative values of contrast.

However, in prospectivity analysis, features are usually selected for investigation because our domain knowledge suggests they are likely to be prospective. It is then appropriate to perform a "one-sided" or "one-tailed" test in which only large positive values of $t$ are considered significant; the $5 \%$ critical value 1.96 is changed to 1.64 , that is, the contrast is declared significant if $t>1.64$ or equivalently $\widehat{C}>1.64 \operatorname{se}(\widehat{C})$. This is the familiar distinction between "two-tailed" and "one-tailed" tests (Conover 1999, pp. 78-79). The choice of test depends on the purpose, as discussed above. For brevity, we use two-tailed tests unless otherwise stated.

It may be confusing that the test rule uses the critical points of the Normal distribution, while the test statistic is called "Studentised" and is denoted by the symbol $t$, suggesting that we should use the critical points of the Student's $t$ distribution. This would not be correct because the test statistic $t$ in (17) does not have a Student's $t$-distribution. ${ }^{8}$

Goodacre et al. (1993, pp. 291, 295) point out that a test based on $t$ is valid when the total number of deposits $n$ is large, but would be inaccurate if either of the numbers $n_{B}$ or $n_{\bar{B}}$ is "small". For that eventuality they propose an alternative test based on the binomial distribution (Goodacre et al. 1993, pp. 295-296). The total number of deposits $n$ is treated as fixed, and statistical significance is assessed by referring the observed count $n_{B}$ to the binomial distribution with $n$ trials and success probability $s=a_{B} / a$. This procedure is slightly more complicated, as it involves looking up a table or using software to compute the $p$-value. The binomial distribution can be approximated by the Normal distribution when $n s$ and $n(1-s)$ are both greater than 10 (Carlton and Devore 2014 , p. 181), so we believe that the more refined test of Goodacre et al. (1993) is needed only when $n_{B}$ or $n_{\bar{B}}$ is less than 10 .

${ }^{8}$ The technical requirements for the Student's $t$ distribution are not satisfied: in particular the numerator and denominator of (17) are not independent variables. 
A closely-related test which may be more familiar is the $\chi^{2}$ (chi-squared) test of goodness-of-fit, based on the test statistic

$$
X^{2}=\sum \frac{(\text { observed }- \text { expected })^{2}}{\text { expected }}=\frac{\left(n_{B}-e_{B}\right)^{2}}{e_{B}}+\frac{\left(n_{\bar{B}}-e_{\bar{B}}\right)^{2}}{e_{\bar{B}}},
$$

where $e_{B}=(n / a) a_{B}=n\left(a_{B} / a\right)$ and $e_{\bar{B}}=(n / a) a_{\bar{B}}=n\left(a_{\bar{B}} / a\right)$ are the expected counts in $B$ and $\bar{B}$, respectively, when the deposits are randomly distributed with constant density. The test is performed by referring $X^{2}$ to the $\chi^{2}$ distribution with 1 degree of freedom (Hogg and Craig 1970, Section 10.2, pp. 308-316; Kendall and Stuart 1973, Chapter 30; Pearson 1900). A $\chi^{2}$ variable with one degree of freedom is equivalent to the square of a standard Normal variable, so this particular $\chi^{2}$ goodness-of-fit test is equivalent to computing the signed square root $X= \pm \sqrt{X^{2}}$ and referring $X$ to the standard Normal distribution. In order to take the square root of $X^{2}$, we simplify equation (18) as explained in the appendix Connections between the Threshold Selection Criteria, yielding

$$
X^{2}=n \frac{\left(\frac{n_{B}}{n}-\frac{a_{B}}{a}\right)^{2}}{\frac{a_{B}}{a}\left(1-\frac{a_{B}}{a}\right)}
$$

so that

$$
X=\sqrt{n} \frac{\frac{n_{B}}{n}-\frac{a_{B}}{a}}{\sqrt{\frac{a_{B}}{a}\left(1-\frac{a_{B}}{a}\right)}} .
$$

Note that $X$ is a standardised version of the count $n_{B}$. The contrast is declared statistically significant (i.e. different from zero) at the $5 \%$ significance level if $|X|>1.96$. The one-tailed test would declare significance if $X>1.64$.

The $\chi^{2}$ test is also based on large-sample asymptotic theory and suffers from inaccuracy when the sample size (number of deposits) is small. The established rule of thumb is that both $e_{B}$ and $e_{\bar{B}}$ should be at least 5 (Rice 2006, p. 487; Hogg and Craig 1970, p. 309) implying that $n$ should be at least 10.

Statistical theory shows that the test with the best performance in large samples is the Likelihood Ratio Test, based on the statistic

$$
\text { LRTS }=2 \sum \text { observed } \times \ln \left(\frac{\text { observed }}{\text { expected }}\right)=2\left(n_{B} \ln \frac{n_{B}}{a_{B}}+n_{\bar{B}} \ln \frac{n_{\bar{B}}}{a_{\bar{B}}}-n \ln \frac{n}{a}\right),
$$

which is referred to the $\chi^{2}$ distribution with 1 degree of freedom, so that the estimated contrast $\widehat{C}$ is declared significant at the $5 \%$ level if LRTS $>1.96^{2}=3.84$ (Kendall and Stuart 1973, eq. (30.6), p. 438). The Likelihood Ratio Test also depends on asymptotics but performs well, even in small samples (small numbers of deposits).

For the WofE contrast method for estimating thresholds, the Studentised contrast statistic $(17)$ is $t=1.66 / 0.32=$ 5.19 , which exceeds the $5 \%$ critical value 1.96 for the Normal distribution for the two-sided test. For the $\chi^{2}$ test, the signed square root statistic (20) is

$$
X=\sqrt{48} \frac{34 / 48-1294 / 4102}{\sqrt{(1294 / 4102)(1-1294 / 4102)}}=5.86,
$$

which exceeds 1.96. The Likelihood Ratio Test statistic (21) is

$$
\mathrm{LRTS}=2(34 \ln (34 / 1294)+14 \ln (14 / 2808)-48 \ln (48 / 4102))=31.12,
$$

which far exceeds the $5 \%$ critical value 3.84 for the $\chi^{2}$ distribution with 1 degree of freedom. Such agreement between the outcomes of different hypothesis tests is expected in large samples (large numbers of deposits).

All the significance tests described above perform well, and give similar outcomes, for the synthetic data set. More exotic tests, which have even better small-sample performance, are also available (Read and Cressie 1988).

In summary, the customary test based on the Studentised contrast (17), referred to the standard Normal distribution, is valid provided the total number of deposits $n$ is at least 30 and the expected numbers $e_{B}, e_{\bar{B}}$ both exceed 5. Otherwise, one should use the $\chi^{2}$ test based on (20), or the binomial test described by Goodacre et al. (1993, pp. 295-296), or the Likelihood Ratio Test using (21), or the tests described by Read and Cressie (1988). Our overall recommendation is to use the Likelihood Ratio Test.

The $\chi^{2}$ test is widely used in exploration geoscience (Filzmosera et al. 2005; Garrett 1989; Ghannadpour and Hezarkhani 2016) with caveats (Ballantyne and Cornish 1979). 


\section{The Problem of "Peeking"}

An important caveat about statistical significance tests is that the test conditions must be fixed in advance; it is not valid to change the test procedure in the light of the observed data. The statistical significance tests described in the previous subsection, for testing whether a spatial feature $B$ is prospective, are only valid when the feature $B$ is known and fixed in advance. It would not be valid to perform the same test calculation several times with different choices of $B$, to select the most favorable test result, and then to judge statistical significance using the rules described above.

In particular, if we construct a buffer around a fault system, select the optimal buffer distance by maximising the Studentised contrast $t$, and find that $t(\hat{z})$ for this optimised buffer exceeds 1.96 , then this does not imply statistical significance at the $5 \%$ level. The test based on the statistic $t$ assumes that the buffer distance was fixed in advance. Selecting the "optimal" buffer distance using the observed deposit location data would be a form of cheating, equivalent to selecting the best result among several test outcomes. This is more likely to achieve a spuriously significant outcome, that is, the probability of erroneously declaring "significance" is much greater than 0.05, because of the multiple testing ("look elsewhere") effect (Hochberg and Tamhane 1987; Hsu 1996; Shaffer 1995). See Rice (2006, pp. 60, 321-324) and the XKCD cartoon on jellybean statistics (Anonymous 2011).

Colleagues often ask us why this procedure should be condemned as an instance of "multiple" testing, when they have only performed a single hypothesis test calculation, using the optimised threshold value. Consider that, for each candidate threshold $z$, the value of the statistic $t(z)$ could have been translated into a $p$-value (whether we compute it or not) associated with the hypothesis test for threshold $z$; larger values of $t(z)$ correspond to smaller (i.e. more significant) $p$-values; consequently, by choosing the largest value of $t(z)$ we have equivalently chosen the smallest $p$-value (most significant outcome) out of all the possible significance tests available.

This is tantamount to performing many different statistical tests, each associated with a different value of $z$, but applying the standard of statistical significance that is appropriate for a single test. This is likely to produce spurious significant outcomes, since we will have multiple opportunities to reach the standard of significance for a single test.

When the threshold $\widehat{z}$ is estimated from data, the correct critical value for a $5 \%$ significance test based on $t(\widehat{z})$ is much larger than 1.96, and is more complicated to compute, even for large samples (large numbers of deposits).

\section{Kolmogorov-Smirnov Test}

The problem identified above is that we require a test for the existence of a threshold effect, where the threshold level $z$ is not known in advance. The null hypothesis is that there is no threshold effect, prospectivity is uniform across the survey region, and the deposits are homogeneously randomly scattered. The alternative hypothesis is the simple threshold model, in which prospectivity is higher below the threshold than above the threshold, where the true threshold value $z_{\text {true }}$ is unknown and must be estimated from data. Such a test could be deployed to select the predictor variables to be used in the analysis, from amongst a list of candidate predictors.

Effectively this is a test of significance of the disagreement between two curves, that is, a test of agreement or disagreement between cumulative distribution functions.

The most famous test of this kind is the Kolmogorov-Smirnov (K-S) test (Conover 1999, pp. 295-301), which uses a rescaled version of the maximum discrepancy between the curves $F(z)$ and $G(z)$. In our context, we use the one-sided $\mathrm{K}-\mathrm{S}$ test statistic, $K S_{+}$, which is closely related to the Youden criterion $Y$ defined in (9):

$$
K S_{+}=\sqrt{n} Y_{+},
$$

where $n$ is the number of deposits, $Y$ is the Youden criterion, and $Y_{+}$is its positive value, $Y_{+}=Y$ if $Y \geq 0$, and $Y_{+}=0$ if $Y<0$. The $\mathrm{K}-\mathrm{S}$ test declares the discrepancy to be significant if $K S_{+}>c_{n}$, where $c_{n}$ is the critical value of the test, which depends on the total number of deposits $n$. Reinterpreting Table 4.2 of Stephens (1986, p. 105) we get, for the $5 \%$ level of significance,

$$
c_{n} \approx 1.224 /(1+0.12 / \sqrt{ } n+0.11 / n) .
$$

The critical value $c_{n}$ is a slowly-increasing function of the number of deposits $n$, for example $c_{5}=1.14, c_{10}=1.17$, $c_{100}=1.21$ and $c_{\infty}=1.224$. We emphasise that the $\mathrm{K}-\mathrm{S}$ test does not require large numbers of deposits; the test can be performed for small $n$, using the appropriate critical value $c_{n}$.

For the synthetic data example in Figure 5, we have $Y=0.40$ and $n=48$ so that $K S_{+}=\sqrt{48} \times 0.40=2.77$ and the $5 \%$ critical point is $c_{48}=1.20$, so the test declares the discrepancy to be significant at the $5 \%$ level. 
Other tests related to the capture-efficiency curve include the Anderson-Darling and Cramér-Von Mises tests, and the tests of Berman (1986). The Berman tests were motivated by problems in mineral exploration; Berman's $Z_{2}$ test is based on the area under the capture-efficiency curve. These other tests will be investigated further in a subsequent article.

\section{Akman-Raftery (Constrained Chi-Squared) Test}

The method of Akman and Raftery (1986) and Raftery and Akman (1986) also accounts for the effect of searching for the optimal threshold, enabling us to perform a statistical significance test for existence of a change-point.

Akman and Raftery (1986) show that, if $\widehat{z}$ is the estimated change-point location obtained by the AkmanRaftery method with area fractions $s$ constrained to lie between $s_{0}$ and $s_{1}$, then a test of the hypothesis of no change (i.e. equal densities on each side of the change-point) can be performed using the test statistic

$$
d=(1 / \sqrt{n})|\operatorname{AR}(\widehat{z})|,
$$

where $n$ is the number of deposits. The statistic $d$ is the standardised Akman-Raftery criterion maximised subject to the constraint on area fractions. The approximate $p$-value is (Akman and Raftery 1986, p. 1586)

$$
p \approx 2 \sqrt{\frac{2}{\pi}} \exp \left(-d^{2} / 2\right)(\alpha d-\alpha / d+1 / d)
$$

where $\alpha=(1 / 2) \ln \left(s_{1}\left(1-s_{0}\right) /\left(s_{0}\left(1-s_{1}\right)\right)\right)$. For the synthetic data example with the constraints $s_{0}=0.1, s_{1}=0.9$, we get $d=6.2$ and $p=9.5 \times 10^{-8}$ indicating strong evidence of a threshold.

Since we have established in the appendix Connections between the Threshold Selection Criteria that the $\chi^{2}$ test statistic satisfies $X(z)=\operatorname{AR}(z) / \sqrt{n}$, the results of Akman and Raftery give us a test, based on the $\chi^{2}$ test statistic, for the presence of a threshold. One simply replaces $d$ by $X(\widehat{z})$ in $(25)$. That is, the signed square root of the $\chi^{2}$ test statistic, at the selected threshold, has the $p$-value given by (25) with $d$ replaced by $X(\widehat{z})$.

Akman and Raftery (1986, p. 1587) also mention that their $p$-value formula (25) could be used to construct a confidence interval for the true threshold value, by "inversion" of the formula. To do this, we would consider $d(z)=(1 / \sqrt{n})|\operatorname{AR}(z)|$ as a function of $z$, evaluate $p=p(z)$ for each $d=d(z)$ according to (25), plot $p(z)$ against $z$, and find the range of $z$ values where the $p$-values exceed 0.05 . However, (25) is accurate only for large $n$ and large $d$, so this confidence interval is unlikely to be valid when the number of deposits is small.

\section{SIMULATION EXPERIMENTS}

Simulation experiments based on synthetic data were conducted in order to objectively evaluate and compare the performance of the different techniques. This cannot be done with real data, because the true threshold is not known. The simulation experiments involve a simple, ideal scenario where there is a single best threshold in the sense of Bonham-Carter (1995, p. 319). Performance of each technique is measured by its ability to identify this correct threshold.

\section{Experiment 1}

In this experiment we generated 10,000 simulated outcomes of the synthetic model described in subsection Synthetic Example of the section on THE THRESHOLDING PROBLEM. The prospective region is the buffer zone shown in the left panel of Figure 5. Deposit locations were generated randomly with an average density of 0.02 and 0.01 points per $\mathrm{km}^{2}$ inside and outside the buffer, respectively. The right panel of Figure 5 shows a typical simulated outcome. The correct threshold distance is $z_{\text {true }}=2 \mathrm{~km}$. The prospective region $B$ within distance 2 $\mathrm{km}$ has area $a_{B}=1294 \mathrm{~km}^{2}$, and its complement has area $a_{\bar{B}}=2809 \mathrm{~km}^{2}$. The expected number of deposits is $0.02 \times 1294=25.9$ inside $B$ and $0.01 \times 2809=28.1$ outside $B$, making an expected total of 54.0 deposits.

For each simulated outcome, the threshold distance $z$ was estimated by maximising the estimated contrast, the Studentised contrast, the log-likelihood, the Akman-Raftery criterion with constraints, the Youden criterion and the $\chi^{2}$ test statistic. We denote these methods by C, t, LL, ARM, Y, and X2, respectively.

For the Akman-Raftery method (ARM), following their recommendation, we set $s_{0}=0.1, s_{1}=0.9$ so that solutions were constrained to lie between the first and ninth deciles of the predictor (i.e. of the predictor values at all locations in the study region), which are $0.609 \mathrm{~km}$ and $8.290 \mathrm{~km}$ respectively. This constraint prevents the 
algorithm from choosing extreme values of the threshold, and confers a somewhat unfair or unrealistic advantage on the Akman-Raftery method in our comparison experiment.

For each estimation method, the spread of 10,000 threshold values $\widehat{z}$ obtained by applying the method to the simulated patterns could be portrayed as a histogram. To simplify the results, we have used kernel density estimation, effectively smoothing the histogram. The left column of Figure 15 shows probability densities of the threshold values obtained from each estimation method. Probability densities were computed by Gaussian kernel density estimation (Silverman 1986; Wand and Jones 1995) with bandwidth selected by Silverman's rule (Silverman 1986, p. 48, eq. (3.31)), both implemented in the function density.default in the R system (R Development Core Team 2018).
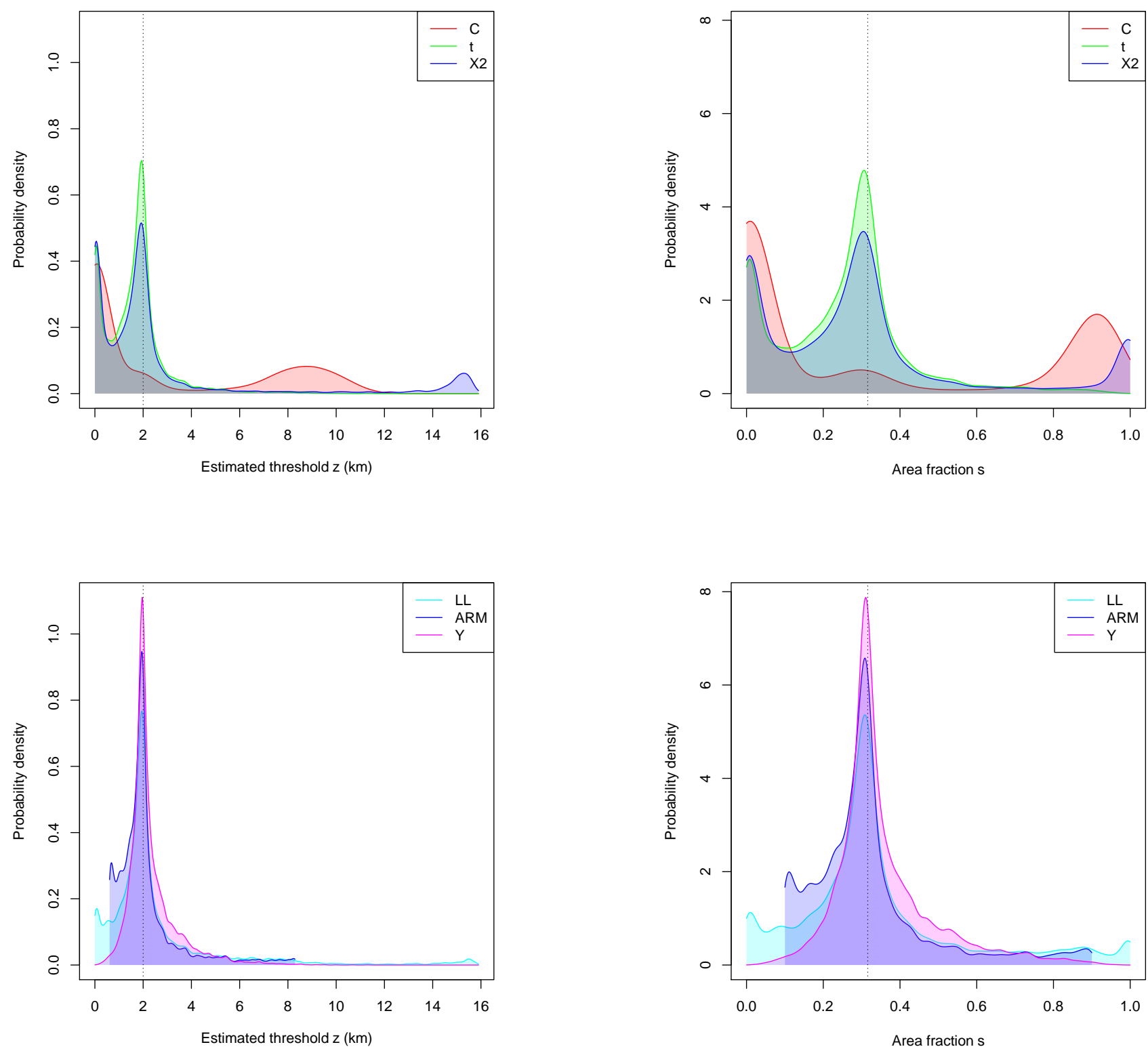

Fig. 15 Performance of methods for selecting the threshold in Experiment 1. Kernel density estimates of the estimated threshold (Left column) and the corresponding buffer area fraction (Right column). In the top row, thresholds were selected by methods C, $\mathrm{t}$, and $\mathrm{X} 2$, which maximise the estimated contrast, Studentised contrast, and the $\chi^{2}$ test statistic, respectively. In the bottom row, thresholds were selected using methods LL, ARM, and Y, which maximise the log-likelihood, the Akman-Raftery criterion with constraints, and the Youden criterion, respectively. Dotted vertical lines indicate the correct threshold, $z_{\text {true }}=2 \mathrm{~km}$ (left column), and corresponding area fraction, 0.32 (right column). 
To avoid misinterpretation, we emphasise that each curve in the left column of Figure 15 is a smoothed histogram of the threshold values selected by a given method applied to the 10,000 simulations. The horizontal axis represents the threshold value that was selected; the vertical axis represents the relative frequency with which this value was selected. While all curves have a peak near the correct threshold value, indicating that the selected thresholds are often close to the correct one, many of the curves also have a steep incline at the left, indicating that they have a substantial chance of erroneously selecting a threshold close to zero.

In the top left panel of Figure 15, the curve for method C does not have a peak around $2 \mathrm{~km}$, indicating that this method is unlikely to produce a threshold value $\widehat{z}$ close to the correct value $z_{\text {true }}=2$. Instead the curve has a steep incline at the left, and a substantial bump around $9 \mathrm{~km}$. This method very often produces a value close to 0 $\mathrm{km}$, and frequently produces values around $9 \mathrm{~km}$, which are both unacceptable. Areas under the density curve are probabilities; the area under the density bump around $9 \mathrm{~km}$ is 0.31 , implying that in about 30\% of all outcomes, method $C$ would have selected a threshold between 6 and $12 \mathrm{~km}$.

The selected threshold values $\widehat{z}$ were also converted to fractions of survey area, $\widehat{a}=a(\widehat{z}) / a$. That is, $\widehat{a}$ is the fraction of survey area deemed to be prospective by the threshold selection procedure. As discussed previously, this transformation eliminates the effect of spatial structure, such as the spatial pattern of faults. Kernel estimates of probability densities of the area fractions $\widehat{a}$ are shown in the right column of Figure 15 .

Table 3 shows objective performance measures for each method of threshold estimation. If the estimation error is $e=\widehat{z}-z_{\text {true }}$, then "bias" is the mean value of $e$, "median deviation" is the median of $e$, "SD" (standard deviation) is the standard deviation of $e$, "MAD" (mean absolute deviation) is the mean of the absolute value of $e$, "RMSE" is the root-mean-square of $e$ (the square root of the mean of $e^{2}$ ), and the last two rows indicate the fraction of outcomes where the absolute value of $e$ exceeded 0.5 and 1 , respectively. In statistical literature, overall performance is often evaluated using RMSE or MAD, while the other indices provide insight into the nature of the errors. Table 4 shows the corresponding performance measures on the scale of the area fractions.

\begin{tabular}{|r|rrrrrr|}
\cline { 2 - 7 } \multicolumn{1}{c|}{} & \multicolumn{7}{c|}{ METHOdS } \\
\hline PERFormance MEASURES & $\mathrm{C}$ & $\mathrm{t}$ & $\mathrm{X} 2$ & $\mathrm{ARM}$ & $\mathrm{LL}$ & $\mathrm{Y}$ \\
\hline BIAS & 0.94 & -0.39 & 0.74 & $\mathbf{0 . 1 3}$ & 0.58 & 0.34 \\
MEDIAN DEVIATION & -1.82 & -0.30 & -0.27 & -0.11 & -0.06 & $\mathbf{0 . 0 3}$ \\
SD & 3.89 & 1.27 & 4.00 & 1.37 & 2.62 & $\mathbf{1 . 0 6}$ \\
MAD & 3.18 & 0.93 & 2.13 & 0.82 & 1.36 & $\mathbf{0 . 6 5}$ \\
RMSE & 4.01 & 1.33 & 4.07 & 2.68 & 2.68 & $\mathbf{1 . 1 1}$ \\
$\mathbb{P}(\mid$ deviation| $>0.5)$ & 0.93 & 0.55 & 0.64 & 0.48 & 0.53 & $\mathbf{0 . 3 8}$ \\
$\mathbb{P}(\mid$ deviation $\mid>1)$ & 0.90 & 0.38 & 0.50 & 0.26 & 0.36 & $\mathbf{0 . 1 9}$ \\
\hline
\end{tabular}

Table 3 Performance of threshold estimation methods in Experiment 1, based on deviation between the estimated and true threshold distances. Boldface numerals indicate the best performance in each row.

\begin{tabular}{|r|rrrrrr|}
\cline { 2 - 7 } \multicolumn{1}{c|}{} & \multicolumn{7}{c|}{ METHODS } \\
\hline PERFormance MEASURES & $\mathrm{C}$ & $\mathrm{t}$ & $\mathrm{X} 2$ & $\mathrm{ARM}$ & $\mathrm{LL}$ & $\mathrm{Y}$ \\
\hline BIAS & 0.008 & -0.070 & -0.012 & $\mathbf{0 . 0 0 4}$ & 0.031 & 0.041 \\
MEDIAN DEVIATION & -0.288 & -0.044 & -0.039 & -0.017 & -0.008 & $\mathbf{0 . 0 0 3}$ \\
$\mathrm{SD}$ & 0.399 & 0.171 & 0.286 & 0.164 & 0.226 & $\mathbf{0 . 1 2 9}$ \\
MAD & 0.361 & 0.136 & 0.205 & 0.108 & 0.151 & $\mathbf{0 . 0 8 6}$ \\
RMSE & 0.399 & 0.185 & 0.286 & 0.164 & 0.229 & $\mathbf{0 . 1 3 6}$ \\
$\mathbb{P}(\mid$ deviation| $>0.05)$ & 0.940 & 0.630 & 0.700 & 0.571 & 0.610 & $\mathbf{0 . 4 8 6}$ \\
$\mathbb{P}(\mid$ deviation $\mid>0.1)$ & 0.913 & 0.477 & 0.577 & 0.383 & 0.451 & $\mathbf{0 . 2 8 1}$ \\
\hline
\end{tabular}

Table 4 Performance of threshold estimation methods in Experiment 1, based on deviation between the estimated and true area fractions of the prospective region. Boldface text indicates best performance in each row.

The most striking result is that method C, based on maximising the WofE contrast, performs very poorly. The methods $t$ and $X 2$, based respectively on the Studentised contrast and the $\chi^{2}$ test statistic, perform much better than C, and frequently produce good estimates of the threshold. However, the steep inclines at the left of each panel in Figure 15 and the large values in the last two rows of Tables 3 and 4 indicate that $t$ and X2 are also liable to produce a value close to zero $\mathrm{km}$. In a single outcome (which a geologist would work with), these methods could well have chosen an unacceptably low value.

Three methods which perform quite well are the profile log-likelihood LL, the Akman-Raftery (constrained chi-squared) method ARM, and the Youden method Y. These methods are very likely to produce a threshold value close to the correct value. 
The Akman-Raftery method ARM includes the restriction that the threshold should lie between the 10th and 90th percentiles of the predictor. The statistical performance of ARM is expected to be better than for X2, and this is borne out by the results in Tables 3 and 4 and Figure 15. The constraint has a substantial effect; ARM and $\mathrm{X} 2$ give different results in $37 \%$ of simulated realisations. The probability density plots in Figure 15 show the results for $\mathrm{X} 2$ in the top row, and for ARM in the bottom row; note the substantial bumps of probability mass near the endpoints for $\mathrm{X} 2$, and the truncation of the curves for ARM.

This simulation experiment is just one instance of an infinite universe of possible spatial patterns of faults. The results in Table 3 and in the left column of Figure 15 depend very specifically on the spatial pattern of faults in Figure 5. Fortunately, this effect is eliminated if we measure performance on the scale of the area fractions, so that the simulation results in Table 4 and in the right column of Figure 15 can be extrapolated to predict performance in a wide range of real situations.

\section{Experiment 2}

Since performance is expected to depend on sample size (i.e. number of deposits), our second experiment is a modification of the first experiment, in which the expected sample size is reduced by a factor of 4 (i.e. each of the density values is reduced by a factor of 4 ). There is now an expected total number of $54 / 4=13.5$ deposits; different simulated datasets will have different numbers of deposits, with an average of 13.5 deposits. The true contrast $C_{\text {true }}$ and true threshold $z_{\text {true }}$ are the same as in Experiment 1.

Figure 16 and Tables 5-6 show the corresponding results. The performance of all methods is degraded by the reduction in the amount of data, as we would expect. The threshold selection methods $C$ and $t$, based respectively on the estimated contrast and the Studentised contrast, both perform substantially worse than they did in Experiment 1, with a much greater propensity to choose small threshold values. The Youden method $Y$ has the best performance, closely followed by ARM, then the profile likelihood method, and then X2. We also found that ARM and $\mathrm{X} 2$ gave different results in $45 \%$ of simulations.

\begin{tabular}{|r|rrrrrr|}
\cline { 2 - 7 } \multicolumn{1}{c|}{} & \multicolumn{7}{c|}{ METHODS } \\
\hline PERFormanCE MEASURES & $\mathrm{C}$ & $\mathrm{t}$ & $\mathrm{X} 2$ & $\mathrm{ARM}$ & $\mathrm{LL}$ & $\mathrm{Y}$ \\
\hline BIAS & $\mathbf{0 . 0 4}$ & -0.43 & 1.38 & 0.65 & 1.23 & 0.79 \\
MEDIAN DEVIATION & -0.92 & -0.75 & -0.39 & -0.13 & $\mathbf{- 0 . 0 6}$ & 0.21 \\
SD & 2.28 & $\mathbf{1 . 5 8}$ & 4.38 & 2.10 & 3.56 & 1.76 \\
MAD & 1.92 & 1.30 & 2.89 & 1.50 & 2.26 & $\mathbf{1 . 2 8}$ \\
RMSE & 2.28 & $\mathbf{1 . 6 4}$ & 4.60 & 2.19 & 3.77 & 1.93 \\
$\mathbb{P}(\mid$ deviation $\mid>0.5)$ & 0.88 & 0.75 & 0.82 & 0.71 & 0.75 & $\mathbf{0 . 6 3}$ \\
$\mathbb{P}(\mid$ deviation $\mid>1)$ & 0.79 & 0.58 & 0.68 & 0.50 & 0.58 & $\mathbf{0 . 4 2}$ \\
\hline
\end{tabular}

Table 5 Performance of threshold estimation methods in Experiment 2 (expected total number of deposits reduced from 54 to 13.5), based on deviation between the estimated and true threshold distances. Boldface text indicates best performance in each row.

\begin{tabular}{|r|rrrrrr|}
\cline { 2 - 7 } \multicolumn{1}{c|}{} & \multicolumn{7}{c|}{ METHODS } \\
\hline PERFormancE MEASURES & $\mathrm{C}$ & $\mathrm{t}$ & $\mathrm{X} 2$ & $\mathrm{ARM}$ & $\mathrm{LL}$ & $\mathrm{Y}$ \\
\hline BIAS & $\mathbf{- 0 . 0 3 1}$ & -0.082 & 0.044 & 0.058 & 0.073 & 0.088 \\
MEDIAN DEVIATION & -0.140 & -0.113 & -0.057 & -0.018 & $\mathbf{- 0 . 0 0 8}$ & 0.029 \\
SD & 0.293 & 0.211 & 0.342 & 0.241 & 0.292 & $\mathbf{0 . 2 0 3}$ \\
MAD & 0.264 & 0.188 & 0.278 & 0.187 & 0.232 & $\mathbf{0 . 1 6 2}$ \\
RMSE & 0.295 & 0.226 & 0.345 & 0.248 & 0.302 & $\mathbf{0 . 2 2 0}$ \\
$\mathbb{P}(\mid$ deviation| $>0.05)$ & 0.910 & 0.810 & 0.861 & 0.784 & 0.811 & $\mathbf{0 . 7 2 1}$ \\
$\mathbb{P}(\mid$ deviation $\mid>0.1)$ & 0.843 & 0.681 & 0.766 & 0.631 & 0.680 & $\mathbf{0 . 5 3 8}$ \\
\hline
\end{tabular}

Table 6 Performance of threshold estimation methods in Experiment 2, based on deviation between the estimated and true area fractions of the prospective region. 

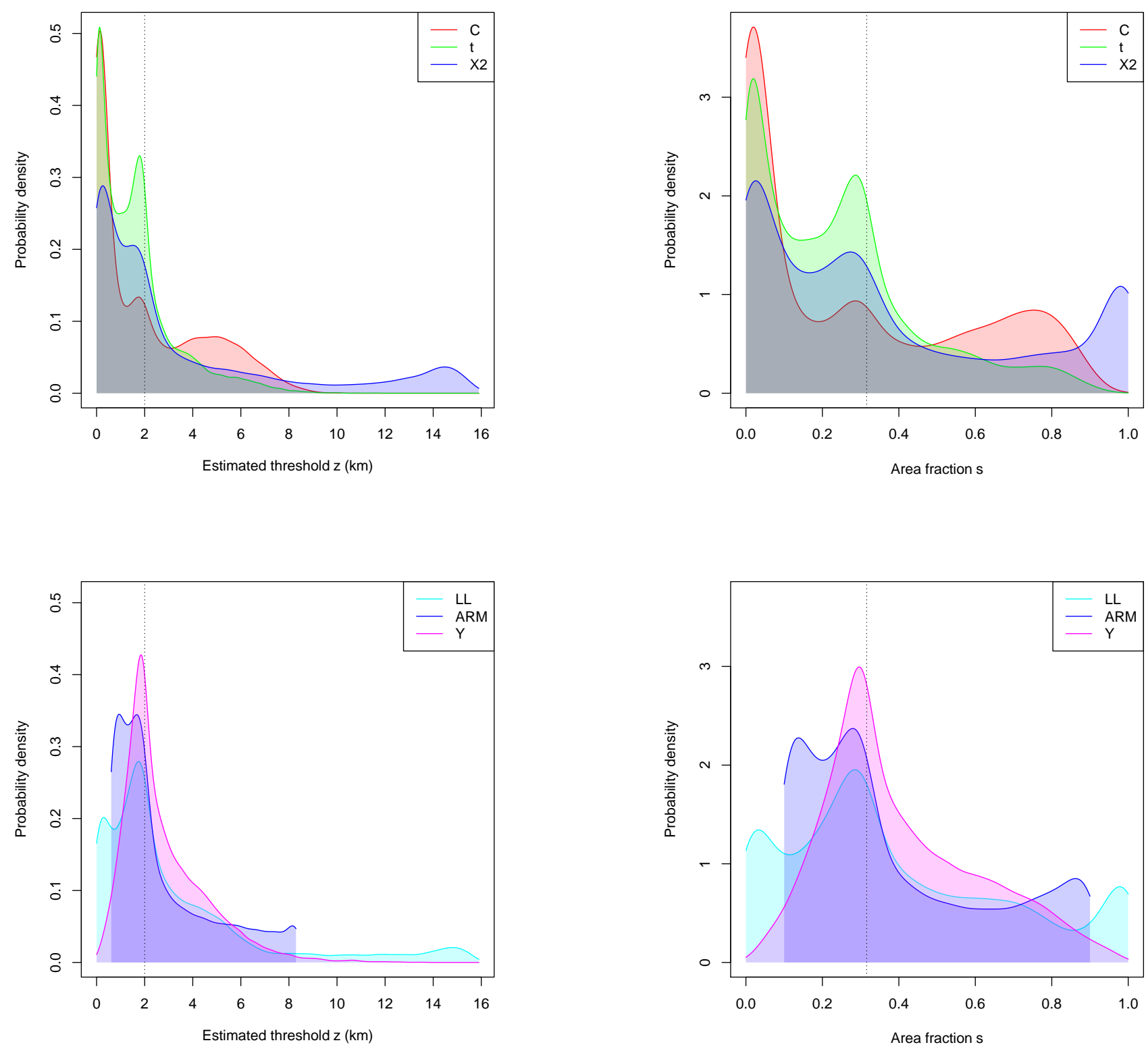

Fig. 16 Experiment 2 counterpart of Figure 15, that is, with the expected total number of deposits reduced from 54 to 13.5. 


\section{ANALYSIS OF MURCHISON GOLD DATA}

\section{Analysis Based on Full Dataset}

Our analysis uses the distance-to-nearest-fault as a predictor. Figure 17 shows the cumulative number of gold deposits plotted against distance from the nearest fault (left panel) and plotted against buffer area (right panel). These are the distribution comparison plots analogous to those in Figure 9, but their behaviour is quite different from those in the synthetic example. The plots in Figure 17 show evidence of three or possibly four segments having distinct slopes, decreasing with increasing distance. Some reasons for such behaviour are discussed in the DISCUSSION section, under What Happens if the Simple Threshold Model Does Not Hold?.

Note that the left panel of Figure 17 has been truncated to a maximum distance of $15 \mathrm{~km}$, which captures $95 \%$ of the deposits but only $40 \%$ of the survey area. This explains why the dashed cumulative curve in the left panel does not reach $100 \%$.

The first outcome of the analysis is that buffer distances larger than $10 \mathrm{~km}$ are largely irrelevant for the purpose of selecting a threshold. This is consistent with the findings of Groves et al. (2000, Fig. 8a, p. 22) that over $95 \%$ of large deposits (with $>1.5 \mathrm{t}$ Au production) in the Kalgoorlie Terrane are located within $5 \mathrm{~km}$ of regional-scale faults, and $95 \%$ of small deposits $(\leq 1.5 \mathrm{t} \mathrm{Au})$ within $10 \mathrm{~km}$ of regional scale faults. Bierlein et al. (2006, Fig. 10b, p. 119) showed that gold endowment falls to a very low level beyond $5-10 \mathrm{~km}$ from major faults in the Yilgarn Craton. Bierlein et al. (2008, Fig. 11, p. 74) found that $90 \%$ of gold production occurs within 10 $\mathrm{km}$ from first, second and third order faults in the north to central Sierra Nevada Foothills (USA) Metamorphic Belt. Brown (2002) found that a statistically significant positive association between deposits and proximity to faults persisted up to $4 \mathrm{~km}$ distance in the Kalgoorlie Terrane. However, the geometry of the faults and shear zones in all of the abovementioned provinces shows that major faults are separated by $20 \mathrm{~km}$ at most, so that a distance-to-nearest-fault greater than $10 \mathrm{~km}$ is achieved only at locations which are remote from the fault system.

It is not clear whether thresholding is appropriate for the Murchison data. Witt et al. (2013, Appendix, Table A1.58) found a gradual decrease in prospectivity with distance from faults, for the Murchison Domain, using different data which are more complete than those shown in Figure 2. Analysis may need to consider the size or endowment of each deposit; whereas very large gold deposits are likely to have been formed in response to large crustal disturbances, very small deposits may behave like "noise" in the analysis (Knox-Robinson and Groves 1997). For example, the spatial resolution of the predictor data may not be fine enough to support useful prediction of very small deposits. Furthermore, spatial patterns of known gold deposits are influenced by the spatially-varying intensity of survey effort. For the purposes of this paper, we set aside such considerations, and simply demonstrate application of the thresholding method, noting when the method provides warnings about its own applicability.
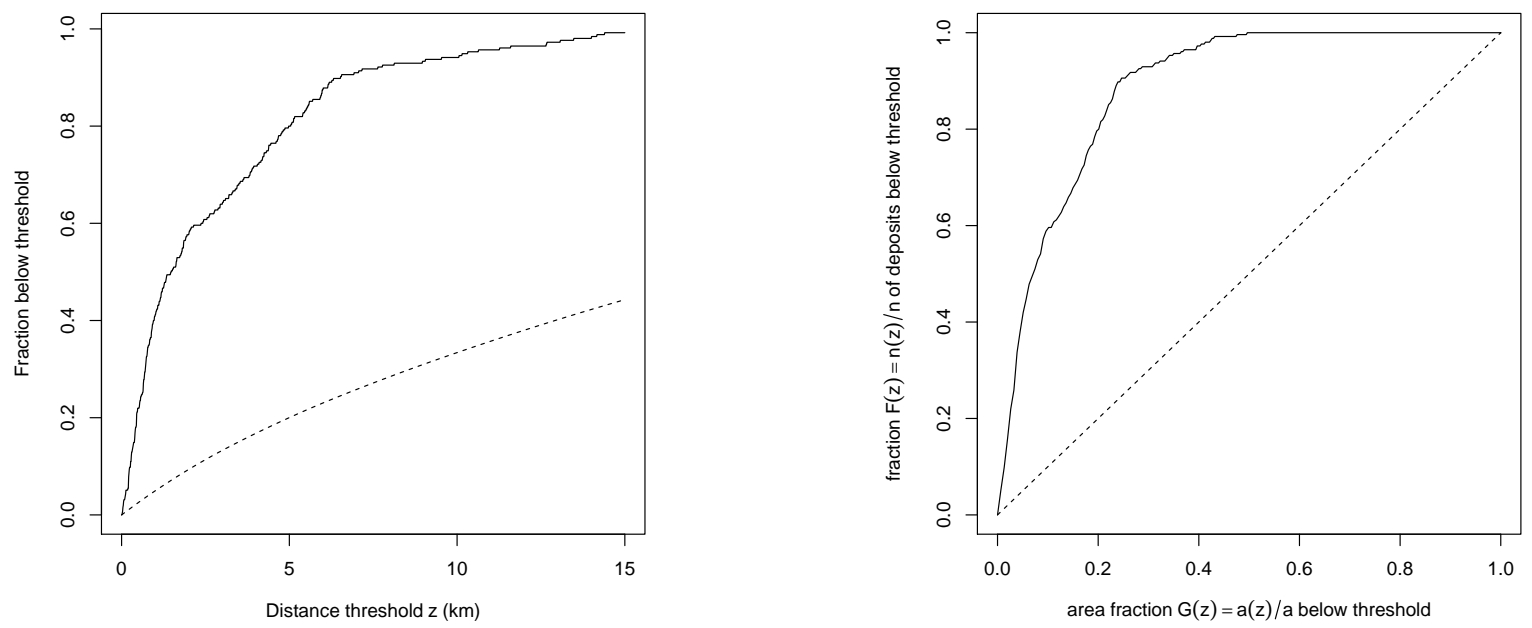

Fig. 17 Counterpart of Figure 9 for the Murchison gold survey data. Left: Cumulative fraction $n(z) / n$ of gold deposits (solid lines) and fraction $a(z) / a$ of survey area (dashed lines) lying within a given distance $z$ of the nearest major fault, plotted against candidate threshold distance z. Right: Capture-efficiency curve; fraction of gold deposits against fraction of survey area, for different threshold distances. 

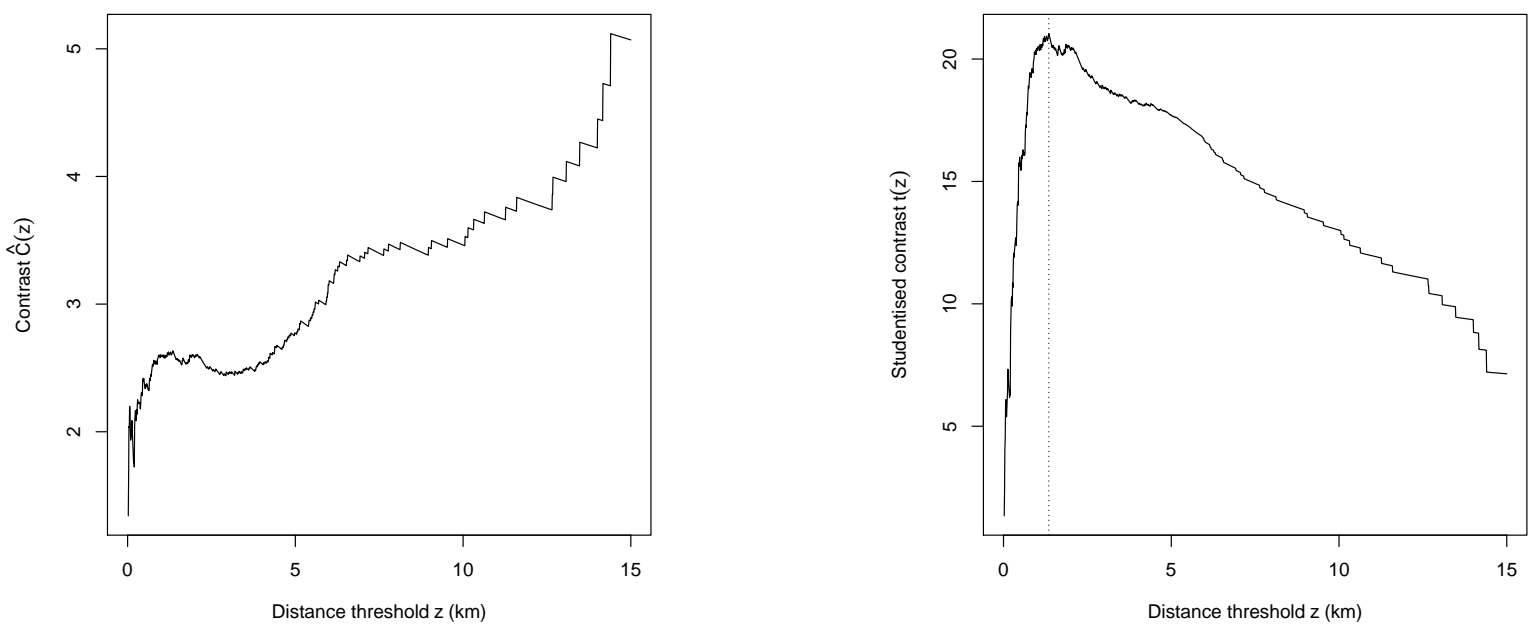

Fig. 18 Weights-of-evidence contrast (Left) and Studentised contrast (Right) plotted against candidate threshold distance $z$ for the Murchison gold data. Vertical line in right panel shows estimated value of threshold, $\widehat{z}=1.35 \mathrm{~km}$.

In Figure 18 the WofE contrast (left panel) and the Studentised contrast (right panel) are plotted against the candidate threshold distance $z$ for the Murchison data. The figure shows erratic behaviour for the WofE contrast, and reasonably stable behaviour with a clear maximum for the Studentised contrast. Again this indicates that the Studentised contrast is to be preferred over the WofE contrast. The estimated threshold using the Studentised contrast is $\widehat{z}=1.35 \mathrm{~km}$.
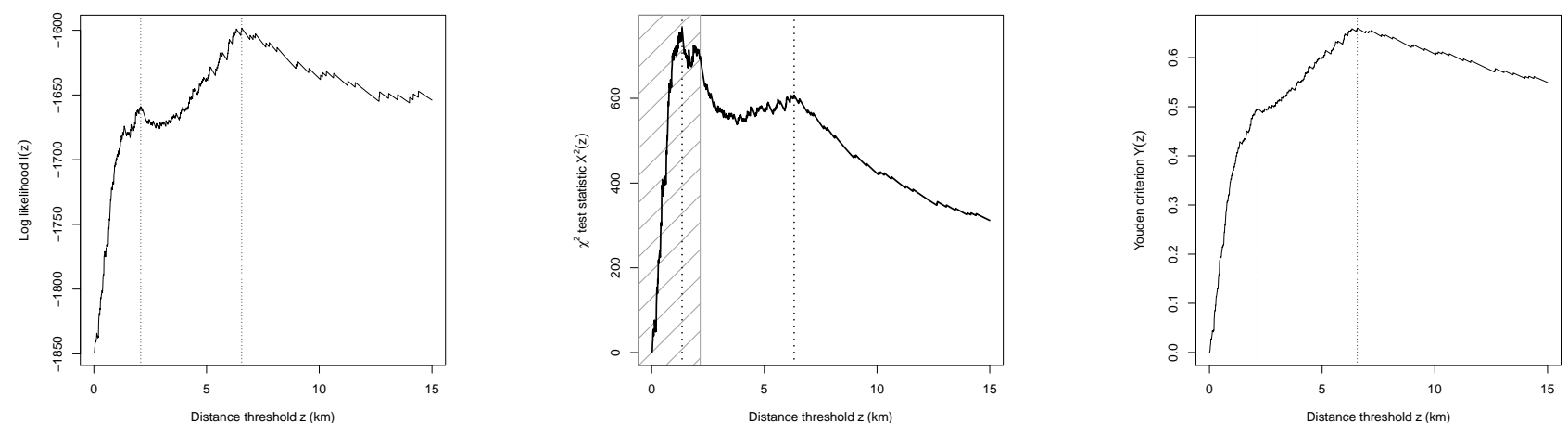

Fig. 19 Profile log-likelihood (Left), $\chi^{2}$ criterion (Middle) and Youden criterion (Right) plotted against candidate threshold distance $z$ for the Murchison gold data. Vertical lines show the estimated threshold and the position of a secondary peak. Shading (Middle) shows the region excluded from consideration in the Akman-Raftery method.

Figure 19 shows the profile log-likelihood (12), $\chi^{2}$ statistic (6) and Youden criterion (8) plotted against the candidate threshold distance $z$, for the Murchison gold survey data. These graphs show evidence of two, or possibly three, local maxima for each criterion. The profile log-likelihood has a maximum at $\widehat{z}=6.56 \mathrm{~km}$ and a secondary peak at $z=2.08 \mathrm{~km}$. The $\chi^{2}$ statistic has a maximum at $\widehat{z}=1.35 \mathrm{~km}$ and a secondary peak at $z=6.32 \mathrm{~km}$. For the Youden method the estimated threshold is $\widehat{z}=6.56 \mathrm{~km}$ and there is a secondary maximum of curvature at about $z=2.15 \mathrm{~km}$. Again, some reasons for such behaviour are considered in the DISCUSSION section, under What Happens if the Simple Threshold Model Does Not Hold?.

In the middle panel of Figure 19 the shading covers the region below the 10th percentile of the distance predictor; the 90 th percentile lies beyond $15 \mathrm{~km}$ and is not shown. The main peak of the $\chi^{2}$ statistic at $\widehat{z}=1.35$ $\mathrm{km}$ lies below the $10 \mathrm{th}$ percentile of distance values $(2.16 \mathrm{~km})$. The Akman-Raftery method ARM does not select this main peak; instead it selects the boundary value $z=2.16 \mathrm{~km}$ which maximises the statistic subject to the constraint. 
The upper limit (90th percentile, $68 \mathrm{~km}$ ) in the Akman-Raftery method is not objectionable, since a distance of more than $20 \mathrm{~km}$ is not geologically plausible (in terms of the understanding of gold mineralization as previously discussed).

Table 7 summarises the results for the Murchison gold data, with $\hat{z}$ indicating the selected distance threshold, and $s(\hat{z})$ the corresponding fraction of survey area.

\begin{tabular}{|r|ll|ll|}
\cline { 2 - 5 } \multicolumn{1}{c|}{} & \multicolumn{2}{c|}{ PRIMARY } & \multicolumn{2}{c|}{ SECONDARY } \\
\hline METHOD & $\hat{z}$ & $s(\hat{z})$ & $\hat{z}$ & $s(\hat{z})$ \\
\hline $\mathrm{t}$ & 1.35 & 0.066 & & \\
$\mathrm{X} 2$ & 1.35 & 0.066 & 6.32 & 0.239 \\
ARM & 2.10 & 0.100 & 6.32 & 0.239 \\
$\mathrm{LL}$ & 6.56 & 0.246 & 2.08 & 0.097 \\
$\mathrm{Y}$ & 6.56 & 0.246 & 2.15 & 0.098 \\
\hline
\end{tabular}

Table 7 Summary of estimates $\hat{z}$ of distance threshold (in km), and corresponding fraction $s(\hat{z})$ of the survey area, using each method applied to the Murchison gold survey data. PRIMARY indicates the estimate obtained by maximising the criterion. SECONDARY is the estimate associated with a smaller peak, if present.

\section{Analysis Based on Restricted Dataset}

An important caveat about WofE, and most other mineral prospectivity techniques, is that the results depend on the survey region: different results can be obtained if the data are restricted to a smaller subset of the survey region.

The left panel of Figure 17 highlights the fact that there are parts of the survey region where the distance-tonearest-fault is far greater than it is for any deposit. It might be reasonable to restrict attention to a subset of the survey region where geological considerations predict that there is a good chance of encountering a gold deposit.

One such calculation which is easy to perform is to restrict attention to a buffer around the fault system, of say 20 kilometres. The preceding formulae are altered only by restricting the distance values $z$ to $0 \leq z \leq 20$, and replacing the area $a$ of the full survey region by the area $a(20)$ of the buffer under consideration. Fractions of survey area $s(z)$ are adjusted by the factor $a / a(20)=1.89$.

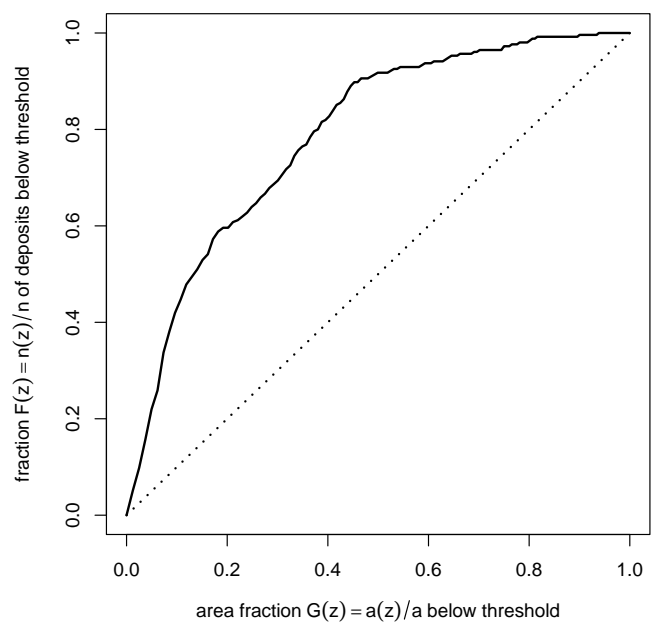

Fig. 20 Capture-efficiency curve for the Murchison gold data within a $20 \mathrm{~km}$ buffer of the fault system.

Figure 20 shows the capture-efficiency curve for the Murchison data in the $20 \mathrm{~km}$ buffer. Figure 21 shows the WofE contrast $\widehat{C}(z)$ and the Studentised contrast $t(z)$ plotted against the candidate threshold distance value $z$. The WofE contrast value is maximised at unrealistically large distances and is not practicable. The Studentised contrast has a clear maximum at about $\widehat{z}=1.35 \mathrm{~km}$.

Figure 22 shows the profile log-likelihood and the Youden criterion plotted against $z$. These both select $\widehat{z}=6.32$ $\mathrm{km}$ as the optimal threshold, with a secondary peak at $\widehat{z} \approx 1.5 \mathrm{~km}$. 

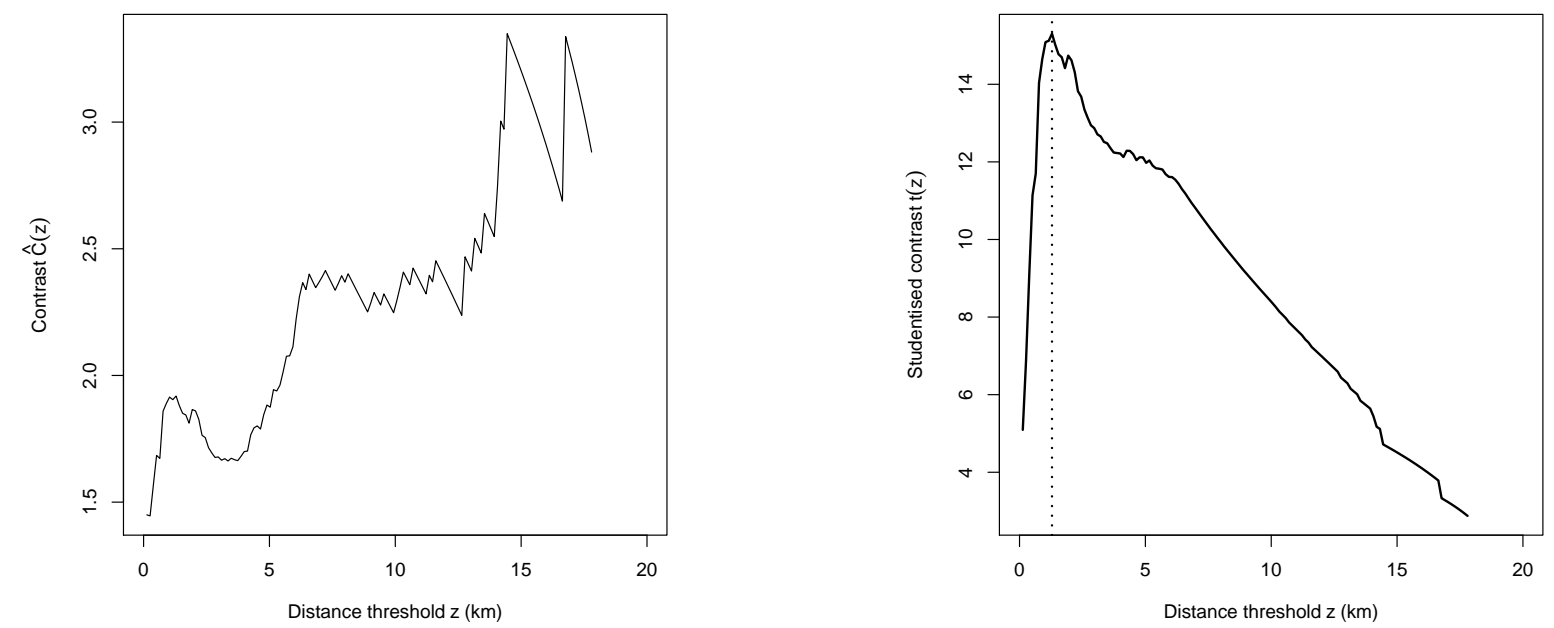

Fig. 21 Weights-of-evidence contrast (Left) and Studentised contrast (Right) plotted against candidate threshold distance $z$, for the Murchison gold data within a $20 \mathrm{~km}$ buffer of the fault system. Vertical line in the right panel shows estimated threshold $\widehat{z}=1.35 \mathrm{~km}$.
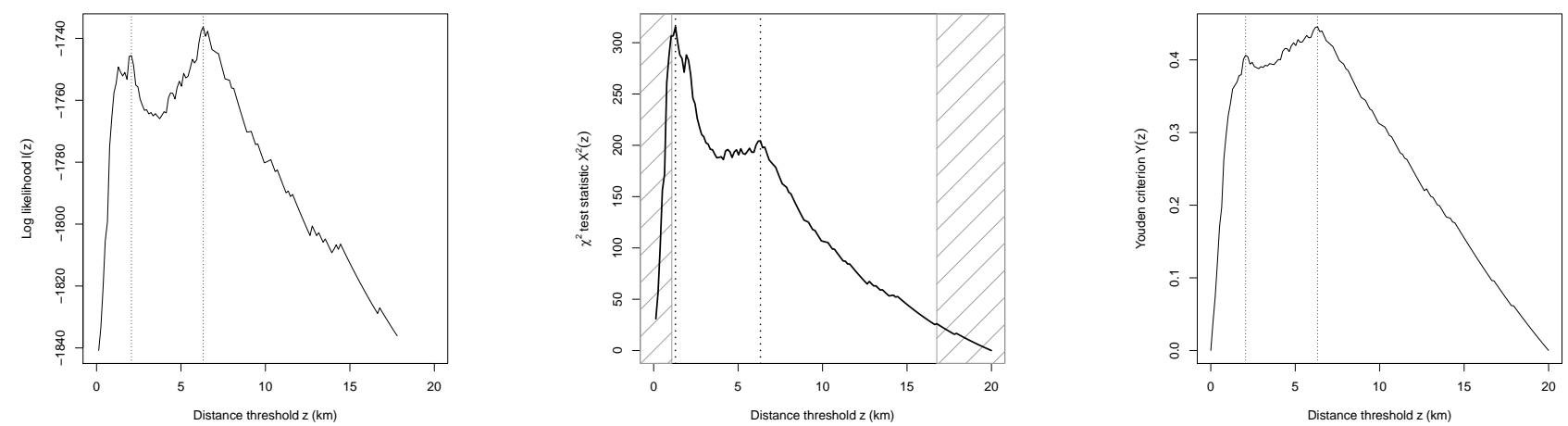

Fig. 22 Profile log-likelihood (Left), $\chi^{2}$ statistic (Middle) and Youden criterion (Right) plotted against candidate threshold distance $z$, for the Murchison gold data within a $20 \mathrm{~km}$ buffer of the fault system. Vertical lines show the selected threshold and a secondary peak. Shading (Middle) shows the region excluded from consideration in the Akman-Raftery method.

\begin{tabular}{|r|ll|ll|}
\cline { 2 - 5 } \multicolumn{1}{c|}{} & \multicolumn{2}{c|}{ PRIMARY } & \multicolumn{2}{c|}{ SECONDARY } \\
\hline METHOD & $\hat{z}$ & $s(\hat{z})$ & $\hat{z}$ & $s(\hat{z})$ \\
\hline $\mathrm{t}$ & 1.29 & 0.119 & & \\
$\mathrm{X} 2$ & 1.29 & 0.119 & 6.32 & 0.452 \\
ARM & 1.29 & 0.119 & 6.32 & 0.452 \\
$\mathrm{LL}$ & 6.32 & 0.452 & 2.06 & 0.182 \\
$\mathrm{Y}$ & 6.32 & 0.452 & 2.06 & 0.182 \\
\hline
\end{tabular}

Table 8 Summary of estimates $\hat{z}$ of distance threshold (in $\mathrm{km}$ ), and corresponding fraction $s(\hat{z})$ of survey area, using each method applied to the Murchison gold survey data within a $20 \mathrm{~km}$ buffer of the fault system.

In this subset of the data, the 10th percentile of distance is $1.07 \mathrm{~km}$, and the $\chi^{2}$ and Akman-Raftery methods are in agreement.

Estimated distance thresholds around $1 \mathrm{~km}$ seem reasonable for regional-scale faults, that is, faults with a strike length of between 10 and $100 \mathrm{~km}$. These distances fall within the range ( 0.8 to $2.4 \mathrm{~km})$ obtained using a variety of methods for crustal- and regional-scale faults in a study of orogenic gold deposits in the Kalgoorlie Terrane by Brown (2002, pp. 248-249 and Table 6.4, p. 252).

Distances of $6 \mathrm{~km}$ are quite high for a regional-scale fault system, but would be consistent with the existence of (a) crustal-scale shear zones (large, complex fault systems consisting of multiple slip surfaces with ductile as well as brittle deformation); or (b) small unmapped local-scale secondary- and tertiary-scale faults associated with the regional faults which control the location of gold deposits; or (c) existence of regional-scale faults which were not detected in the data provided. Both (b) and (c) are plausible in the Yilgarn Craton which includes the Murchison goldfields. 


\section{BOOTSTRAP STANDARD ERRORS AND CONFIDENCE INTERVALS}

The uncertainty (lack of precision) of the estimated threshold is important in practice. Unfortunately, the familiar methods for determining standard errors and confidence intervals cannot be applied to the estimated thresholds calculated in this paper, because those methods are based on the Normal distribution, which is not appropriate here, as explained in the final paragraph of the subsection Profile Likelihood for the Threshold Model in the section THRESHOLD SELECTION USING CHANGE-POINT ANALYSIS.

Bootstrap methods (Efron and Tibshirani 1993) can be used to compute standard errors and confidence intervals for any target quantity. They are particularly useful when the estimator of the target quantity is not Normally distributed. In essence, the variability of estimates of the target quantity is captured by repeating the estimation procedure with many randomised versions of the original data.

In the "nonparametric" bootstrap, the original data are randomly resampled many times; the threshold estimation procedure is applied to these randomised data sets; and the spread of the resulting threshold estimates is used to calculate the standard error or confidence interval. In the "parametric" bootstrap, the simple threshold model is assumed to be true; simulated data are generated from the simple threshold model; the threshold estimation procedure is applied to these simulated data sets, and the resulting threshold estimates are used to calculate the standard error or confidence interval.

\section{Nonparametric bootstrap}

1. Record the predictor values $z_{i}=Z\left(x_{i}\right)$ at the deposit locations $x_{1}, \ldots, x_{n}$.

2. Repeat the following procedure $K$ times:

(a) Generate a random dataset $z_{1}^{\dagger}, \ldots, z_{n}^{\dagger}$ of the same size as the original data, by "resampling" from the original data, that is by selecting a random sample of size $n$ with replacement from amongst the values $z_{1}, \ldots, z_{n}$.

(b) Apply the threshold estimator to the random dataset, yielding an estimate of the threshold, $\widehat{z}$.

3. Collect the $K$ threshold estimates $\widehat{z}^{(1)}, \ldots, \widehat{z}^{(K)}$ computed by the previous step.

4. For the bootstrap standard error, compute the empirical standard deviation of the $\widehat{z}$ values,

$$
\mathrm{SE}=\frac{1}{K-1} \sum_{k=1}^{K}\left(\widehat{z}^{(k)}-\overline{\widehat{z}}\right)^{2}
$$

where $\overline{\widehat{z}}=\frac{1}{K} \sum_{k=1}^{K} \widehat{z}^{(k)}$ is the average estimated threshold. For a bootstrap $95 \%$ confidence interval, compute quantiles of the $\widehat{z}$ values as described below.

The number of repetitions $K$ depends on computer resources, but should typically be at least 10000 .

Since the distribution of estimates $\widehat{z}^{(k)}$ is expected to be asymmetrical, we recommend computing an asymmetric confidence interval. The upper endpoint of this interval is computed as the 95th percentile of the bootstrap values $\widehat{z}^{(k)}$ greater than the data estimate $\widehat{z}$, and the lower endpoint is the 5th percentile of the values $\widehat{z}^{(k)}$ smaller than the data estimate $\widehat{z}$, yielding a $95 \%$ confidence interval.

\section{Parametric bootstrap}

1. Estimate the threshold $z$ from the data.

2. Compute the corresponding area fraction $s=a(z) / a$ below the threshold and record the number $m=$ $n(z)$ of deposits below the threshold.

3. Repeat the following procedure $K$ times:

(a) Generate $m$ random numbers distributed uniformly between 0 and $s$, and $n-m$ random numbers distributed uniformly between $s$ and 1 , yielding $n$ random numbers $s_{1}^{\dagger}, \ldots, s_{n}^{\dagger}$.

(b) Back-transform the area fractions $s_{i}^{\dagger}$ to predictor values $z_{i}^{\dagger}$ using the graph of $G(z)$ (for example the thin solid curve in the left panel of Figure 9). This yields a random dataset of predictor values $z_{1}^{\dagger}, \ldots, z_{n}^{\dagger}$ at the deposits.

(c) Apply the threshold estimator to the random dataset $z_{1}^{\dagger}, \ldots, z_{n}^{\dagger}$ yielding an estimate of the threshold, $\widehat{z}$.

4. For the bootstrap standard error, compute the empirical standard deviation of the $\widehat{z}$ values. For a bootstrap CI, compute quantiles of the $\widehat{z}$ values, as described above for the nonparametric bootstrap.

The parametric bootstrap is typically more accurate, provided the assumed model (in this case the simple threshold model) is true. The nonparametric bootstrap is more robust against violation of the model assumptions. Furthermore, the standard errors will provide a good summary of variability only if the distribution of the $\widehat{z}$ 
values is reasonably symmetric. A pragmatic approach is to inspect the capture-efficiency curve to assess whether it follows the inverted "V" shape expected for the simple threshold model, shown in Figure 10. If so, then the parametric bootstrap can be used; if not, then the nonparametric bootstrap should be used. After calculation, any glaring discrepancy between the parametric and nonparametric bootstrap results would suggest that the simple threshold model does not hold.

We applied the two bootstrap techniques to our example datasets, using 10,000 simulations for each calculation, and using the Youden criterion to select the threshold. Table 9 shows the estimates, bootstrap standard errors and confidence intervals.

\begin{tabular}{|c|c|c|c|c|c|c|c|c|}
\hline \multirow[t]{3}{*}{ DATASET } & \multicolumn{4}{|c|}{ THRESHOLD $(\mathrm{km})$} & \multicolumn{4}{|c|}{ AREA FRACTION (\%) } \\
\hline & \multirow{2}{*}{$\begin{array}{c}\text { Estimate } \\
\hat{z}\end{array}$} & \multicolumn{3}{|c|}{ Bootstrap } & \multirow{2}{*}{$\begin{array}{c}\text { Estimate } \\
s(\hat{z})\end{array}$} & \multicolumn{3}{|c|}{ Bootstrap } \\
\hline & & Method & $\mathrm{SE}$ & CI & & Method & $\mathrm{SE}$ & CI \\
\hline \multirow[t]{2}{*}{ synthetic } & 1.96 & non & 0.26 & $1.27-2.37$ & \multirow[t]{2}{*}{31.6} & non & 3.9 & $20.7-36.8$ \\
\hline & & par & 0.23 & $1.58-2.70$ & & par & 3.2 & $25.6-41.3$ \\
\hline \multirow[t]{2}{*}{ Murchison } & 6.56 & non & 0.41 & $6.00-7.76$ & \multirow[t]{2}{*}{24.6} & non & 1.0 & $23.0-27.8$ \\
\hline & & par & 0.07 & $6.41-6.77$ & & par & 0.2 & $24.2-25.2$ \\
\hline \multirow[t]{2}{*}{ Murchison $(<20 \mathrm{~km})$} & 6.32 & non & 1.15 & $2.06-6.61$ & \multirow[t]{2}{*}{45.2} & non & 7.0 & $18.2-46.8$ \\
\hline & & par & 1.12 & $5.99-6.44$ & & par & 0.6 & $43.5-45.8$ \\
\hline
\end{tabular}

Table 9 Bootstrap standard errors and bootstrap 95\% confidence intervals ( non = nonparametric, par = parametric) calculated for the example datasets using the Youden method to estimate the threshold.

For the synthetic data in the right panel of Figure 5, there is close agreement between the results from the nonparametric and parametric bootstrap methods. This is expected because the synthetic data were created according to the simple threshold model.

For the Murchison gold survey data, the parametric bootstrap gives a smaller standard error and narrower confidence interval than the nonparametric bootstrap. Since the capture-efficiency curve suggests non-conformity to the simple threshold model, the parametric results may be discarded as over-optimistic. Similar comments apply to the restricted Murchison data.

\section{DISCUSSION}

\section{What Happens if the Simple Threshold Model Does Not Hold?}

The methods of threshold selection described in this paper all assume a "simple threshold model", in which density of gold deposits is constant inside a certain region, and constant outside the region, as discussed in the section on THRESHOLD SELECTION USING THE CAPTURE-EFFICIENCY CURVE, subsection Relation to Threshold. Clearly, the Murchison data do not conform to this simple model (Baddeley 2018) and it is important to understand how the different techniques will respond.

Figure 23 sketches three different kinds of relationship between the predictor and prospectivity. ${ }^{9}$ The left column shows the simple threshold model; the middle column is an alternative "plateau" model in which prospectivity is highest when the predictor has a middling value; and the right column is a model in which prospectivity declines gradually as the predictor value increases. The top row depicts the relationship between the predictor value (on the horizontal axis) and the prospectivity (on the vertical axis). The bottom row is the corresponding capture-efficiency curve relating the cumulative fraction of deposits to the cumulative fraction of survey area.

The "plateau" model involves two threshold positions, and the prospectivity is highest when the predictor value falls between these thresholds. This might apply when the predictor is a mechanical property such as the rheological strength of a host rock. Rocks with intermediate strength may allow some brittle fracturing, facilitating focused fluid flow, which is favourable for the formation of orogenic gold deposits. However, very weak rocks with extensive fracturing may allow fluids to disperse without focused fluid flow, while very strong rocks may not fracture at all.

For predictors which represent angles, there cannot be a single threshold, for mathematical reasons. Two cuts are needed to divide a circle into pieces, so the simplest non-trivial model has two thresholds or breakpoints. The strike angles of faults and shear zones are examples of predictor variables where at least two thresholds may apply. Faults and shear zones within a particular range of orientations may be favorable for gold deposit formation (for

${ }^{9}$ For a given value $z$ of the predictor, the prospectivity $\rho(z)$ is defined as the expected density of deposits per unit area amongst those grid cells with predictor value equal to $z$. 
example because faults formed, or preferentially reactivated, in different epochs may be distinguished by their different orientations). Faults and shear zones that are favourably oriented to the regional-scale stress field may be reactivated to produce dilational zones, resulting in fluid influx and gold deposition (Groves et al. 2000). At a district scale (ca. $10 \mathrm{~km}$ ), gold deposits are often located near jogs (deviations of $\sim 10^{\circ}$ to $20^{\circ}$ ) in the overall trend of crustal-scale faults (Groves et al. 2000).

Change-point problems involving several change-points can easily be handled using maximum likelihood, but the other methods (Youden, Akman-Raftery and Studentised contrast) do not easily adapt to this setting. Techniques for applying maximum likelihood with multiple change-points include nonparametric maximum likelihood (Sager 1982) and CART (Breiman et al. 1984).

Multiple thresholds of a single predictor variable are discussed by J. Liu and Cheng (2019) in the context of hydrothermal Au deposits. Polykretis and Chalkias (2018, p. 258) deal with categorical predictors with many possible levels (e.g. rock type variable with many possible types) and propose merging the levels by grouping them according to the sign of the estimated contrast, before applying WofE.

\section{Gradual Decline in Prospectivity}

The right-hand column of Figure 23 sketches a gradual decline in prospectivity with increasing value of the predictor.

One could argue that, in this situation, the data should not be subjected to thresholding, and WofE should not be applied; instead, a more general version of logistic regression should be applied. Here we simply consider what would happen if thresholding were inappropriately applied to such data.

Assume that the density of deposits per unit area is a decreasing function $\rho(z)$ of the predictor value $z$. That is, in grid cells with predictor value $z$, the density of deposits is $\rho(z)$ deposits per unit area, where $\rho(z)$ decreases as $z$ increases. With some algebra (given in the appendix Derivation of Optimal Threshold for Gradual Decline in Prospectivity) it can be shown that the optimal threshold for the Youden method is the threshold $\widehat{z}$ at which $\rho(\widehat{z})$ is equal to the average density of deposits over the study region.
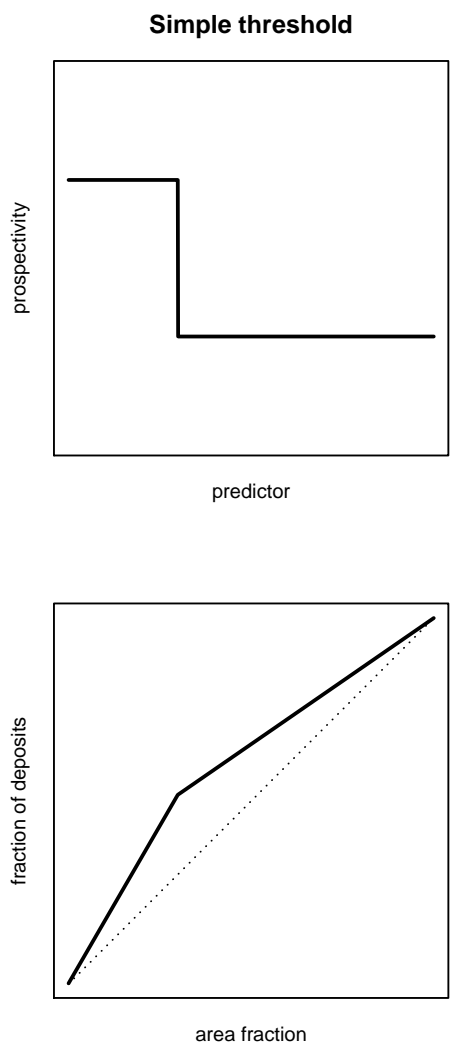
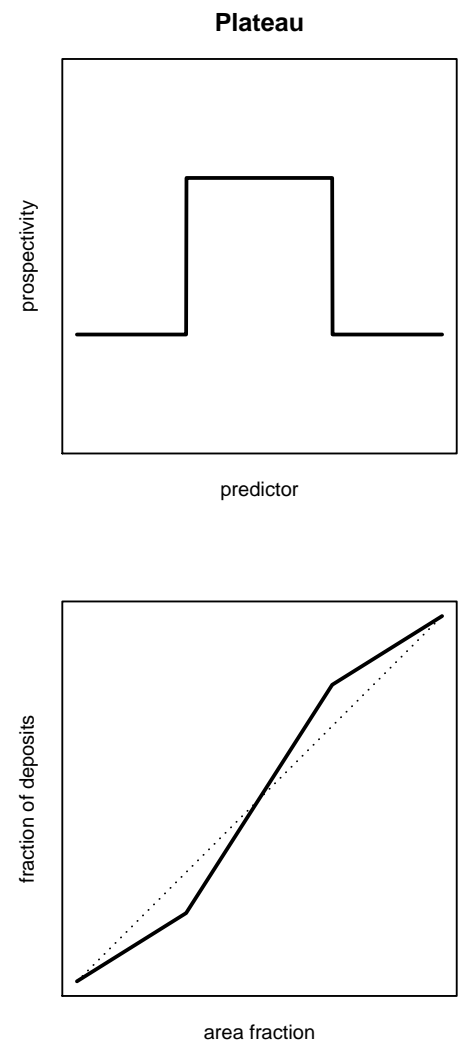
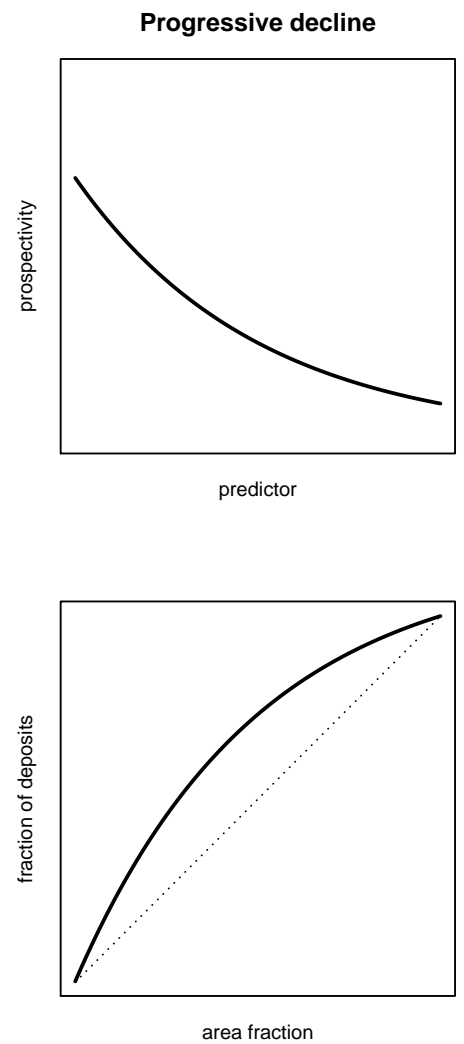

Fig. 23 Three different types of relationship between the predictor and prospectivity. Top row: prospectivity as a function of predictor value. Bottom row: capture-efficiency curve. Left column: simple threshold model. Middle column: plateau model. Right column: gradual decline in prospectivity. 

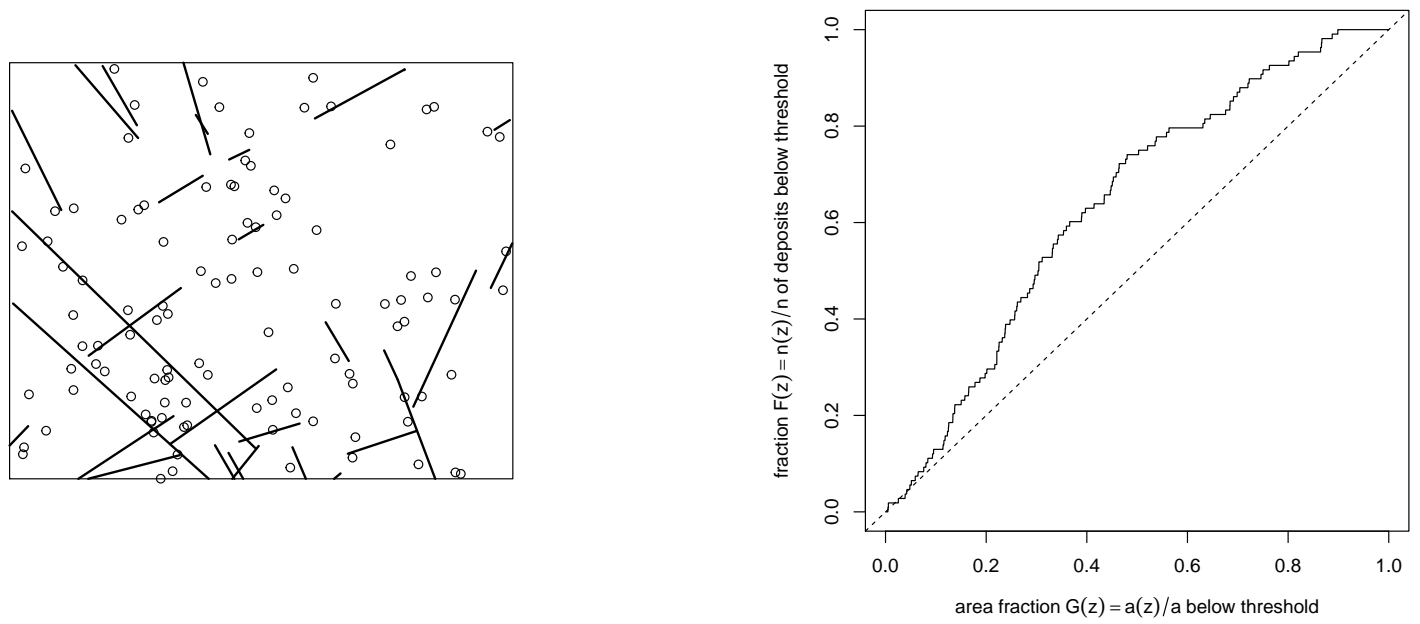

Fig. 24 Synthetic example exhibiting gradual decline in prospectivity with distance. Left: faults and simulated deposit locations. Right: capture-efficiency curve.

Figure 24 shows a synthetic example of gradually-declining prospectivity. It is based on the same spatial pattern of faults as the synthetic example in Figure 5, and the predictor variable $Z$ is once again taken to be the distance to the fault pattern. However in this new example, the density of deposits declines exponentially with distance $z$, in the form $\rho(z)=b e^{-c z}$, where $b$ is the constant density that would give an average of 200 deposits throughout the study region, and $c=1 / 5$. This is equivalent to a logistic regression model (Baddeley 2018). The left panel of Figure 24 shows the fault pattern and the simulated deposit locations. The right panel shows the capture-efficiency curve.

The left panel of Figure 25 shows the WofE contrast $\widehat{C}(z)$ plotted against $z$. The threshold which maximises the contrast is $7.95 \mathrm{~km}$. The contrast is generally increasing with the threshold value, which does not seem plausible, and the obtained threshold does not seem appropriate.

The right panel of Figure 25 shows the Studentised contrast $t(z)$ plotted against $z$. The threshold which maximises the Studentised contrast is $3.21 \mathrm{~km}$.
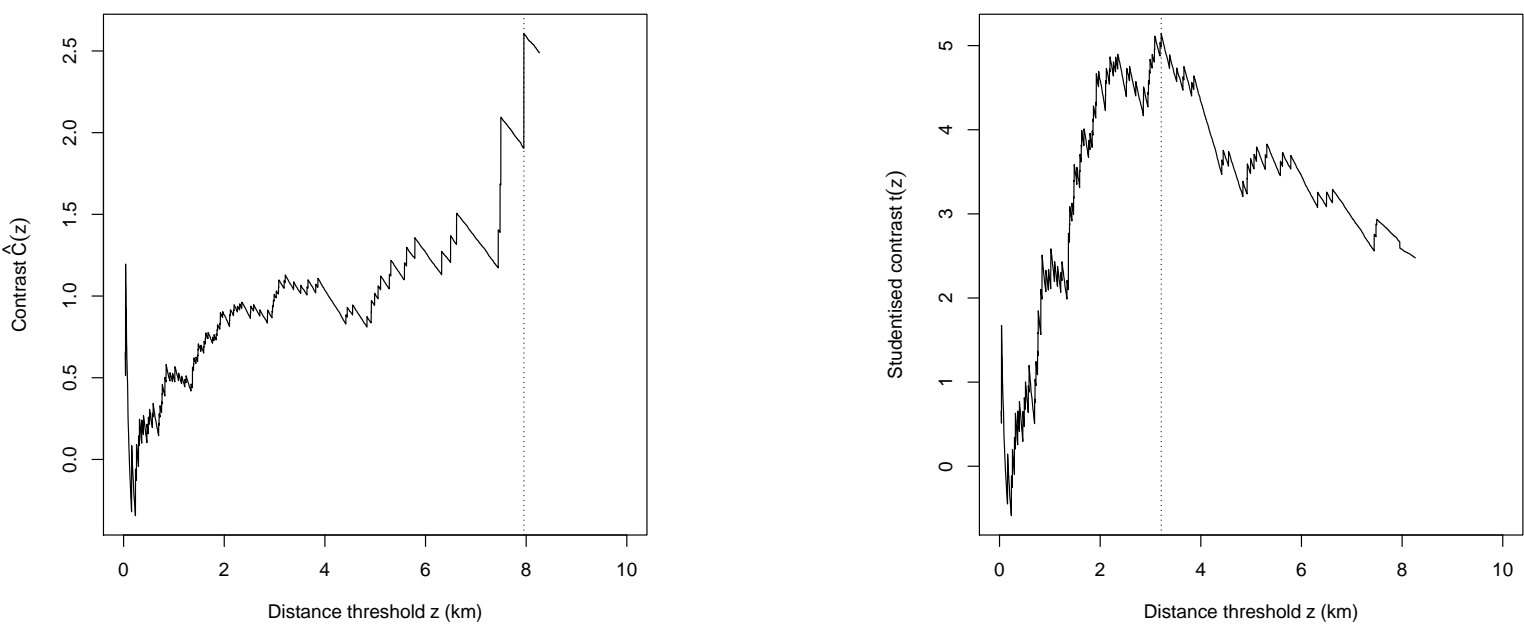

Fig. 25 Weights-of-evidence contrast (Left) and Studentised contrast (Right) for the synthetic example in Figure 24. 

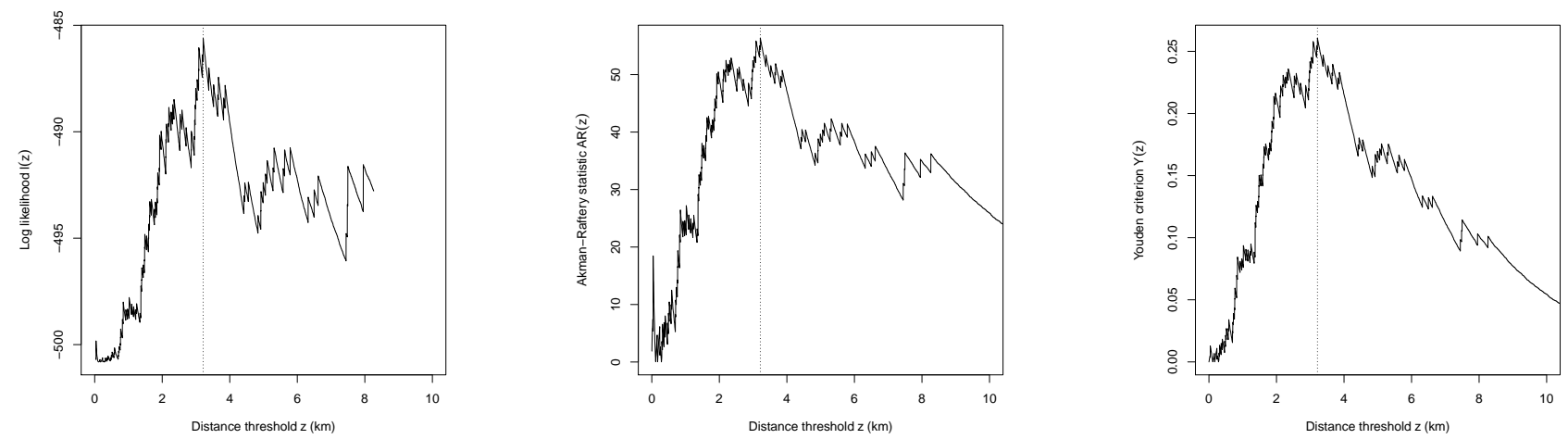

Fig. 26 Profile log-likelihood (Left), Akman-Raftery criterion (Middle) and Youden criterion (Right) for the synthetic example in Figure 24.

The simulated pattern has 108 points, giving an average density of $\widehat{\mu}=108 / 4102=0.0263$ deposits per square kilometre. Solving the equation $\rho(z)=\widehat{\mu}$ gives a predicted optimal threshold of $z=3.08 \mathrm{~km}$, reasonably close to the value obtained by the Youden method, the profile log-likelihood and the Akman-Raftery method.
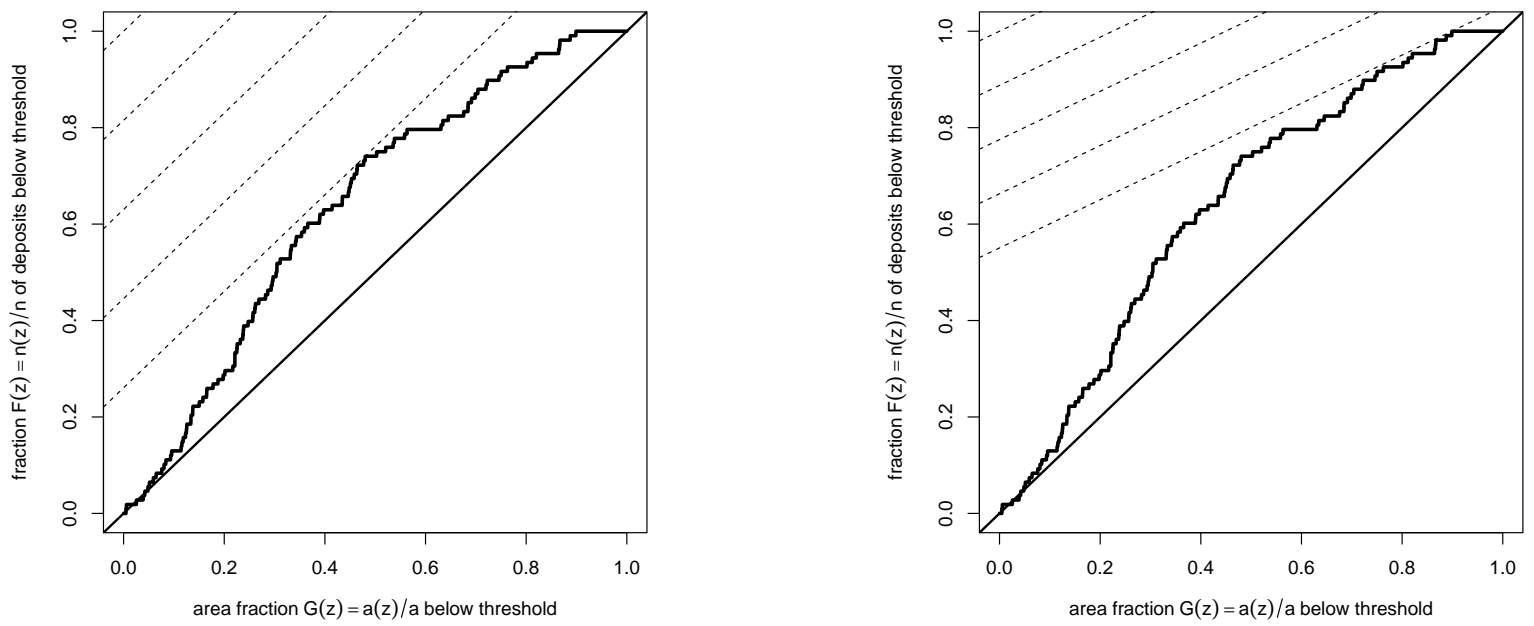

Fig. 27 Effect of cost-benefit ratio on selection of threshold for the synthetic example in Figure 24. Dashed lines have slope equal to cost-benefit ratio. Left: cost-benefit ratio equals 1 . Selected threshold is $3.06 \mathrm{~km}$. Right cost-benefit ratio equals 0.5 . Selected threshold is $9.32 \mathrm{~km}$.

When prospectivity declines gradually as in this example, the cost-benefit ratio (exploration cost to exploitation benefit) becomes more important. The left panel of Figure 27 shows the application of the graphical procedure for maximising the Youden criterion using parallel lines of slope 1. The right panel shows the same procedure using parallel lines of slope $1 / 2$, (i.e. assuming that the value of $1 \%$ of mineral deposits is equal to the cost of exploring $2 \%$ of survey area), which selects a different threshold of $z=8.26 \mathrm{~km}$. This is quite different from the behaviour observed in Figure 12. In this case the cost-benefit ratio does have a substantial influence on the choice of threshold.

\section{Overall Conclusion}

Table 10 lists the threshold selection procedures studied in this paper, and summarises their performance. Ticks and crosses represent good and bad performance, respectively. Selection Performance is performance of the threshold selection procedure in estimating the threshold value. Reliability at Extremes is the reliability (inverse variance) of the criterion near the ends of the range of possible values of the threshold. Significance Test indicates whether there is a valid test of statistical significance for the presence of a threshold, directly linked to the criterion (validity 
means that the test does not violate the rule against cheating discussed in the subsection The Problem of "Peeking" in the section on SIGNIFICANCE TESTS. Min number of deposits gives a rule of thumb estimate of the minimum total number of deposits required for validity of the method. Robustness to failure of assumptions indicates the ability of the technique to give acceptable results when the simple threshold model does not hold.

The rule of thumb for the minimum total number of deposits assumes that the number of deposits inside the prospective region is at least twice the number outside the prospective region. Then the minimum total number of deposits is equal to three times the minimum number outside the prospective region, and the latter is determined by statistical rules for the various test procedures.

\begin{tabular}{|c|c|c|c|c|c|c|}
\hline METHOD & $\begin{array}{c}\text { Selection } \\
\text { Performance }\end{array}$ & $\begin{array}{l}\text { Reliability } \\
\text { at extremes }\end{array}$ & $\begin{array}{c}\text { Significance } \\
\text { test }\end{array}$ & $\begin{array}{l}\text { Min number } \\
\text { of deposits }\end{array}$ & $\begin{array}{c}\text { Robustness to failure } \\
\text { of assumptions }\end{array}$ & $\begin{array}{l}\text { Adaptability to } \\
\text { other models }\end{array}$ \\
\hline C & $x x$ & $x$ & & 30 & $x$ & $x$ \\
\hline$t$ & $\checkmark$ & $x$ & & 30 & $\checkmark$ & $x$ \\
\hline$\times 2$ & $\checkmark$ & $x$ & & 15 & $\checkmark$ & $\checkmark$ \\
\hline ARM & $\checkmark \checkmark$ & $x$ & A-R & 15 & $\checkmark$ & $\checkmark$ \\
\hline LL & $\checkmark \checkmark$ & $\checkmark$ & & 15 & $\checkmark \checkmark$ & $\checkmark \checkmark \checkmark$ \\
\hline$Y$ & $\checkmark \checkmark \checkmark$ & $\checkmark \checkmark \checkmark$ & K-S & 5 & $\checkmark \checkmark$ & $\checkmark$ \\
\hline
\end{tabular}

Table 10 Summary of methods for threshold selection reviewed in this paper, and their performance. Method abbreviations: C (WofE contrast), t (Studentised contrast), X2 ( $\chi^{2}$ statistic), ARM (Akman-Raftery criterion with constraints), LL (profile log-likelihood), and $\mathrm{Y}$ (Youden criterion). For column abbreviations, see text.

In our investigations, the method which selects the threshold value by maximising the WofE contrast $\widehat{C}$ had poor performance, both on real data and in simulation experiments. The Studentised contrast $t$ and the Akman-Raftery criterion performed reasonably well, although they have erratic behaviour when the candidate threshold is close to the smallest possible, or largest possible, threshold. The profile log-likelihood and Youden methods performed very well, and the Youden method had the best overall performance. The Youden, Akman-Raftery and $\chi^{2}$ methods are related (see the section MATHEMATICAL CONNECTIONS BETWEEN THE CRITERIA) in such a way that the Youden method will almost always have the best performance of the three. We recommend the Youden method because of its performance, its simplicity and because it can be used immediately to conduct a (Kolmogorov-Smirnov) test for existence of a threshold. Where the capture-efficiency curve indicates there may be more than one threshold, the profile log-likelihood is the most suitable.

WofE approaches have recently made the step from 2D to 3D modelling (Li et al. 2016; Payne et al. 2015; Wang et al. 2015; Xiao et al. 2015; Yang et al. 2017). The methods proposed in this paper do not depend on the spatial dimension, and could be applied to these 3D techniques.

\section{Caveats}

Our presentation has ignored numerous real-world complications, for lack of space.

The handling of missing data is important in real applications and is a major research field in statistical science (Alison 2002; Dempster et al. 1977; Harrell 2001; Hosmer and Lemeshow 2000; Little and Rubin 2002; Schafer 1997; Vach 1994; van Buuren 2012). It is not possible to do justice to this issue in the space available, but all the methods discussed here can be modified to handle missing data in the same way that is traditionally done for WofE.

Sample bias, due to preferential sampling or non-uniform survey effort, affects the data analysis because it affects the probability of detecting a deposit. The first two subsections above have discussed "misspecification", that is, the case where the real data do not obey the assumed model of a threshold relationship. One possible cause for this is a sampling bias effect. If the sampling bias is known (for example if the amount of survey effort in each region is known), then it can easily be accounted for in logistic regression (Baddeley 2018, p. 50, Baddeley et al. 2015, p. 302, 304). Further discussion is postponed to another paper.

For the two datasets considered in the paper, sample density bias is not a great concern. The synthetic dataset has been constructed artificially to follow an ideal threshold relationship. The Murchison dataset is regional in scale, and the source of the data is Geological Survey mapping at 1:250,000 scale, so that only major features are shown. Faults and shear zones were mapped using airborne magnetic survey data so the entire study area is likely to have been covered with equal detail.

Our study does not apply to unordered categorical predictors, such as rock types which are not organised in a sensible sequence. This is a different, and more challenging, problem because of the "peeking" fallacy (multiple 
testing effect) explained in the subsection The Problem of "Peeking" in the section on SIGNIFICANCE TESTS.

Other complications include possible dependence between grid cells due to spatial clustering of deposits, the different endowments of different deposits, and the physical size of the deposits.

\section{Acknowledgements}

We thank Kassel Hingee for his insightful contributions to the initial research work which led to this article. In initial phases of this research, Adrian Baddeley was funded by the Australian Research Council under a Discovery Outstanding Researcher Award, and hosted by Professor Eun-Jung Holden of the Centre for Exploration Targeting (CET) at the University of Western Australia. Aloke Phatak was partially supported by the Australian Government through the Australian Research Council's Industrial Transformation Training Centres scheme (project IC180100030). We warmly thank the reviewers for their comments, which have greatly helped us to improve the paper.

\section{Electronic Supplementary Material}

The online version of this article contains supplementary material which is available to authorised users.

\section{REFERENCES}

Agterberg, F. (1974). Automatic contouring of geological maps to detect target areas for mineral exploration. Journal of the International Association for Mathematical Geology, 6, 373-395.

Agterberg, F. (1992). Combining indicator patterns in weights of evidence modeling for resource evaluation. Nonrenewable Resources, 1, 39-50.

Agterberg, F. (2011). A modified weights-of-evidence method for regional mineral resource evaluation. Natural Resources Research, 20(2), 95-101.

Agterberg, F. (2014). Geomathematics: Theoretical Foundations, Applications, and Future Developments (No. 18). Cham: Springer.

Agterberg, F., \& Bonham-Carter, G. (1999). Logistic regression and weights of evidence modeling in mineral exploration. In K. Dagdalen (Ed.), Proceedings, 28th International Symposium on Computer Applications in the Mineral Industries - APCOM 99 (pp. 483-590). Golden, Colorado: Colorado School of Mines. (ISBN 0-918062-12-8)

Agterberg, F., \& Bonham-Carter, G. (2005). Measuring the performance of mineral-potential maps. Natural Resources Research, 14(1), 1-17.

Agterberg, F., Bonham-Carter, G., Cheng, Q., \& Wright, D. (1993). Weights of evidence modeling and weighted logistic regression for mineral potential mapping. In J. Davis \& U. Herzfeld (Eds.), Computers in Geology - 25 Years of Progress (pp. 13-32). New York: Oxford University Press.

Agterberg, F., \& Cheng, Q. (2002). Conditional independence test for weights-of-evidence modeling. Natural Resources Research, 11, 249-255.

Akman, V., \& Raftery, A. (1986). Asymptotic inference for a change-point Poisson process. Annals of Statistics, $14(4), 1583-1590$.

Alison, P. (2002). Missing Data. Thousand Oaks, CA: Sage.

Anonymous. (2011). "Significant". Retrieved from https://xkcd.com/882 (Web comic published on the XKCD website on 06 April 2011)

Baddeley, A. (2018). A statistical commentary on mineral prospectivity analysis. In B. D. Sagar, Q. Cheng, \& F. Agterberg (Eds.), Handbook of Mathematical Geosciences: Fifty Years of IAMG (pp. 25-65). International Association for Mathematical Geosciences.

Baddeley, A., Berman, M., Fisher, N., Hardegen, A., Milne, R., Schuhmacher, D., Shah, R., \& Turner, R. (2010). Spatial logistic regression and change-of-support for Poisson point processes. Electronic Journal of Statistics, 4, 1151-1201. doi: 10.1214/10-EJS581

Baddeley, A., Chang, Y., Song, Y., \& Turner, R. (2012). Nonparametric estimation of the dependence of a spatial point process on a spatial covariate. Statistics and its Interface, 5, 221-236.

Baddeley, A., Rubak, E., \& Turner, R. (2015). Spatial Point Patterns: Methodology and Applications with R. London: Chapman and Hall/CRC.

Baddeley, A., \& Turner, R. (2005). Spatstat: an R package for analyzing spatial point patterns. Journal of Statistical Software, 12(6), 1-42. (URL: www.jstatsoft.org, ISSN: 1548-7660) 
Ballantyne, C., \& Cornish, R. (1979). Use of the chi-square test for the analysis of orientation data. Journal of Sedimentary Research, 49(3), 773-776.

Barnard, G. (1959). Control charts and stochastic processes. Journal of the Royal Statistical Society, Series B, 21, 239-271.

Basseville, M., \& Nikiforov, I. (1993). Detection of Abrupt Changes: Theory and Applications. Englewood Cliffs, NJ: Prentice-Hall.

Berman, M. (1986). Testing for spatial association between a point process and another stochastic process. Applied Statistics, 35, 54-62.

Bhattacharya, G., \& Brockwell, P. (1976). The minimum of an additive process with applications to signal estimation and storage theory. Zeitschrift fuer Wahscheinlichkeitstheorie und verwandte Gebiete, 37, 5175 .

Bhattacharya, G., \& Johnson, R. (1968). Nonparametric tests for shift at unknown time point. Annals of Mathematical Statistics, 39, 1731-1743.

Bierlein, F., Murphy, F., Weinberg, R., \& Lees, T. (2006). Distribution of orogenic gold deposits in relation to fault zones and gravity gradients: targeting tools applied to the Eastern Goldfields, Yilgarn Craton, Western Australia. Mineralium Deposita, 41, 107-126.

Bierlein, F., Northover, H., Groves, D., Goldfarb, R., \& Marsh, E. (2008). Controls on mineralisation in the Sierra Foothills gold province, central California, USA: a GIS-based reconnaissance prospectivity analysis. Australian Journal of Earth Sciences, 55, 61-78.

Boleneus, D., Raines, G., Causey, J., Bookstrom, A., Frost, T., \& Hyndman, P. (2001). Assessment method for epithermal gold deposits in northeast Washington State using weights-of-evidence GIS modeling (Open-File Report Nos. 2001-501). US Geological Survey.

Bonham-Carter, G. (1995). Geographic Information Systems for Geoscientists: Modelling with GIS (No. 13). Kidlington, Oxford, UK: Pergamon Press/ Elsevier.

Bonham-Carter, G., \& Agterberg, F. (1990). Application of a microcomputer-based geographic information system to mineral-potential mapping. In J. T. Hanley \& D. F. Merriam (Eds.), Microcomputer Applications in Geology 2 (pp. 49-74). Amsterdam: Pergamon.

Bonham-Carter, G., Agterberg, F., \& Wright, D. (1990). Weights of evidence modelling: a new approach to mapping mineral potential. In F. Agterberg \& G. Bonham-Carter (Eds.), Statistical Applications in the Earth Sciences (pp. 171-183). Ottawa: Geological Survey of Canada. (Proceedings of the Colloquium on Statistical Applications in the Earth Sciences hosted by the Geological Survey of Canada in Ottawa on 14-18 November, 1988)

Breiman, L., Friedman, J., Stone, C., \& Olshen, R. (1984). Classification and Regression Trees. Chapman and Hall/CRC.

Brown, W. (2002). Artificial Neural Networks: A New Method for Mineral-Prospectivity Mapping (PhD thesis). University of Western Australia.

Brown, W., Gedeon, T., Baddeley, A., \& Groves, D. (2002). Bivariate J-function and other graphical statistical methods help select the best predictor variables as inputs for a neural network method of mineral prospectivity mapping. In U. Bayer, H. Burger, \& W. Skala (Eds.), IAMG 2002: 8th Annual Conference of the International Association for Mathematical Geology (Vol. 1, pp. 257-268).

Carlton, M., \& Devore, J. (2014). Probability with Applications in Engineering, Science, and Technology. New York: Springer. doi: 10.1007/978-1-4939-0395-5

Carranza, E. (2004). Weights of evidence modeling of mineral potential: a case study using small number of prospects, Abra, Philippines. Natural Resources Research, 13(3), 173-187.

Carranza, E. (2009). Data-driven modeling of mineral prospectivity. In M. Hale (Ed.), Handbook of Exploration and Environmental Geochemistry 11: Geochemical Anomaly and Mineral Prospectivity Mapping in GIS (pp. 249-310). Elsevier.

Cassard, D., Billa, M., Lambert, A., Picot, J., Husson, Y., \& Lassere, J. (2008). Gold predictivity mapping in French Guiana using an expert-guided data-driven approach based on a regional-scale GIS. Ore Geology Reviews, $34(3), 471-500$.

Cervi, F., Berti, M., Borgatti, L., Ronchetti, F., Manenti, F., \& Corsini, A. (2010). Comparing predictive capability of statistical and deterministic methods for landslide susceptibility mapping: a case study in the northern Apennines (Reggio Emilia Province, Italy). Landslides, 7(4), 433-444.

Chen, Y., \& Wu, W. (2019, January). Isolation forest as an alternative data-driven mineral prospectivity mapping method with a higher data-processing efficiency. Natural Resources Research, 28(1), 31-46.

Cheng, Q. (2004). Application of weights of evidence method for assessment of flowing wells in the greater Toronto area, Canada. Natural Resources Research, 13, 77-86. 
Cheng, Q. (2007). Mapping singularities with stream sediment geochemical data for prediction of undiscovered mineral deposits in Gejiu, Yunnan Province, China. Ore Geology Reviews, 32, 314-324.

Cheng, Q. (2008). Non-linear theory and power-law models for information integration and mineral resources quantitative assessments. Mathematical Geosciences, 40(5), 503-532.

Chernoff, H., \& Rubin, H. (1956). The estimation of the location of a discontinuity in density. In Proceedings, Third Berkeley Symposium on Mathematical Statistics and Probability (Vol. 1, pp. 19-37).

Chernoff, H., \& Zacks, S. (1964). Estimating the current mean of a normal distribution which is subjected to change in time. Annals of Mathematical Statistics, 35, 999-1018.

Chernoyarov, O., Kutoyants, Y., \& Top, A. (2018). On multiple change-point estimation for Poisson process. Communications in Statistics - Theory and Methods, 47(5), 1215-1233. doi: 10.1080/03610926.2017.1317810

Commenges, D., \& Seal, J. (1985). The analysis of neuronal discharge sequences: change-point estimation and comparison of variances. Statistics in Medicine, 4, 91-104.

Conover, W. (1999). Practical Nonparametric Statistics (Third ed.). New York: John Wiley and Sons.

Darkhovsky, B. (1976). A nonparametric method for the a posteriori detection of the "disorder" time of a sequence of independent random variables. Theory of Probability and its Applications, 21, 178-183.

Dempster, A. P., Laird, N. M., \& Rubin, D. B. (1977). Maximum likelihood from incomplete data via the E-M algorithm. Journal of the Royal Statistical Society B, 39, 1-22.

Deshayes, J. (1984). Ruptures de modèles pour les processus de Poisson. Annales Scientifiques Univ ClermontFerrand II, 78, 1-7.

Efron, B., \& Tibshirani, R. J. (1993). An Introduction to the Bootstrap (Vol. 57). London: Chapman and Hall.

Fabbri, A., \& Chung, C.-J. (2008). On blind tests and spatial prediction models. Natural Resources Research, $17(2), 107-118$.

Filzmosera, P., Garrett, R., \& Reimann, C. (2005). Multivariate outlier detection in exploration geochemistry. Computers and Geosciences, 31, 579-587.

Fisher, R. (1922). On the mathematical foundations of theoretical statistics. Philosophical Transactions of the Royal Society, Series A, 222(594-604), 309-368.

Ford, A., Miller, J., \& Mol, A. (2016). A comparative analysis of Weights of Evidence, evidential belief functions, and fuzzy logic for mineral potential mapping using incomplete data at the scale of investigation. Natural Resources Research, 25, 19-33.

Foxall, R., \& Baddeley, A. (2002). Nonparametric measures of association between a spatial point process and a random set, with geological applications. Applied Statistics, 51(2), 165-182.

Galun, S., \& Trifonov, A. (1982). Detection and estimation of the time when the Poisson flow intensity changes. Automation and Remote Control, 43(6), 782-790.

Gardner, L. (1969). On detecting changes in the mean of normal variables. Annals of Mathematical Statistics, 40, 116-126.

Garrett, R. (1989). The chi-square plot: a tool for multivariate outlier recognition. Journal of Geochemical Exploration, 32(1), 319-341.

Geological Survey of Western Australia. (1994). (https://dmp.wa.gov.au/Mines-and-mineral-deposits-1502.aspx)

MINEDEX database.

Ghannadpour, S., \& Hezarkhani, A. (2016). Exploration geochemistry data-application for anomaly separation based on discriminant function analysis in the Parkam porphyry system. Geosciences Journal, 20(6), 837850.

Goldfarb, R., \& Groves, D. (2015). Orogenic gold: Common or evolving fluid and metal sources through time. Lithos, 233, 2-26.

Goodacre, A., Bonham-Carter, G., Agterberg, F., \& Wright, D. (1993). A statistical analysis of the spatial association of seismicity with drainage patterns and magnetic anomalies in western Quebec. Tectonophysics, 217, 285-305.

Gorney, R., Ferris, D., Ward, A., \& Williams, L. (2011). Assessing channel-forming characteristics of an impacted headwater stream in Ohio, USA. Ecological Engineering, 37(3), 418-430.

Groves, D., Goldfarb, R., Knox-Robinson, C., Ojala, J., Gardoll, S., Yun, G., \& Holyland, P. (2000). Late-kinematic timing of orogenic gold deposits and significance for computer-based exploration techniques with emphasis on the Yilgarn Block, Western Australia. Ore Geology Reviews, 17, 1-38.

Groves, D., \& Santosh, M. (2016). The giant Jiaodong gold province: The key to a unified model for orogenic gold deposits? Geoscience Frontiers, 7, 409-417.

Hájek, J., \& Rényi, A. (1955). Generalization of an inequality of Kolmogorov. Acta Math. Acad. Sci. Hungar., 6, 281-283.

Harrell, F. (2001). Regression Modeling Strategies. New York: Springer. 
Harris, D., \& Pan, G. (1999). Mineral favourability mapping: a comparison of artificial neural networks, logistic regression, and discriminant analysis. Natural Resources Research, 8, 93-109.

Harris, D., Zurcher, L., Stanley, M., Marlow, J., \& Pan, G. (2003). Comparative analysis of favorability mappings by weights of evidence, probabilistic neural networks, discriminant analysis, and logistic regression. Natural Resources Research, 12(4), 241-255.

Harris, J., Grunsky, E., Behnia, P., \& Corrigan, D. (2015). Data- and knowledge-driven mineral prospectivity maps for Canada's North. Ore Geology Reviews, 71, 788-803.

Hinkley, D. (1970). Inference about the change-point in a sequence of random variables. Biometrika, 57, $1-17$.

Hinkley, D. (1971). Inference about the change-point from the cumulative sum test. Biometrika, 58, 509-523.

Hochberg, Y., \& Tamhane, A. (1987). Multiple Comparison Procedures. New York: John Wiley and Sons.

Hogg, R., \& Craig, A. (1970). Introduction to Mathematical Statistics (Third ed.). Macmillan.

Hosmer, D., \& Lemeshow, S. (2000). Applied Logistic Regression (Second ed.). John Wiley and Sons.

Hsu, J. (1996). Multiple Comparisons: Theory and Methods. Chapman and Hall.

Kalbfleisch, J. (1985). Probability and Statistical Inference. Volume 2: Statistical Inference (Second ed.). New York: Springer.

Kander, Z., \& Zacks, S. (1966). Test procedures for possible changes in parameters of statistical distributions occuring at unknown time points. Annals of Mathematical Statistics, 37, 1196-1210.

Kendall, M. G., \& Stuart, A. (1973). The Advanced Theory of Statistics (Third ed., Vol. 2). London: Charles Griffin and Company Ltd.

Knox-Robinson, C., \& Groves, D. (1997). Gold prospectivity mapping using a geographic information system (GIS), with examples from the Yilgarn Block of Western Australia. Chronique de la Recherche Minière, 529, 127-138.

Krzanowski, W., \& Hand, D. (2009). ROC Curves for Continuous Data. London/Boca Raton: Chapman and Hall/CRC Press.

Kutoyants, Y. (1998). Statistical Inference for Spatial Poisson Processes (No. 134). New York: Springer.

Lehmann, E. L. (1999). Elements of Large-Sample Theory. New York: Springer-Verlag.

Leonard, T. (1978). Density estimation, stochastic processes and prior information (with discussion). Journal of the Royal Statistical Society, Series B, 40, 113-146.

Li, N., Bagas, L., Li, X., Xiao, K., Li, Y., Ying, L., \& Song, X. (2016). An improved buffer analysis technique for model-based 3D mineral potential mapping and its application. Ore Geology Reviews, 76, 94-107.

Lindsey, J. (1996). Parametric Statistical Inference. Oxford: Clarendon Press.

Little, R., \& Rubin, D. (2002). Statistical Analysis with Missing Data (Second ed.). John Wiley and Sons.

Liu, J., \& Cheng, Q. (2019). A modified Weights-of-Evidence method for mineral potential prediction based on structural equation modeling. Natural Resources Research, 28, 1037-1053.

Liu, Y., Cheng, Q., Xia, Q., \& Wang, X. (2014). Mineral potential mapping for tungsten polymetallic deposits in the Nanling metallogenic belt, South China. Journal of Earth Science, 25, 689-700.

Loader, C. (1992). A log-linear model for a Poisson process changepoint. Annals of Statistics, 20, 1391-1411.

Murphy, S., \& van der Vaart, A. (2000). On profile likelihood. Journal of the American Statistical Association, 95(450), 449-465.

Nam, B.-H., \& D'Agostino, R. (2002). Discrimination index, the area under the ROC curve. In C. Huber-Carol, N. Balakrishnan, M. Nikulin, \& M. Mesbah (Eds.), Goodness-of-Fit Tests and Model Validity (pp. 267-279). Basel: Birkhäuser.

Neuhäuser, B., \& Terhorst, B. (2007). Landslide susceptibility assessment using "weights-of-evidence" applied to study area at the Jurassic escarpment (SW-Germany). Geomorphology, 86(1-2), 12-24.

Page, E. (1954). Continuous inspection schemes. Biometrika, 41, 100-115.

Page, E. (1957). On problems in which a change in a parameter occurs at an unknown point. Biometrika, 44, $248-252$

Payne, C., Cunningham, F., Peters, K., Nielsen, S., Puccioni, E., Wildman, C., \& Partington, G. (2015). From 2D to 3D: Prospectivity modelling in the Taupo Volcanic Zone, New Zealand. Ore Geology Reviews, 71, $558-577$.

Pearson, K. (1900). On the criterion that a given system of deviations from the probable in the case of a correlated system of variables is such that it can be reasonably supposed to have arisen from random sampling. Philosophical Magazine, 50(302), 157-175. (Series 5)

Pflug, G. (1983). The limiting log-likelihood process for discontinuous density families. Zeitschrift fuer Wahscheinlichkeitstheorie und verwandte Gebiete, 64, 15-35.

Polykretis, C., \& Chalkias, C. (2018). Comparison and evaluation of landslide susceptibility maps obtained from weights of evidence, logistic regression, and artificial neural network models. Natural Resources Research, 
93, $249-274$.

Pons, O. (2018). Estimations and Tests in Change-Point Models. Singapore: World Scientific.

Porwal, A., Gonzalez-Alvarez, I., Markwitz, V., McCuaig, T., \& Mamuse, A. (2010). Weights-of-evidence and logistic regression modeling of magmatic nickel sulfide propectivity in the Yilgarn Craton, Western Australia. Ore Geology Reviews, 38(3), 184-196.

Pratt, J. (1959). On a general concept of "In Probability". Annals of Mathematical Statistics, 30, 549-558.

R Development Core Team. (2018). R: A language and environment for statistical computing [Computer software manual]. Vienna, Austria. Retrieved from http://www.R-project.org/ (ISBN 3-900051-07-0)

Raftery, A., \& Akman, V. (1986). Bayesian analysis of a Poisson process with a change-point. Biometrika, 73(1), 85-89.

Read, T., \& Cressie, N. (1988). Goodness-of-Fit Statistics for Multivariate Data. New York: Springer-Verlag.

Rice, J. (2006). Mathematical Statistics and Data Analysis (Third ed.). New York: Duxbury.

Robert, F., Poulson, K., Cassidy, K., \& Hodgson, C. (2005). Gold metallogeny of the Superior and Yilgarn Cratons. In J. Hedenquist, J. Thompson, R. Goldfarb, \& J. Richards (Eds.), Economic Geology One Hundredth Anniversary Volume (pp. 1001-1033). Littleton, Colorado, USA: Society of Economic Geologists. (ISBN 978-1-887483-01-8)

Romero-Calcerrada, R., Barrio-Parra, F., Millington, J., \& Novillo, C. (2010). Spatial modeling of socioeconomic data to understand patterns of human-caused wildfire ignition risk in the SW of Madrid (central Spain). Ecological Modelling, 221(1), 34-45.

Romero-Calcerrada, R., \& Luque, S. (2006). Habitat quality assessment using Weights-of-Evidence based GIS modelling: The case of Picoides tridactylus as species indicator of the biodiversity value of the Finnish forest. Ecological Modelling, 196(1-2), 62-76.

Rubin, H. (1961). The estimation of discontinuities in multivariate densities, and related problems in stochastic process. In Proceedings, Fourth Berkeley Symposium on Mathematical Statistics and Probability (Vol. 1, pp. 563-574). University of California Press.

Ruopp, M., Perkins, N., Whitcomb, B., \& Schisterman, E. (2008, June). Youden index and optimal cut-point estimated from observations affected by a lower limit of detection. Biometrical journal, 50(3), 419-430.

Sager, T. (1982). Nonparametric maximum likelihood estimation of spatial patterns. Annals of Statistics, 10, $1125-1136$.

Schaeben, H. (2014). Targeting: logistic regression, special cases and extensions. ISPRS International Journal of Geo-Information, 3, 1387-1411.

Schaeben, H., \& Semmler, G. (2016). The quest for conditional independence in prospectivity modeling: weightsof-evidence, boost weights-of-evidence, and logistic regression. Frontiers of Earth Science, 10(3), 389-408.

Schafer, J. (1997). Analysis of Incomplete Multivariate Data. London: Chapman and Hall.

Sen, A., \& Srivastava, M. (1975). On tests for detecting change in mean. Annals of Statistics, 3, 98-108.

Severini, T. (2000). Likelihood Methods in Statistics. Oxford: Oxford University Press.

Shaffer, J. P. (1995). Multiple hypothesis testing. Annual Review of Psychology, 46, 561 - 584.

Shewhart, W. (1983). Economic Control of Quality of Manufactured Product. Princeton, NJ: Van Nostrand Reinhold.

Silverman, B. (1986). Density Estimation for Statistics and Data Analysis. London: Chapman and Hall.

Smith, A. (1975). A Bayesian approach to inference about a change-point in a sequence of random variables. Biometrika, 62, 407-416.

Solomon, M., \& Groves, D. (1994). The Geology and Origin of Australia's Mineral Deposits. New York: Oxford University Press.

Stephens, M. (1986). Tests based on EDF statistics. In R. D'Agostino \& M. Stephens (Eds.), Goodness-of-Fit Techniques (Vol. 68, pp. 97-193). New York: Marcel Dekker.

Vach, W. (1994). Logistic Regression with Missing Values in the Covariates. Berlin: Springer-Verlag.

van Buuren, S. (2012). Flexible Imputation of Missing Data. Boca Raton: Chapman and Hall.

Wand, M., \& Jones, M. (1995). Kernel Smoothing. Chapman and Hall.

Wang, G., Du, W., \& Carranza, E. (2016). Remote sensing and GIS prospectivity mapping for magmatichydrothermal base- and precious-metal deposits in the Honghai district, China. Journal of African Earth Sciences, 128, 97-115.

Wang, G., Li, R., Carranza, E., Zhang, S., Yan, C., Zhu, Y., Qu, J., Hong, D., Song, Y., Han, J., Ma, Z., Zhang, H., \& Yang, F. (2015). 3D geological modeling for prediction of subsurface Mo targets in the Luanchuan district, China. Ore Geology Reviews, 71, 592-610.

Wasserman, L. (2004). All of Statistics: a Concise Course in Statistical Inference. New York: Springer.

Watkins, K., \& Hickman, A. (1990). Geological evolution and mineralization of the Murchison Province, Western 
Australia (Bulletin No. 137). Geological Survey of Western Australia. (Published by Department of Mines, Western Australia, 1990. Available online from Department of Industry and Resources, State Government of Western Australia, www.doir.wa.gov.au)

West, W., \& Ogden, T. (1997). Continuous-time estimation of a changepoint in a Poisson process. Journal of Statistical Computation and Simulation, 56(4), 293-302.

Wilk, M., \& Gnanadesikan, R. (1968). Probability plotting methods for the analysis of data. Biometrika, 55, $1-17$.

Witt, W., Ford, A., Hanrahan, B., \& Mamuse, A. (2013). Regional-scale targeting for gold in the Yilgarn Craton: Part 1 of the Yilgarn Gold Exploration Targeting Atlas (Report No. 125). Perth, Western Australia: Geological Survey of Western Australia.

Xiao, K., Li, N., Porwal, A., Holden, E., Bagas, L., \& Lu, Y. (2015). GIS-based 3D prospectivity mapping: A case study of Jiama copper-polymetallic deposit in Tibet, China. Ore Geology Reviews, 71, 611-632.

Yang, F., Wang, G., Santosh, M., Li, R., Tang, L., Cao, H., Guo, N., \& Liu, C. (2017). Delineation of potential exploration targets based on 3D geological modeling: A case study from the Laoangou $\mathrm{Pb}-\mathrm{Zn}-\mathrm{Ag}$ polymetallic ore deposit, China. Ore Geology Reviews, 89, 228-252.

Yeomans, C. (2018). Enhancing the Geological Understanding of Southwest England using Machine Learning Algorithms (Unpublished doctoral dissertation). Camborne School of Mines.

Youden, W. (1950). Index for rating diagnostic tests. Cancer, 3, 32-35.

Zacks, S. (1983). Survey of classical and Bayesian approaches to the change-point problem: Fixed sample and sequential procedures of testing and estimation. In M. Rizvi, J. Rustagi, \& D. Siegmund (Eds.), Recent Advances in Statistics: Papers in Honour of Herman Chernoff on His Sixtieth Birthday (pp. 245-269). New York/London: Academic Press.

Zhang, N., \& Zhou, K. (2015). Mineral prospectivity mapping with weights of evidence and fuzzy logic methods. Journal of Intelligent and Fuzzy Systems, 29(6), 2639-2651. 


\section{APPENDICES}

\section{Capture-Efficiency Curve as a CDF}

In the section on THRESHOLD SELECTION USING THE CAPTURE-EFFICIENCY CURVE, subsection Principle, we mentioned that the capture-efficiency curve can be regarded as a cumulative distribution function (cdf) in its own right. Here we clarify that comment.

A very subtle interpretation of the capture-efficiency curve used by statisticians is the "transformation to uniformity" or "probability integral transformation" (Kendall and Stuart 1973, p. 459 ff.; Hogg and Craig 1970, pp. 349-350). Suppose that the original predictor $Z$ is replaced by a new predictor $V$, defined at each spatial location $u$ by $V(u)=G(Z(u))=a(Z(u)) / a$, which is the area fraction of spatial locations where the predictor value does not exceed the value $z$. In words, at a given spatial location $u$, the value of $V(u)$ is the fraction of area of the survey region where the original predictor $Z$ does not exceed the value $Z(u)$ which it takes at $u$. If $Z$ is distance-to-nearest-fault, then $V(u)$ is the area fraction occupied by the buffer at distance equal to the distance from $u$ to the faults, that is, the buffer whose boundary passes through the point $u$. Then the capture-efficiency curve is the cumulative distribution function of the transformed predictor $V$ at the deposit points, while the diagonal line is the cumulative distribution function of $V$ over all spatial locations in the survey region.

\section{Likelihood Function for Threshold Model}

This appendix provides elementary explanations for the appearance of the binomial probability distribution discussed in the section on SIGNIFICANCE TESTS, subsection Significance Test for a Binary Predictor, and for the form of the likelihood function based on this distribution, and for the form of the likelihood discussed in the section on THRESHOLD SELECTION USING CHANGE-POINT ANALYSIS, sub-subsection Profile Likelihood for the Threshold Model, which results from the assumption that the grid cells are very small in area relative to the survey region.

To simplify discussion, we assume that the geometry of the survey region $S$ and of the prospective map feature $B$ are fixed and known in advance, while the mineral deposit locations are discovered during the survey. In the notation of the section on BINARY PREDICTORS, the survey region has area $a$ and the feature $B$ has area $a_{B}$.

The survey process begins by dividing the survey region into $N$ grid cells of equal area, then determining whether each grid cell contains or does not contain a deposit. For our purposes the results of the survey are the count $n_{B}$ of grid cells inside $B$ which contain a deposit, and the count $n_{\bar{B}}$ of grid cells outside $B$ which contain a deposit. The total number of grid cells containing deposits is $n=n_{B}+n_{\bar{B}}$.

\section{Uniform Prospectivity Model Using Binomial Probabilities}

We first consider the simple model in which prospectivity is uniform over the entire survey region. Each grid cell has the same probability $p$ of containing a deposit. The outcomes in different grid cells are assumed to be statistically independent. There are $N$ grid cells altogether. Therefore the number $n$ of grid cells containing a deposit follows a binomial distribution on $N$ trials with success probability $p$; the probability that exactly $n$ grid cells contain deposits is

$$
\left(\begin{array}{c}
N \\
n
\end{array}\right) p^{n}(1-p)^{N-n}
$$

The expected total number of grid cells which contain deposits is $N p$.

The same principle applies to the grid cells inside the feature $B$; the probability that exactly $n_{B}$ grid cells inside $B$ contain deposits is

$$
\left(\begin{array}{c}
N_{B} \\
n_{B}
\end{array}\right) p^{n_{B}}(1-p)^{N_{B}-n_{B}},
$$

where $N_{B}=\left(a_{B} / a\right) N$ is the number of grid cells that constitute the feature $B$. The expected number of grid cells inside $B$ that contain deposits is $p N_{B}=p\left(a_{B} / a\right) N$.

Again this applies to the grid cells outside $B$; the probability of obtaining $n_{\bar{B}}$ grid cells outside $B$ which contain deposits is

$$
\left(\begin{array}{c}
N_{\bar{B}} \\
n_{\bar{B}}
\end{array}\right) p^{n_{\bar{B}}}(1-p)^{N_{\bar{B}}-n_{\bar{B}}}
$$


In the simple threshold model we assume that prospectivity is higher inside the feature $B$. Each grid cell inside $B$ has probability $p_{B}$ of containing a deposit, while each grid cell outside $B$ has a different probability $p_{\bar{B}}$ of containing a deposit, where $p_{\bar{B}}<p_{B}$. We simply replace $p$ by $p_{B}$ in equation (27) to find that the probability of obtaining exactly $n_{B}$ cells with deposits inside $B$ is

$$
\left(\begin{array}{c}
N_{B} \\
n_{B}
\end{array}\right) p_{B}^{n_{B}}\left(1-p_{B}\right)^{N_{B}-n_{B}} .
$$

Replacing $p$ by $p_{\bar{B}}$ in equation (28), the probability of obtaining $n_{\bar{B}}$ grid cells outside $B$ which contain deposits is

$$
\left(\begin{array}{c}
N_{\bar{B}} \\
n_{\bar{B}}
\end{array}\right) p_{\bar{B}}^{n_{\bar{B}}}\left(1-p_{\bar{B}}\right)^{N_{\bar{B}}-n_{\bar{B}}} .
$$

Combining (31) and (32), the probability of obtaining $n_{B}$ cells containing deposits inside $B$ and $n_{\bar{B}}$ cells containing deposits outside $B$ is

$$
\left(\begin{array}{c}
N_{B} \\
n_{B}
\end{array}\right) p_{B}^{n_{B}}\left(1-p_{B}\right)^{N_{B}-n_{B}}\left(\begin{array}{c}
N_{\bar{B}} \\
n_{\bar{B}}
\end{array}\right) p_{\bar{B}}^{n_{\bar{B}}}\left(1-p_{\bar{B}}\right)^{N_{\bar{B}}-n_{\bar{B}}}
$$

The likelihood function for the simple threshold model is (again omitting constant factors)

$$
L\left(p_{B}, p_{\bar{B}}\right)=p_{B}^{n_{B}}\left(1-p_{B}\right)^{N_{B}-n_{B}} p_{\bar{B}}^{n_{\bar{B}}}\left(1-p_{\bar{B}}\right)^{N_{\bar{B}}-n_{\bar{B}}} .
$$

The likelihood now has two arguments $p_{B}$ and $p_{\bar{B}}$ representing the probabilities of a deposit for grid cells inside and outside $B$, respectively. Notice that if the two probabilities were equal, $p_{B}=p_{\bar{B}}=p$, then (33) would collapse to $(30)$.

\section{Rescaling in Terms of Density}

Since the grid cells are artificial (and in particular their size is an arbitrary choice) it is useful to rescale the equations so that they depend as little as possible on the grid geometry. This can be done by using the average density of deposits, $\mu$, defined as the expected number per unit area.

In the uniform prospectivity model, the expected total number of grid cells that contain deposits is $N p$. The average density is therefore $\mu=N p / a$. Noting that the area of one grid cell is $\epsilon=a / N$, we see that the average density is equal to $\mu=p / \epsilon$. Equivalently, $p=\mu \epsilon$, that is, the probability of a deposit in any given cell is equal to the average density of deposits times the area of the grid cell. Replacing $p$ by $\mu \epsilon$ in equation (30), and removing constant factors, we get the likelihood

$$
L(\mu)=\mu^{n}(1-\mu \epsilon)^{N-n} .
$$

In the simple threshold model, we have two different densities inside and outside the feature $B$. Inside $B$, the expected number of grid cells containing deposits is $N_{B} p_{B}$ and the area is $a_{B}=N_{B} \epsilon$ so the density is $\mu_{B}=N_{B} p_{B} / a_{B}=p_{B} / \epsilon$. Outside $B$, the density is $\mu_{\bar{B}}=p_{\bar{B}} N_{\bar{B}} / a_{\bar{B}}=p_{\bar{B}} / \epsilon$. Substituting into (33) we get the likelihood

$$
L\left(\mu_{B}, \mu_{\bar{B}}\right)=\mu_{B}^{n_{B}}\left(1-\epsilon \mu_{B}\right)^{N_{B}-n_{B}} \mu_{\bar{B}}^{n_{\bar{B}}}\left(1-\epsilon \mu_{\bar{B}}\right)^{N_{\bar{B}}-n_{\bar{B}}} .
$$


Small Grid Cells

Finally, we suppose that the grid cells are very small. Then $N$ is very large, $\epsilon=a / N$ is very small. For the uniform prospectivity model, in the likelihood $(30)$ the term $(1-\mu \epsilon)^{N-n}$ converges to the exponential $\exp (-\mu N \epsilon)=$ $\exp (-\mu a)$ as $N$ becomes large, giving the likelihood

$$
L(\mu)=\mu^{n} \exp (-\mu a) .
$$

For the simple threshold model, the likelihood (33) similarly converges to

$$
L\left(\mu_{B}, \mu_{\bar{B}}\right)=\mu_{B}^{n_{B}} \mu_{\bar{B}}^{n_{\bar{B}}} \exp \left(-\mu_{B} a_{B}-\mu_{\bar{B}} a_{\bar{B}}\right) .
$$

Taking logarithms, the log-likelihood for the null model of uniform prospectivity is

$$
\ln L(\mu)=n \ln \mu-\mu a,
$$

and for the simple threshold model

$$
\ln L\left(\mu_{B}, \mu_{\bar{B}}\right)=n_{B} \ln \mu_{B}+n_{\bar{B}} \ln \mu_{\bar{B}}-\mu_{B} a_{B}-\mu_{\bar{B}} a_{\bar{B}} .
$$

If the feature $B$ is the region determined by thresholding a spatial predictor function $Z(u)$ to have values less than or equal to a threshold $z$, then $a_{B}=a(z)$ is the area of this region, $n_{B}=n(z)$ is the number of deposits with predictor values less than or equal to $z$, and we have $a_{\bar{B}}=a-a_{B}$ and $n_{\bar{B}}=n-n_{B}=n-n(z)$, so that equation (37) is translated into equation (10) of the paper.

\section{Connections Between the Threshold Selection Criteria}

In this appendix, we provide proofs of claims made in the sections SIGNIFICANCE TESTS and MATHEMATICAL CONNECTIONS BETWEEN THE CRITERIA regarding relationships between various of the threshold selection criteria.

\section{Forms of the $\chi^{2}$ Statistic}

First we prove the claim in the section on SIGNIFICANCE TESTS that the general form of the $\chi^{2}$ statistic (18) reduces to the special form (19) in this case. For any feature $B$, define $e_{B}=(n / a) a_{B}=\left(a_{B} / a\right) n$ and $e_{\bar{B}}=$ $(n / a) a_{\bar{B}}=\left(a_{\bar{B}} / a\right) n$, the expected counts in $B$ and $\bar{B}$, respectively, when the deposits are randomly distributed with constant density. Then

$$
n_{\bar{B}}-e_{\bar{B}}=\left(n-n_{B}\right)-\left(n-e_{B}\right)=-\left(n_{B}-e_{B}\right)
$$

so that

$$
\begin{aligned}
X^{2} & =\frac{\left(n_{B}-e_{B}\right)^{2}}{e_{B}}+\frac{\left(n_{\bar{B}}-e_{\bar{B}}\right)^{2}}{e_{\bar{B}}}=\frac{\left(n_{B}-e_{B}\right)^{2}}{e_{B}}+\frac{\left(n_{B}-e_{B}\right)^{2}}{e_{\bar{B}}}=\left(n_{B}-e_{B}\right)^{2}\left(\frac{1}{e_{B}}+\frac{1}{e_{\bar{B}}}\right) \\
& =\left(n_{B}-e_{B}\right)^{2} \frac{e_{\bar{B}}+e_{B}}{e_{B} e_{\bar{B}}}=\frac{n}{e_{B} e_{\bar{B}}}\left(n_{B}-e_{B}\right)^{2},
\end{aligned}
$$

which is equivalent to (19).

\section{Connection Between Akman-Raftery and $\chi^{2}$ Criteria}

Next we prove the connection between $\operatorname{AR}(z)$ and $X(z)$ stated in equation (14), in the section on MATHEMATICAL CONNECTIONS BETWEEN THE CRITERIA, and again in the section on SIGNIFICANCE TESTS, subsection Akman-Raftery (Constrained $\chi^{2}$ ) Test.

For candidate threshold value $z$, let $n(z)$ be the number of deposits below the threshold, $a(z)$ the area of study region below the threshold, $n$ the total number of deposits, $a$ the total area of study region. Define $s(z)=a(z) / a$, the area fraction. 
Then from the definition (13) of the Akman-Raftery criterion,

$$
\begin{aligned}
\operatorname{AR}(z) & =\sqrt{s(z)(1-s(z))}\left(\frac{n(z)}{s(z)}-\frac{n-n(z)}{1-s(z)}\right) \\
& =\sqrt{s(z)(1-s(z))} \frac{n(z)(1-s(z))-s(z)(n-n(z))}{s(z)(1-s(z))} \\
& =\sqrt{s(z)(1-s(z))} \frac{n(z)-n s(z)}{s(z)(1-s(z))} \\
& =n \frac{\frac{n(z)}{n}-s(z)}{\sqrt{s(z)(1-s(z))}} \\
& =\sqrt{n} X(z) .
\end{aligned}
$$

This proves (14).

\section{Relation Between $\chi^{2}$ Statistic and Youden Criterion}

Here, we prove the claim, made in the section MATHEMATICAL CONNECTIONS BETWEEN THE CRITERIA, that the $\chi^{2}$ statistic is the standardised version of the Youden criterion.

Under the null hypothesis that the density of deposits is uniform, if we treat the total number of deposits as fixed, then for any threshold $z$ the count $n(z)$ follows a binomial distribution with $n$ trials and success probability $p=a(z) / a=s(z)$. This distribution has variance $n p(1-p)=n s(z)(1-s(z))$. Accordingly $Y(z)=(n(z) / n)-s(z)$ has variance $s(z)(1-s(z)) / n$ so that the standard error of $Y(z)$, under the null hypothesis of a uniform density of deposits, is $\operatorname{se}_{0}(Y(z))=\sqrt{s(z)(1-s(z)) / n}$. Finally, inspecting (7) yields (15).

\section{Derivation of Optimal Threshold for Gradual Decline in Prospectivity}

This appendix provides the proof of the claim in the subsection Gradual Decline in Prospectivity of the DISCUSSION that, if the prospectivity (i.e. the density of deposit points) is a decreasing function of the predictor value, then the optimal threshold for the Youden criterion is the threshold at which that function equals the average density of deposits over the survey region.

We define the prospectivity as the spatially-varying density (intensity) of deposit points considered as a function $\lambda(u)$ of spatial location $u$. In any given grid cell, the expected number of deposits is equal to $\lambda(u) \epsilon$ where $u$ is the location of the cell centre and $\epsilon>0$ is the cell area. See Baddeley (2018) for an explanation.

Assume first that the density of deposits depends only on the predictor $Z$. That is, we assume that $\lambda(u)$ depends on $Z(u)$ through the relation $\lambda(u)=\rho(Z(u))$, where $\rho$ is a nonnegative function. Several examples of the relationship between predictor and prospectivity are shown in the top row of Figure 23; these are graphs of $\rho(z)$ against $z$. Assume further, as we did in subsection Gradual Decline in Prospectivity, that $\rho(z)$ is a decreasing function of $z$, such as those in the left and right columns of Figure 23. Then, $G(z)$ is the spatial cumulative distribution function of the predictor over the study region $S$, that is,

$$
G(z)=\frac{1}{a} \int_{S} \mathbf{1}\{Z(u) \leq z\} \mathrm{d} u,
$$

where $\mathbf{1}\{Z(u) \leq z\}$ is the indicator function, equal to 1 if $Z(u) \leq z$ and equal to 0 otherwise. The expected total number of deposits $\mathbb{E}[n]$ is equal to the integral of the intensity function,

$$
\mathbb{E}[n]=\int_{S} \lambda(u) \mathrm{d} u=\int_{S} \rho(Z(u)) \mathrm{d} u
$$

This integral over the spatial domain $S$ can be transformed into a one-dimensional integral

$$
\mathbb{E}[n]=a \int_{-\infty}^{\infty} \rho(z) \mathrm{d} G(z) .
$$

The cumulative distribution function of the values of $Z$ at the deposit points, averaged over all random outcomes, is

$$
\widetilde{F}(z)=\frac{a \int_{-\infty}^{z} \rho(v) \mathrm{d} G(v)}{a \int_{-\infty}^{\infty} \rho(v) \mathrm{d} G(v)}=\frac{1}{\mu} \int_{-\infty}^{z} \rho(v) \mathrm{d} G(v),
$$


where $\mu=\mathbb{E}[n] / a$ is the average density of deposits over the whole domain, and $v$ is the dummy variable of integration.

The expected capture-efficiency curve is the graph of $\widetilde{F}(z)$ against $G(z)$ for all $z$; equivalently it is the graph of the function $s \mapsto \widetilde{F}\left(G^{-1}(s)\right)$ where $G^{-1}$ is the inverse function of $G$. Assuming differentiability, the slope of the expected capture-efficiency curve is

$$
\frac{\mathrm{d}}{\mathrm{d} s} \widetilde{F}\left(G^{-1}(s)\right)=\frac{\widetilde{F}^{\prime}\left(G^{-1}(s)\right)}{G^{\prime}\left(G^{-1}(s)\right)} .
$$

But from (38) we have $\widetilde{F}^{\prime}(z)=\rho(z) G^{\prime}(z) / \mu$, so that the slope of the expected capture-efficiency curve at a given area fraction $s$ is equal to $\rho(z) / \mu$, where $z=G^{-1}(s)$.

The Youden method selects the point on the capture-efficiency curve with slope equal to 1. Ignoring sampling variability, that is, if we replace the observed capture-efficiency curve by the expected capture-efficiency curve, the Youden method selects the point with slope $\rho(z) / \mu=1$, i.e., it selects the threshold value $z$ for which $\rho(z)=\mu$.

\section{Non-negligible Grid Cell Size}

Equations in the paper assume, for simplicity and clarity, that the area of a grid cell is negligible. To be precise, if $\epsilon$ denotes the area of one grid cell, then we assume that $n \epsilon$, the total area of all cells containing deposits, can be treated as zero. This appendix lists the modifications to these equations that are necessary when $n \epsilon$ is not negligible.

In equation (1), the revised formulae are

$$
\begin{aligned}
W_{+} & =\ln \frac{n_{B}}{a_{B}-\epsilon n_{B}}-\ln \frac{n}{a} \\
W_{-} & =\ln \frac{n_{\bar{B}}}{a_{\bar{B}}-\epsilon n_{\bar{B}}}-\ln \frac{n}{a} \\
\widehat{C} & =\ln \frac{n_{B}}{a_{B}-\epsilon n_{B}}-\ln \frac{n_{\bar{B}}}{a_{\bar{B}}-\epsilon n_{\bar{B}}} .
\end{aligned}
$$

Equation (2) becomes

$$
\widehat{C}(z)=\ln \left(\frac{n(z)}{a(z)-\epsilon n(z)} / \frac{n-n(z)}{a-a(z)-\epsilon(n-n(z))}\right) .
$$

Equation (3) becomes

$$
\operatorname{se}(\widehat{C})=\sqrt{\frac{1}{n_{B}}+\frac{1}{n_{\bar{B}}}+\frac{1}{a_{B} / \epsilon-n_{B}}+\frac{1}{a_{\bar{B}} / \epsilon-n_{\bar{B}}}}
$$

and equation (5) becomes

$$
\operatorname{se}(\widehat{C}(z))=\sqrt{\frac{1}{n(z)}+\frac{1}{n-n(z)}+\frac{1}{a(z) / \epsilon-n(z)}+\frac{1}{(a-a(z)) / \epsilon-(n-n(z))}} .
$$

Equation (8) for the Youden criterion becomes

$$
Y(z)=\frac{n(z)}{n}-\frac{a(z)-\epsilon n(z)}{a-\epsilon n} .
$$

There are no changes in other equations, except that equation (12) does not hold. In the right panel of Figure 10, the ROC curve should be used instead of the capture-efficiency curve. 


\title{
Online Resource 1
}

\author{
for the article \\ Optimal thresholding of predictors in mineral prospectivity analysis
}

Baddeley et al. (2020)

This is a demonstration of our computer code for optimal thresholding of greyscale predictors that was used in the original article. The code and original data are provided in auxiliary files.

This document was produced from the original script file OnlineResourcel. Rnw by applying the R command Sweave.

The original script file contains a mixture of $\mathrm{R}$ code and $\mathrm{LT}_{\mathrm{E}} \mathrm{X}$ document source. The Sweave command executes the $R$ code and inserts the results into the relevant place in the document.

To repeat the analysis, the reader can simply re-type all the $\mathrm{R}$ commands shown here. Alternatively, use R CMD Stangle OnlineResourcel. Rnw to extract the R commands from this document into the file Onl ineResourcel. R. Then source ("OnlineResourcel.R") will run all the commands.

\section{Load the code}

This code depends on the spatstat package:

$>$ library (spatstat)

> library (spatstat.utils)

Load the special code:

> source ("usercode.R")

Create a folder to store the generated graphics files (this also checks that we have the necessary file permissions):

> if(!dir.exists("./pix-auto")) dir.create("./pix-auto")

\section{Load data}

\subsection{Synthetic data}

First, here are the synthetic data described in the paper: 


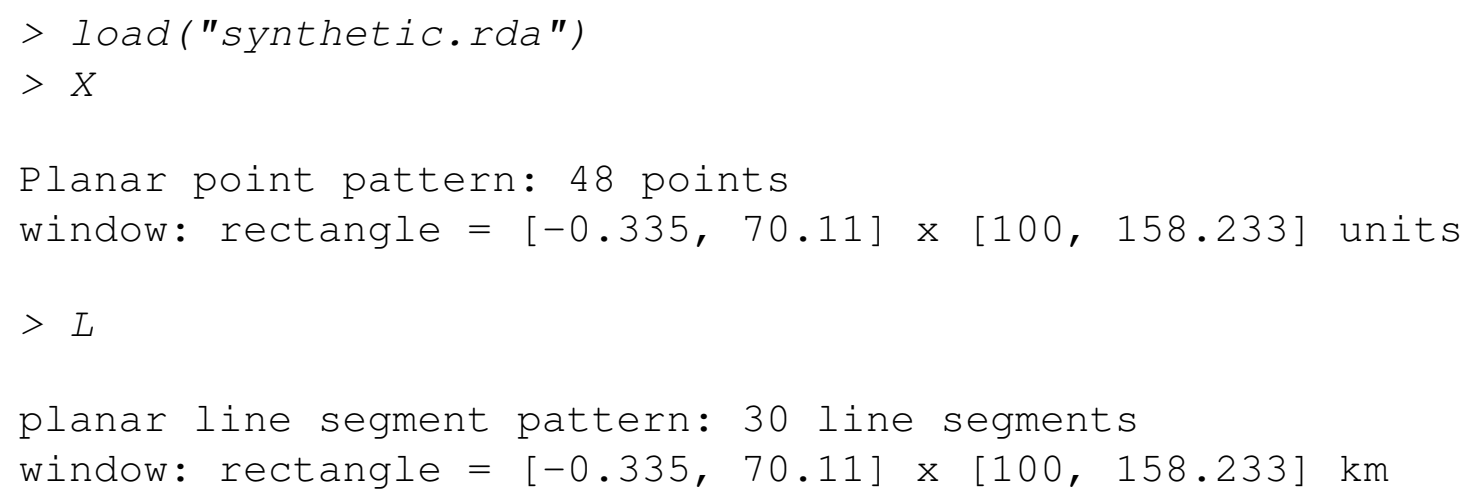

Here $\mathrm{X}$ is the (synthetic) spatial point pattern of deposits, and $\mathrm{L}$ is the spatial pattern of fault lines.

$>\operatorname{plot}(L$, main="synthetic data")

$>\operatorname{plot}(X$, add=TRUE)

\section{synthetic data}

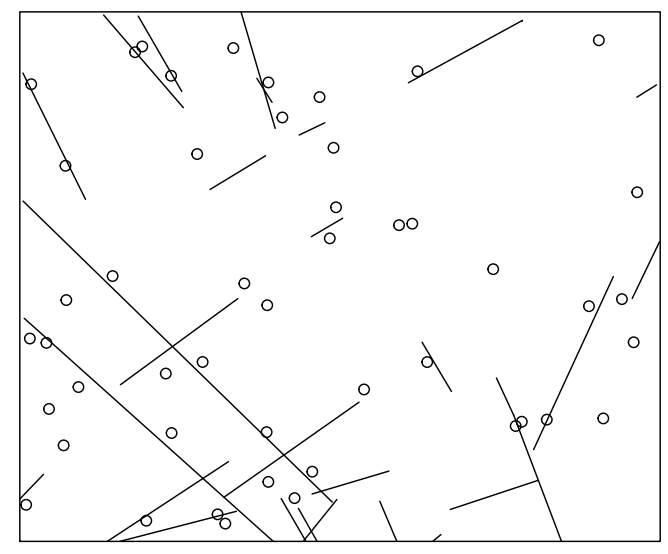

For the covariate $Z$ we will use the distance-to-nearest-fault. This can be computed in the spatstat package by either of the commands distmap or dist fun with slightly different behaviour (documented in the package).

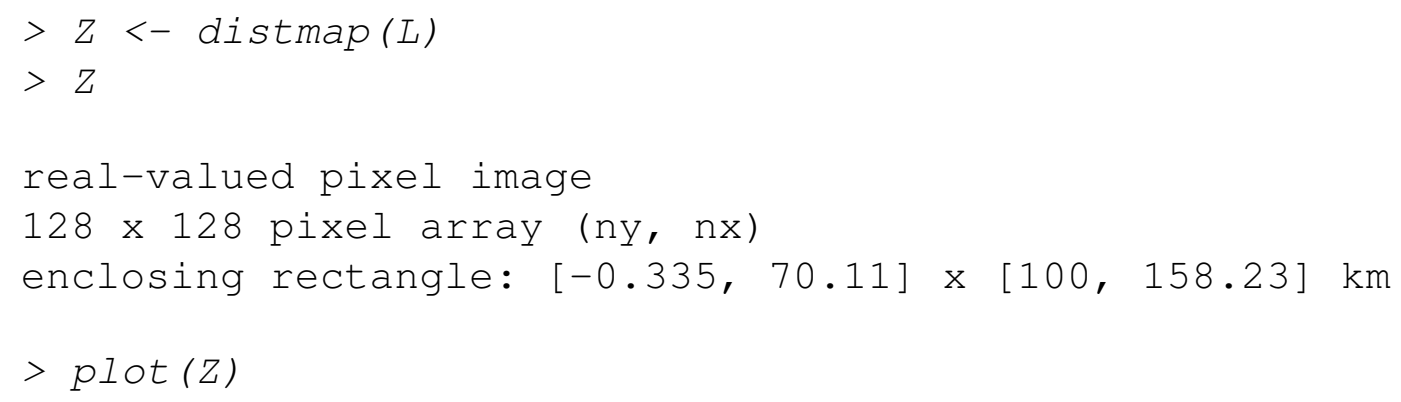




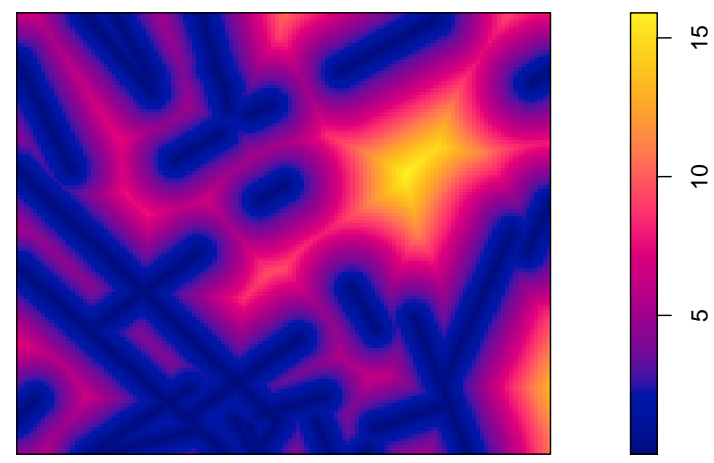

$>$ contour (Z)

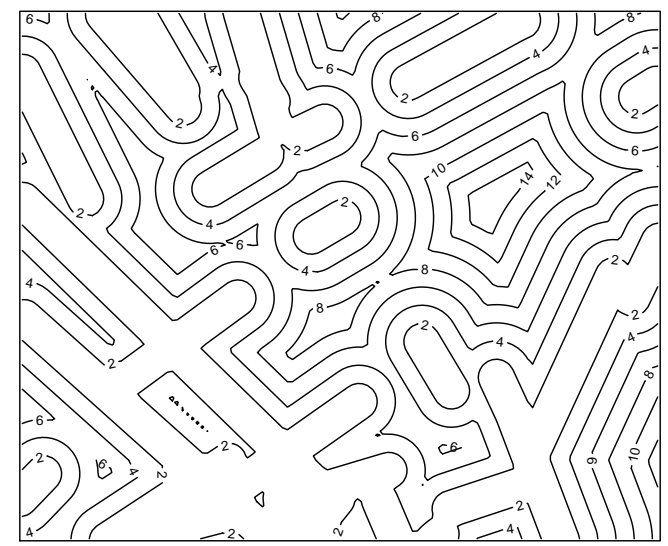

\subsection{Murchison data}

The Murchison gold data are included in the spatstat package.

$>$ murchison

List of spatial objects

gold: 
Planar point pattern: 255 points

window: rectangle $=[352782.9,682589.6] \times[6699742$, 7101484] metres

faults:

planar line segment pattern: 3252 line segments

window: rectangle $=[352782.9,682589.6] \times[6699742$, 7101484] metres

greenstone:

window: polygonal boundary

enclosing rectangle: [352782.9, 681699.6] x [6706467, 7100804] metres

In this case the dataset murchison is a list, containing the different spatial objects (gold deposit pattern, fault pattern, and greenstone feature). The list elements can be extracted individually using $\$$ :

> summary (murchison\$gold)

Planar point pattern: 255 points

Average intensity $1.924569 e-09$ points per square metre

Coordinates are given to 1 decimal place

i.e. rounded to the nearest multiple of 0.1 metres

Window: rectangle $=[352782.9,682589.6] \times[6699742$, 7101484] metres (329800 x 401700 metres)

Window area $=1.32497 e+11$ square metres

Unit of length: 1 metre

Alternatively, they can be accessed using the with operator, which is often neater:

$>$ with(murchison, summary(gold))

Planar point pattern: 255 points

Average intensity 1.924569 e-09 points per square metre

Coordinates are given to 1 decimal place

i.e. rounded to the nearest multiple of 0.1 metres

Window: rectangle $=[352782.9,682589.6] \times[6699742$, 7101484] metres (329800 x 401700 metres)

Window area $=1.32497 e+11$ square metres

Unit of length: 1 metre

Let's rescale all the Murchison data from metres to kilometres:

$>$ mur <- solapply (murchison, rescale, s=1000, unitname="km")

and add the distance-to-nearest-fault covariate: 
$>$ mur\$dfault <- distfun(mur\$faults)

The new list mur contains the rescaled Murchison data and the derived covariate.

$>$ plot (mur\$faults, main="Murchison data")

> plot (mur\$gold, add=TRUE, pch=3, col="blue")

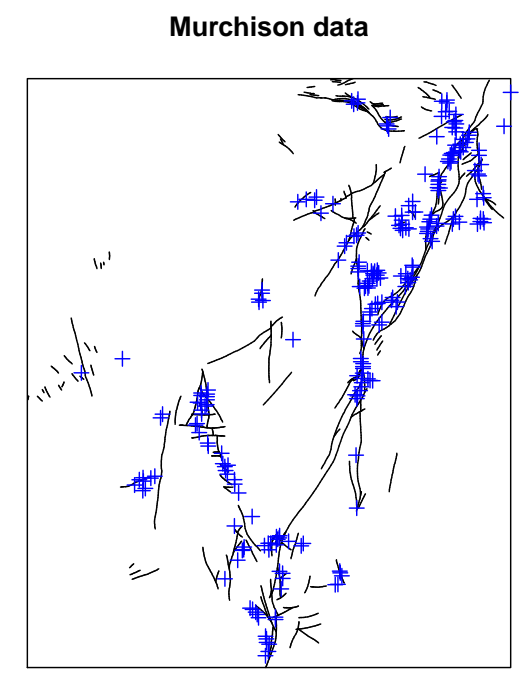

\section{Threshold selection}

The command select Thresh defined in usercode. R selects the threshold automatically. The user can specify the method.

For the synthetic data:

$>\operatorname{selectThresh}(X, Z$, method=" $t ")$

Z

1.424575

$>\operatorname{selectThresh}(X, Z$, method="Y")

Z

1.966457

The first result indicates that the threshold obtained by maximising the Studentised contrast $t$ was $1.425 \mathrm{~km}$. The second result indicates that the threshold obtained by maximising the Youden criterion was $1.966 \mathrm{~km}$.

The result of selectThresh can also be plotted to show the criterion as a function of the threshold value: 
$>b<-$ selectThresh( $X, Z$, method="Y")

$>$ plot(b, main = "Simulated data, Youden criterion",

$+\quad y l a b=$ "Youden criterion $Y(z) "$ ")

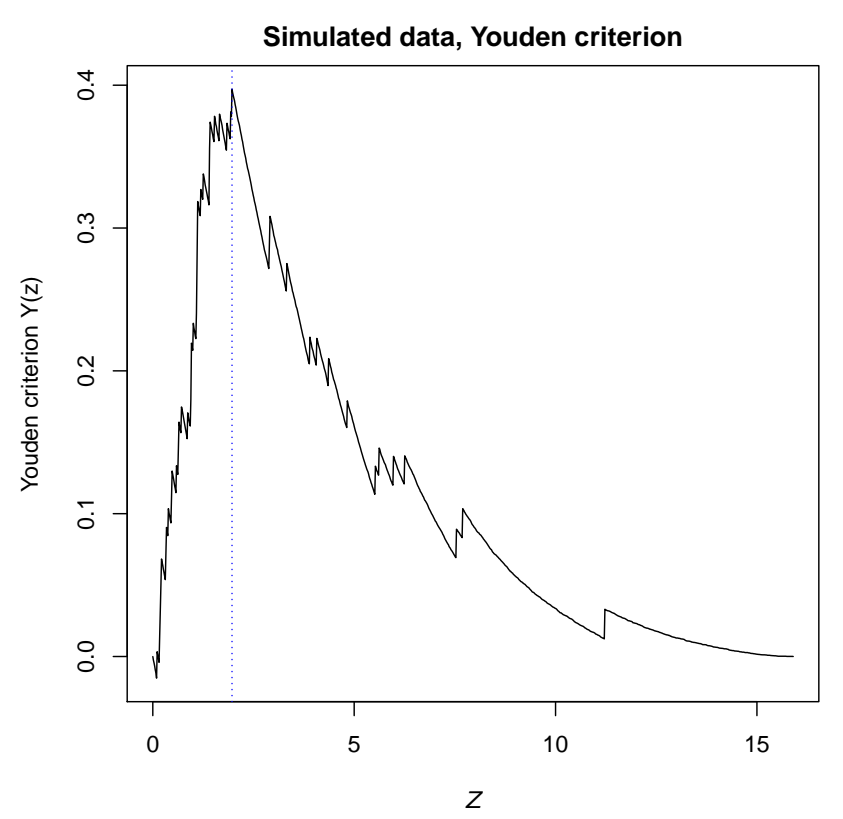

For the Murchison data:

$>b<-$ with(mur, selectThresh(gold, dfault, method="Y"))

$>b$

dfault

6.655075

$>$ plot(b, main="Murchison data, Youden criterion",

$+\quad y l a b=$ "Youden criterion $Y(z) "$ )

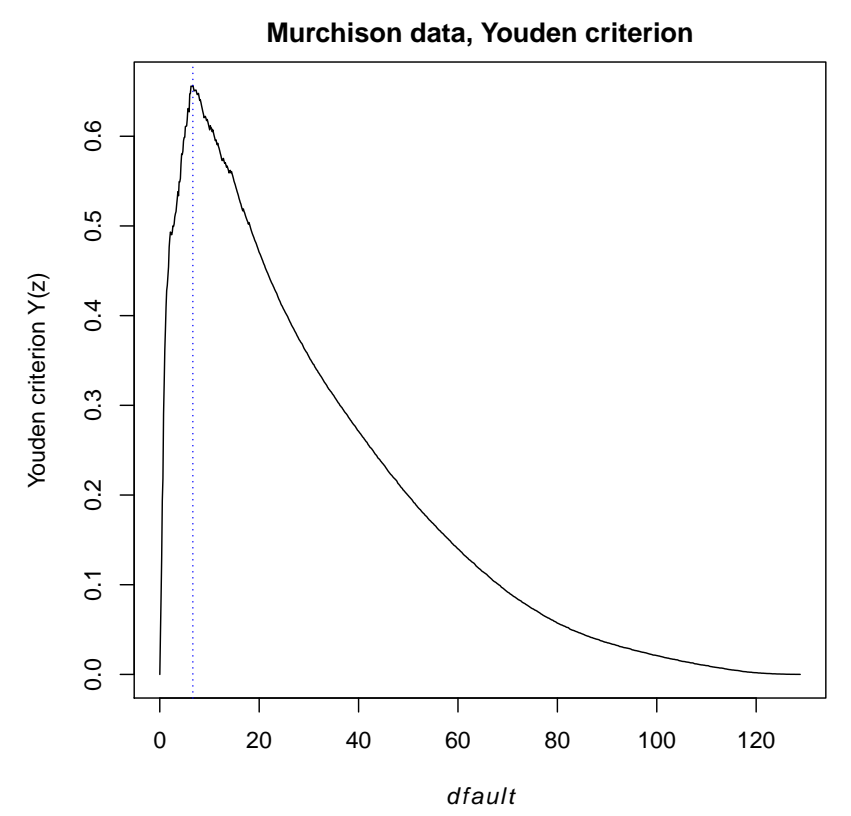




\section{Bootstrap confidence intervals}

The functions boot SE and bootCI defined in usercode. R compute (respectively) bootstrap standard errors and bootstrap confidence intervals for the threshold, using the Youden criterion. The usage of the two functions is very similar, so we only show the computation for confidence intervals.

\subsection{Synthetic data}

For the synthetic data:

$>\operatorname{bootCI}(X, Z)$

$\begin{array}{rrrr} & 10 & \text { hi } & \text { conf } \\ \text { z } & 1.2038740 & 2.2558028 & 0.949 \\ \text { s } & 0.1956055 & 0.3521729 & 0.949\end{array}$

The first row shows the nonparametric bootstrap confidence interval for the threshold distance $z$ in kilometres (true value $2 \mathrm{~km}$ ). The second row shows the corresponding confidence interval for the fraction of area $s$.

The parametric bootstrap confidence interval is:

$>\operatorname{bootCI}(X, Z$, parametriC=TRUE)

$\begin{array}{rrrr} & \text { lo } & \text { hi } & \text { conf } \\ \text { z } & 1.592587 & 2.6448494 & 0.95 \\ \text { s } & 0.256665 & 0.4057007 & 0.95\end{array}$

In the section Bootstrap standard errors and confidence intervals of the main paper, we suggested that the parametric bootstrap is typically more accurate, provided the assumed model (for the simulated data, the simple threshold model) is true. Indeed, we see this to be the case here as the nonparametric bootstrap distribution is bimodal, but the parametric distribution is unimodal and asymmetric, as was also suggested in the main paper.

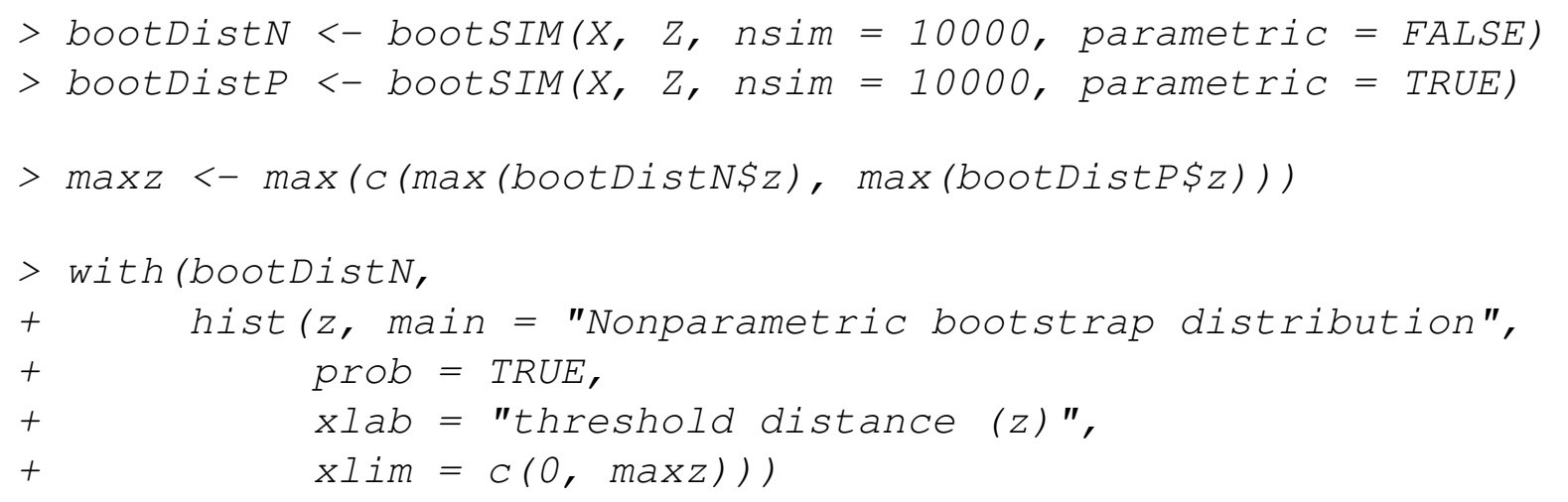



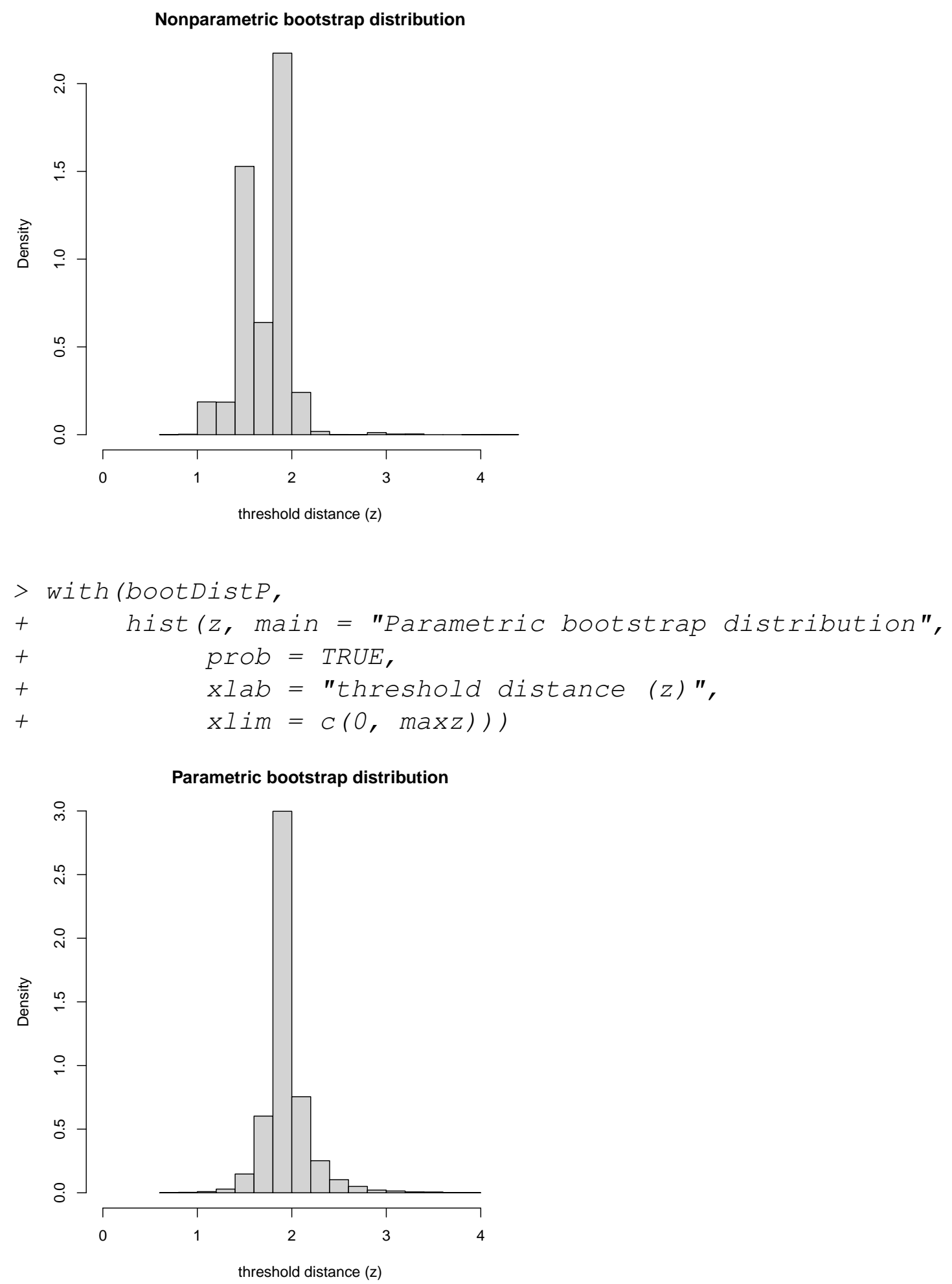

\subsection{Murchison gold data}

For the Murchison gold data:

$>$ with(mur, bootCI(gold, dfault)) 


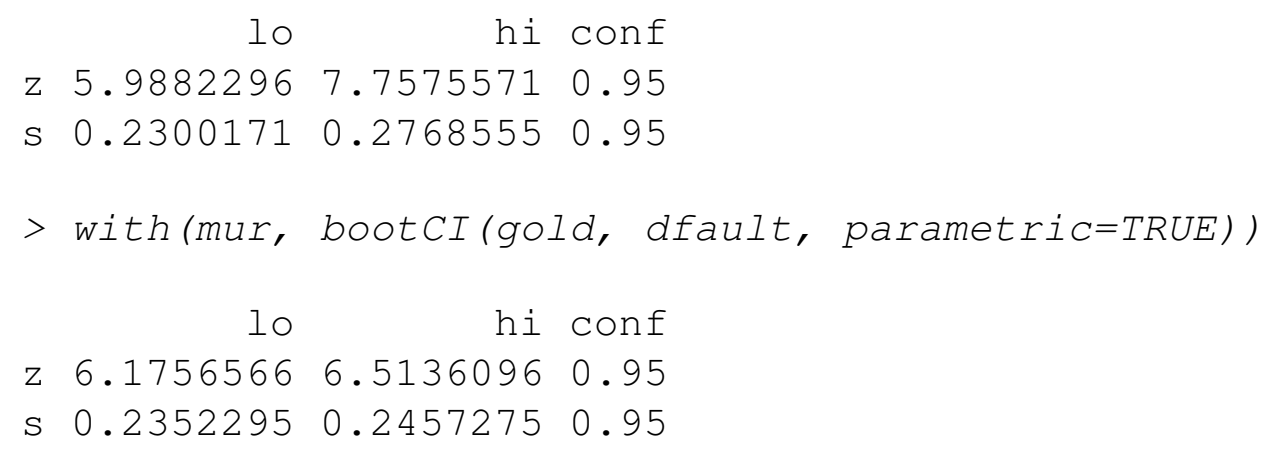

The parametric bootstrap confidence interval (obtained with the option parametric=TRUE) is not reliable for the Murchison data because they do not seem to conform to the simple threshold model. As pointed out in the main paper, the nonparametric bootstrap is more robust against violation of the model assumptions, and we see below that that the nonparametric bootstrap distribution is both unimodal and asymmetric.
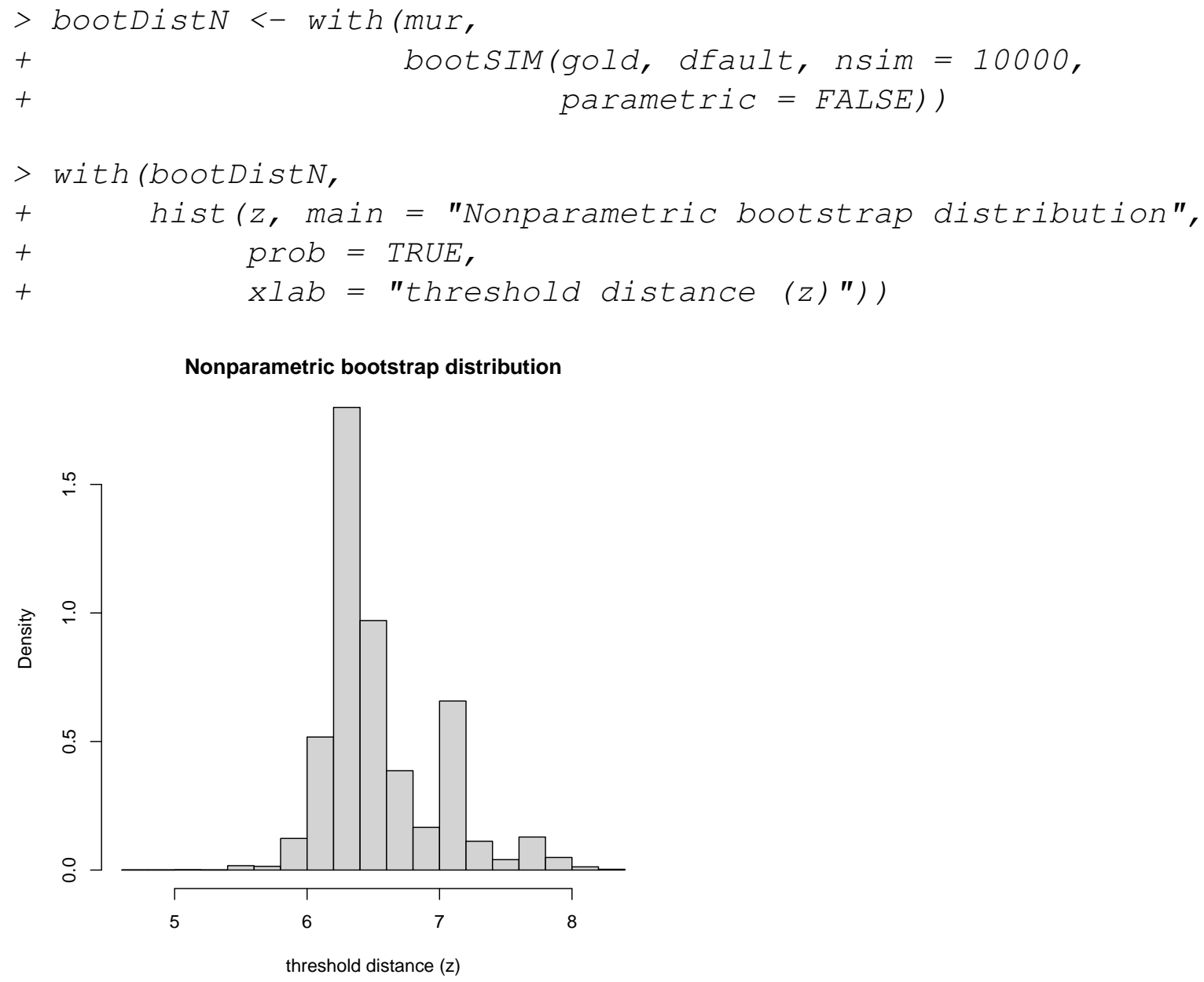


\title{
Online Resource 2
}

\author{
Online supplement to \\ Optimal thresholding of predictors in mineral prospectivity analysis \\ by Baddeley et al. (2020)
}

The file OnlineResource1.pdf demonstrates how to calculate the threshold of the distance-tonearest-fault for the Murchison data and for synthetic data. In addition, the code calculates bootstrap confidence intervals for the threshold based on the Youden criterion.

In this document, we show the results of carrying out the same analysis but with additional realizations of the synthetic data. Using a fixed pattern of fault lines and fixed threshold distance, additional spatial point patterns of deposits have been generated using the same simple threshold model used to generate the synthetic data in Figure 5 of the main paper. The results below confirm that when the simple threshold model applies, the parametric bootstrap yields more accurate and sensible confidence intervals for the threshold distance. In addition, the following discussion outlines the practical value of examining different realizations of synthetic data.

Geologists have only a single pattern of deposit points to estimate a threshold and classify an area into prospective and non-prospective zones. Although geological features like faults, rock unit boundaries and areas of favourable host rock determine the sites of gold deposition, the threshold estimate is very dependent on the point pattern of the known deposits. It is important to recognize that this structural framework of rock units, and geometry of faults and shear zones controls the potential location of gold deposits and therefore the prospectivity. But our knowledge of the deposits and occurrences which provide evidence for this prospectivity is incomplete and the precise location of deposits at the scale of minor faults (a few kilometres or less) that they are associated with is random. There are two further aspects to the randomness of the deposit pattern:

1. As additional exploration is carried out, new mineral occurrences and deposits are often discovered so with time additional points are added to the pattern.

2. Generally, only gold deposits located within $300 \mathrm{~m}$ of the surface are discoverable by drilling and geochemical exploration, yet orogenic gold deposits were originally formed at depths from 5 to at least $15 \mathrm{~km}$. There is no reason to consider that the present-day surface zone is more or less prospective, in terms of deposit numbers or gold content, than any other slice through the upper crust. A complex network of faults and shear zones together with differential uplift and erosion determined which depths at the time of gold mineralization are exposed at the surface today. Consequently, the pattern of deposits we have discovered or can potentially discover is the result of random processes within areas where the prospectivity is determined by a larger scale framework of geodynamic events and processes and structural features.

Given that the pattern of deposits we observe is only one of many possible patterns, it makes sense to examine the effect of alternative sets of random locations of deposits within the favourable zones on our estimates of threshold distance. It should be noted though, that although the location of the deposit points change in the simulations, the numbers and densities of deposits in the prospective zones that define the optimal threshold remain the same as those described for the synthetic dataset in the main text. 


\section{Original synthetic data}

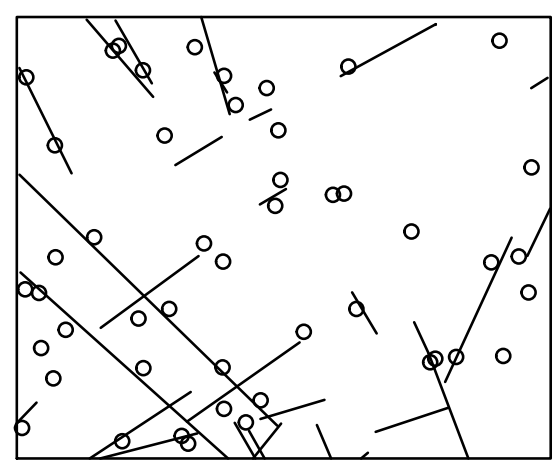

Figure 1: Faults shown as solid lines, deposits as open circles.

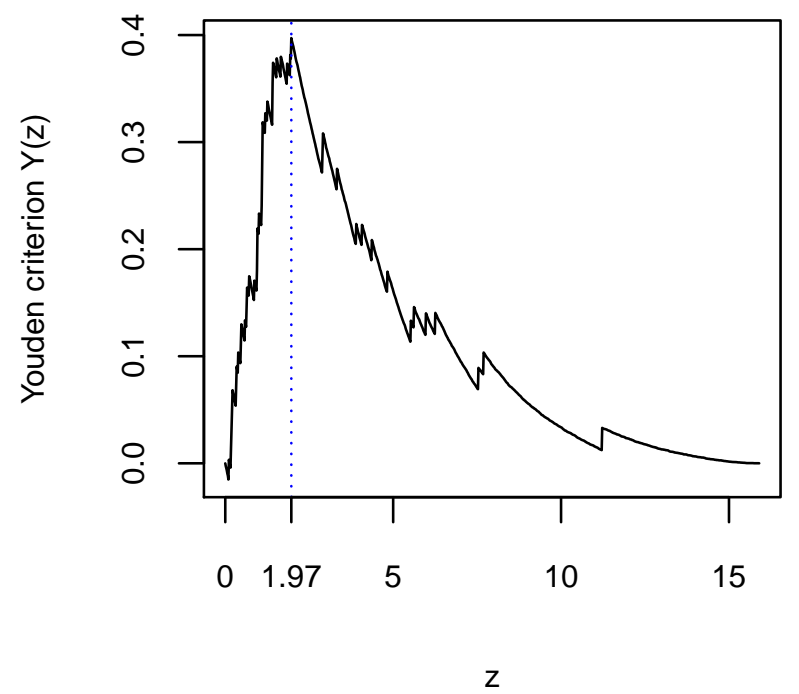

Figure 2: Youden criterion, showing the estimate of the threshold

For the nonparametric and parametric bootstrap, 95\% confidence intervals are shown followed by the corresponding bootstrap distributions.

Table 1: 95\% nonparametric bootstrap confidence interval for the threshold estimate $(z)$ and fraction of area (s).

\begin{tabular}{rrrr}
\hline & lo & hi & conf \\
\hline $\mathrm{z}$ & 1.24 & 2.93 & 0.95 \\
$\mathrm{~s}$ & 0.20 & 0.44 & 0.95 \\
\hline
\end{tabular}

Table 2: 95\% parametric bootstrap confidence interval for the threshold estimate $(\mathrm{z})$ and fraction of area (s).

\begin{tabular}{rrrr}
\hline & lo & hi & conf \\
\hline z & 1.59 & 2.50 & 0.95 \\
$\mathrm{~s}$ & 0.26 & 0.39 & 0.95 \\
\hline
\end{tabular}


Nonparametric bootstrap distribution

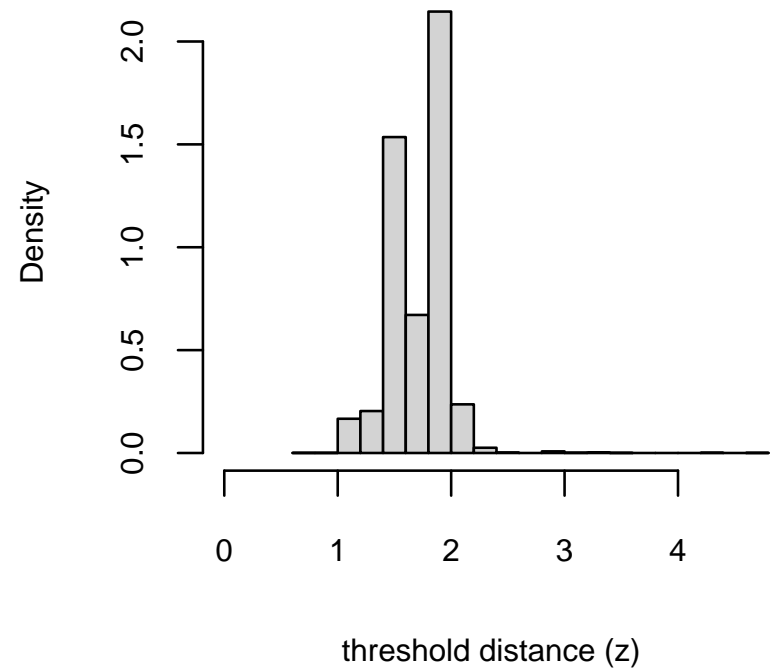

Figure 3: Nonparametric bootstrap distribution of the threshold distance selected by the Youden criterion.

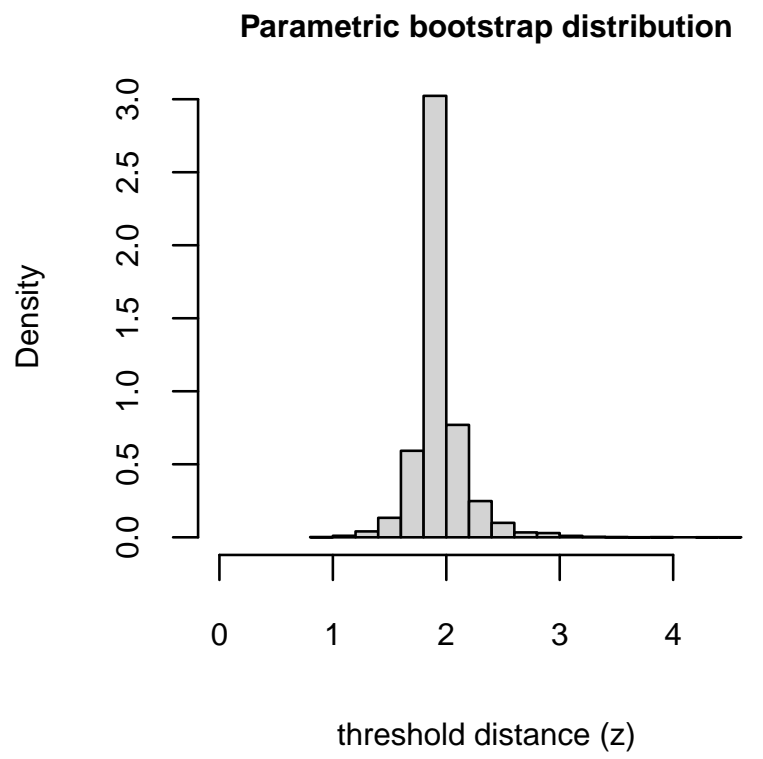

Figure 4: Parametric bootstrap distribution of the threshold distance selected by the Youden criterion. 


\section{Realization 2}

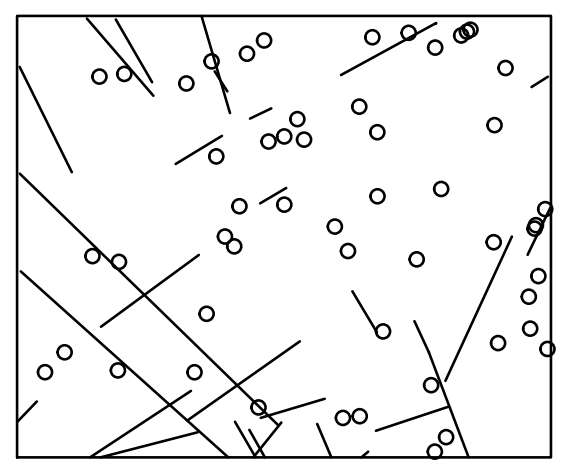

Figure 5: Faults shown as solid lines, deposits as open circles.

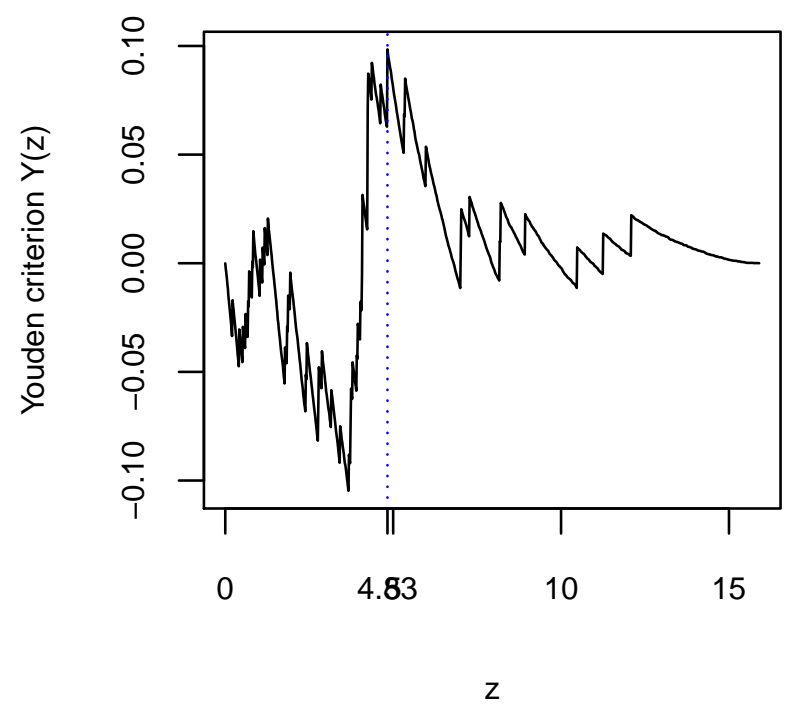

Figure 6: Youden criterion, showing the estimate of the threshold

For the nonparametric and parametric bootstrap, 95\% confidence intervals are shown followed by the corresponding bootstrap distributions.

Table 3: 95\% nonparametric bootstrap confidence interval for the threshold estimate $(\mathrm{z})$ and fraction of area (s).

\begin{tabular}{rrrr}
\hline & lo & hi & conf \\
\hline $\mathrm{z}$ & 0.89 & 10.92 & 0.95 \\
$\mathrm{~s}$ & 0.15 & 0.96 & 0.95 \\
\hline
\end{tabular}

Table 4: 95\% parametric bootstrap confidence interval for the threshold estimate $(\mathrm{z})$ and fraction of area (s).

\begin{tabular}{rrrr}
\hline & lo & hi & conf \\
\hline $\mathrm{z}$ & 1.14 & 7.75 & 0.95 \\
$\mathrm{~s}$ & 0.19 & 0.88 & 0.95 \\
\hline
\end{tabular}




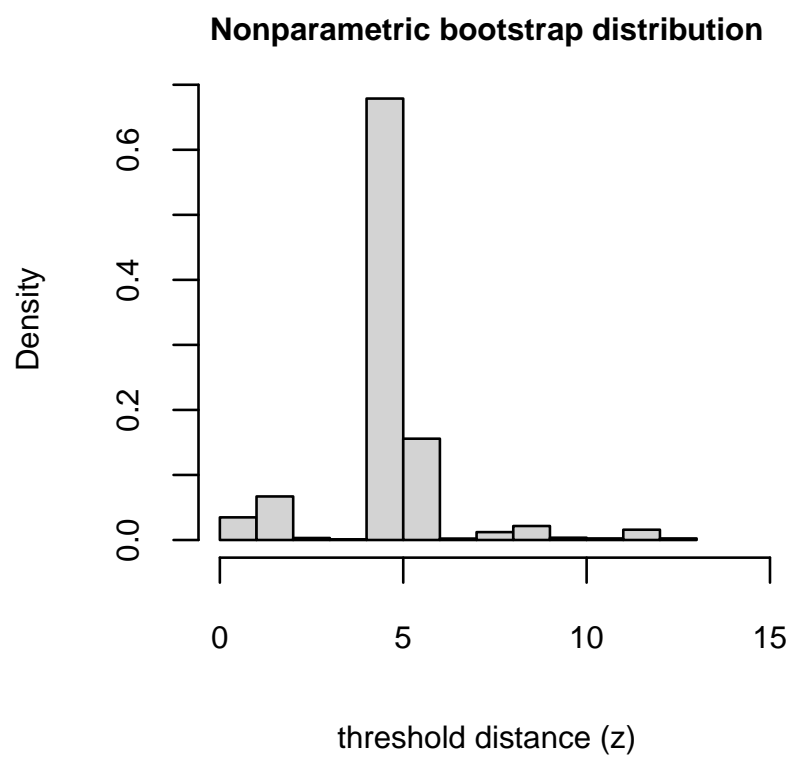

Figure 7: Nonparametric bootstrap distribution of the threshold distance selected by the Youden criterion.

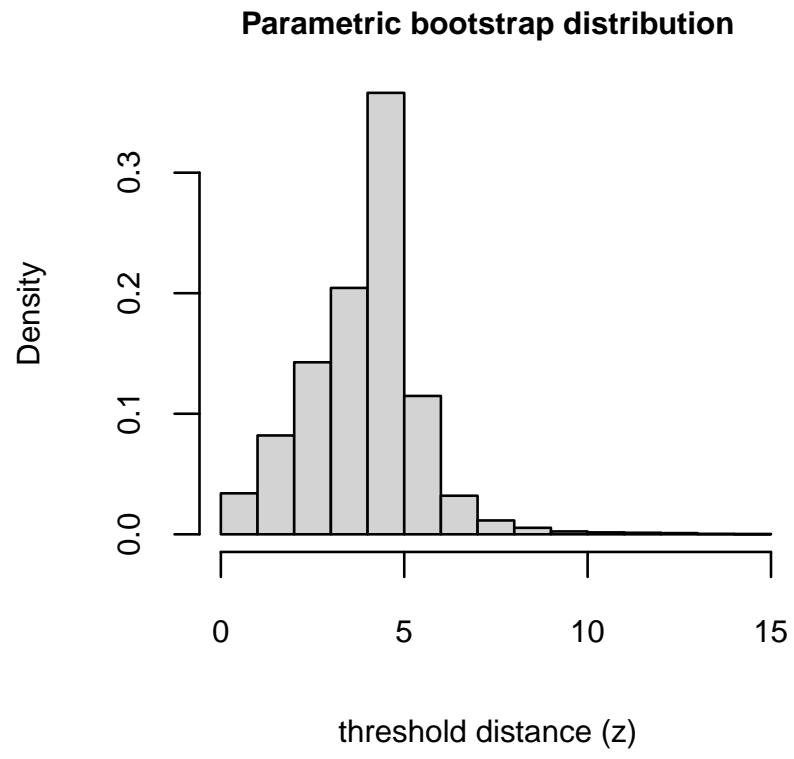

Figure 8: Parametric bootstrap distribution of the threshold distance selected by the Youden criterion. 


\section{Realization 3}

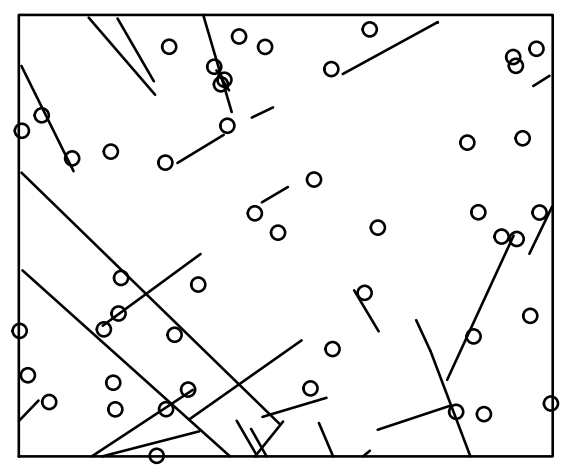

Figure 9: Faults shown as solid lines, deposits as open circles.

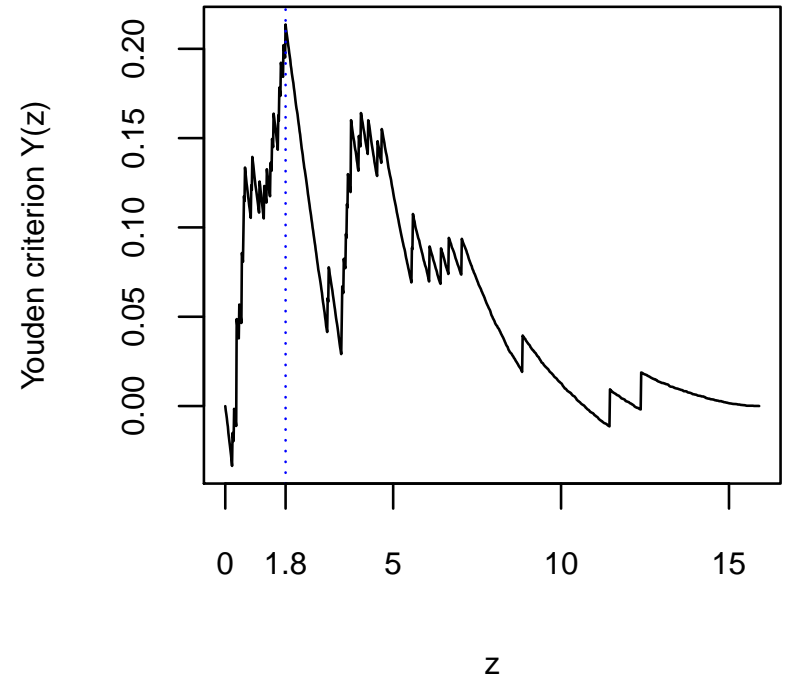

Figure 10: Youden criterion, showing the estimate of the threshold

For the nonparametric and parametric bootstrap, 95\% confidence intervals are shown followed by the corresponding bootstrap distributions.

Table 5: 95\% nonparametric bootstrap confidence interval for the threshold estimate (z) and fraction of area (s).

\begin{tabular}{rrrr}
\hline & lo & hi & conf \\
\hline z & 0.6 & 5.18 & 0.95 \\
$\mathrm{~s}$ & 0.1 & 0.69 & 0.95 \\
\hline
\end{tabular}

Table 6: 95\% parametric bootstrap confidence interval for the threshold estimate $(\mathrm{z})$ and fraction of area (s).

\begin{tabular}{rrrr}
\hline & lo & hi & conf \\
\hline $\mathrm{z}$ & 1.06 & 3.86 & 0.95 \\
$\mathrm{~s}$ & 0.17 & 0.56 & 0.95 \\
\hline
\end{tabular}




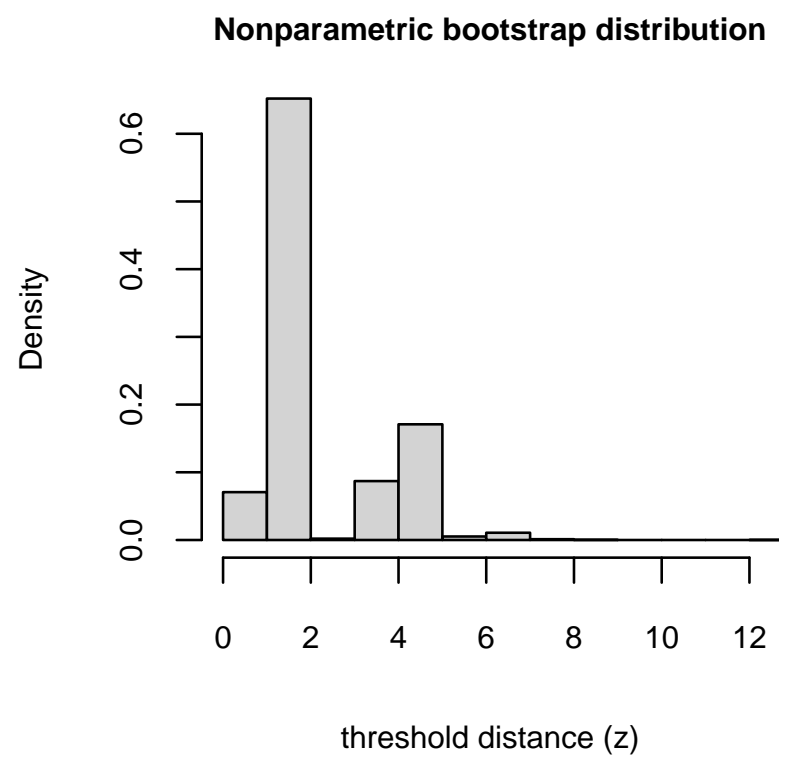

Figure 11: Nonparametric bootstrap distribution of the threshold distance selected by the Youden criterion.

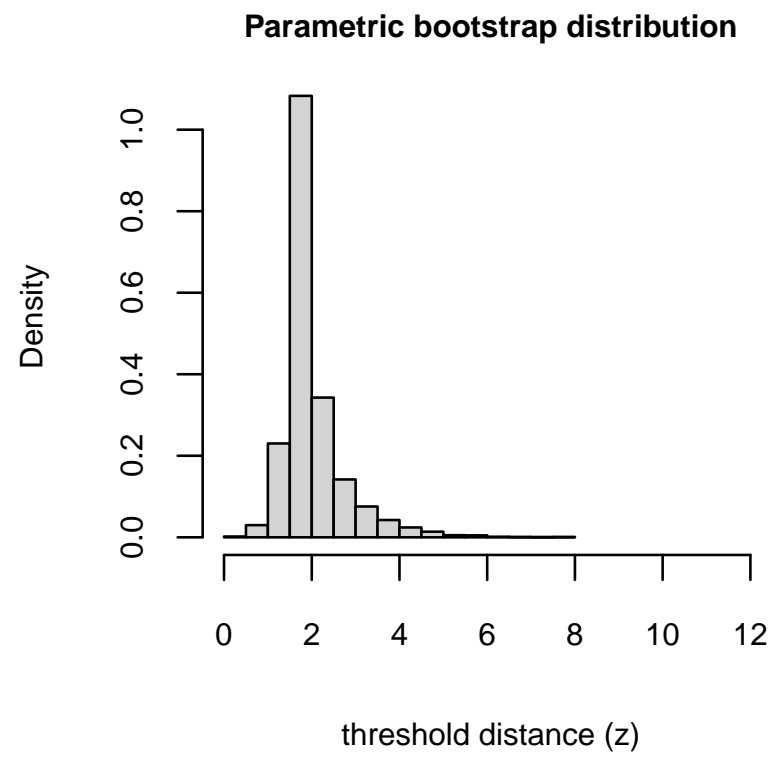

Figure 12: Parametric bootstrap distribution of the threshold distance selected by the Youden criterion. 


\section{Realization 4}

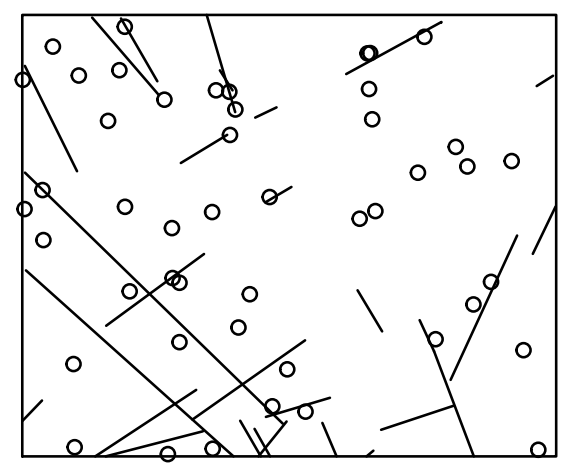

Figure 13: Faults shown as solid lines, deposits as open circles.

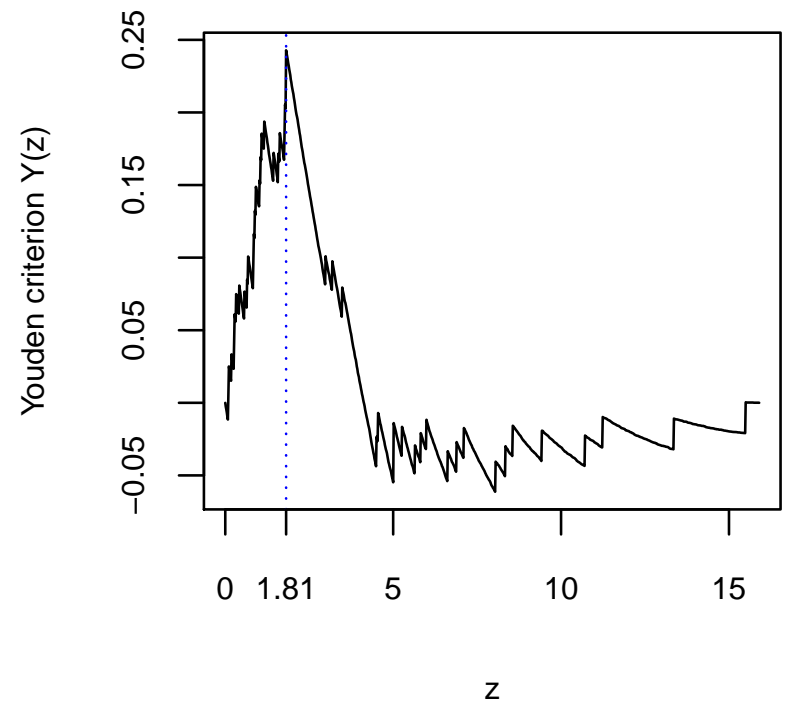

Figure 14: Youden criterion, showing the estimate of the threshold

For the nonparametric and parametric bootstrap, 95\% confidence intervals are shown followed by the corresponding bootstrap distributions.

Table 7: 95\% nonparametric bootstrap confidence interval for the threshold estimate $(z)$ and fraction of area (s).

\begin{tabular}{rrrr}
\hline & lo & hi & conf \\
\hline $\mathrm{z}$ & 0.91 & 1.92 & 0.95 \\
$\mathrm{~s}$ & 0.15 & 0.30 & 0.94 \\
\hline
\end{tabular}

Table 8: 95\% parametric bootstrap confidence interval for the threshold estimate (z) and fraction of area (s).

\begin{tabular}{rrrr}
\hline & lo & hi & conf \\
\hline $\mathrm{z}$ & 1.09 & 3.57 & 0.95 \\
$\mathrm{~s}$ & 0.18 & 0.52 & 0.95 \\
\hline
\end{tabular}




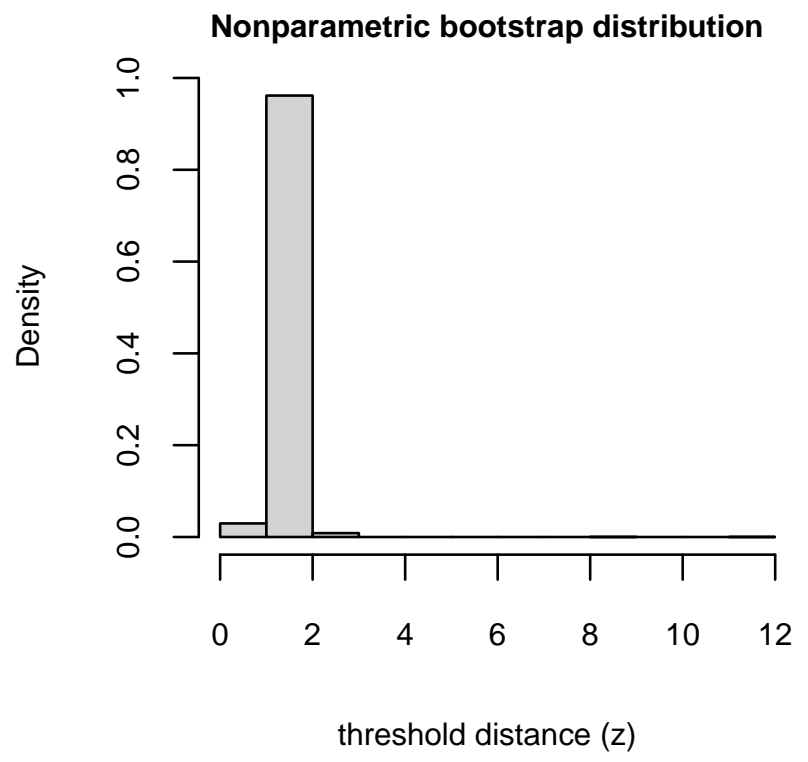

Figure 15: Nonparametric bootstrap distribution of the threshold distance selected by the Youden criterion.

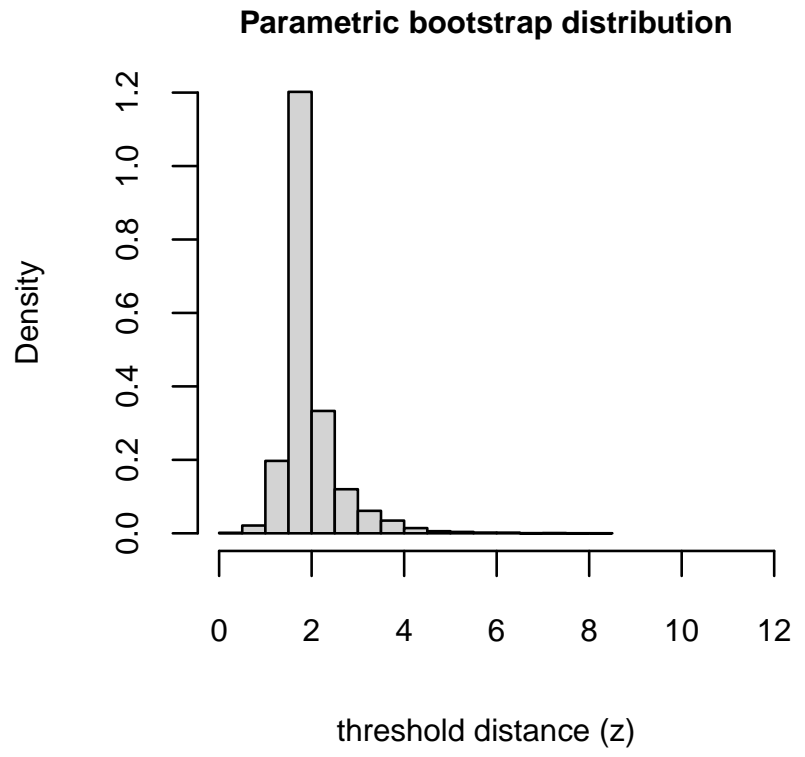

Figure 16: Parametric bootstrap distribution of the threshold distance selected by the Youden criterion. 